University of Louisville ThinkIR: The University of Louisville's Institutional Repository

Electronic Theses and Dissertations

$8-2014$

\title{
Taking clinical judgment out of the equation : a call for the standardization of MCI diagnostic criteria and construction of a model to predict conversion to dementia.
}

Adam Tyler Gerstenecker

University of Louisville

Follow this and additional works at: https://ir.library.louisville.edu/etd

Part of the Psychology Commons

\section{Recommended Citation}

Gerstenecker, Adam Tyler, "Taking clinical judgment out of the equation : a call for the standardization of MCI diagnostic criteria and construction of a model to predict conversion to dementia." (2014). Electronic Theses and Dissertations. Paper 490.

https://doi.org/10.18297/etd/490

This Doctoral Dissertation is brought to you for free and open access by ThinkIR: The University of Louisville's Institutional Repository. It has been accepted for inclusion in Electronic Theses and Dissertations by an authorized administrator of ThinkIR: The University of Louisville's Institutional Repository. This title appears here courtesy of the author, who has retained all other copyrights. For more information, please contact thinkir@louisville.edu. 
TAKING CLINICAL JUDGMENT OUT OF THE EQUATION: A CALL FOR THE STANDARDIZATION OF MCI DIAGNOSTIC CRITERIA AND CONSTRUCTION

OF A MODEL TO PREDICT CONVERSION TO DEMENTIA

\author{
By \\ Adam Tyler Gerstenecker \\ B.S., Southern Illinois University at Carbondale, 2001 \\ M.S., Murray State University, 2007

\begin{abstract}
A Dissertation
Submitted to the Faculty of the

College of Arts and Sciences of the University of Louisville

In Partial Fulfillment of the Requirements

For the Degree of
\end{abstract}

Doctor of Philosophy

Department of Psychological and Brain Sciences

University of Louisville

Louisville, Kentucky

August 2014 
Copyright 2012 by Adam Tyler Gerstenecker

All rights reserved 

TAKING CLINICAL JUDGMENT OUT OF THE EQUATION: A CALL FOR THE STANDARDIZATION OF MCI DIAGNOSTIC CRITERIA AND CONSTRUCTION OF A MODEL TO PREDICT CONVERSION TO DEMENTIA

$$
\text { By }
$$

Adam Gerstenecker

B.S., Southern Illinois University at Carbondale, 2001

M.S., Murray State University, 2007

Dissertation Approved on

June 26, 2014

By the following Dissertation Committee:

Benjamin Mast, $\mathrm{PhD}$

Suzanne Meeks, $\mathrm{PhD}$

Janet Woodruff-Borden, $\mathrm{PhD}$

Robert Friedland, MD 


\begin{abstract}
TAKING CLINICAL JUDGMENT OUT OF THE EQUATION: A CALL FOR THE STANDARDIZATION OF MCI DIAGNOSTIC CRITERIA AND CONSTRUCTION OF A MODEL TO PREDICT CONVERSION TO DEMENTIA
\end{abstract}

Adam Gerstenecker

June 26, 2014

\begin{abstract}
Although the diagnostic criteria for mild cognitive impairment have evolved considerably since their inception, they remain varied and able to be interpreted and implemented in different ways depending on the judgment of the clinician. Because of this issue, a wide range of incidence, prevalence, and conversion rates are found in the research literature. Using data collected from 400 patients with amnestic mild cognitive impairment, limitations inherent in current mild cognitive impairment diagnostic criteria were addressed. First, using Alzheimer's Disease Neuroimaging Initiative diagnostic criteria, an equation was constructed to predict conversion from mild cognitive impairment to dementia by analyzing the predictive ability of variables representative of a number of categories (i.e., demographic, psychiatric, functional, biomarker, imaging, and cognitive). This model accounted for over $60 \%$ of variance in conversion and exhibited an area under the curve of 0.93 . Then, separate models were constructed using different applications of the current diagnostic criteria for mild cognitive impairment. As expected, criteria utilizing a one standard deviation clinical cutoff on a measure of delayed verbal recall in combination with the allowance for some functional change (i.e.,
\end{abstract}


scores $\leq 5$ on the Functional Activities Questionnaire) exhibited the greatest utility of any combination of diagnostic criteria. Taken together, these results indicate that statistical equations can be constructed to predict conversion from mild cognitive impairment to dementia and be tailored for widespread clinical use. Moreover, these results show that the standardization of current diagnostic criteria for mild cognitive impairment is clearly needed. 


\section{TABLE OF CONTENTS}

PAGE

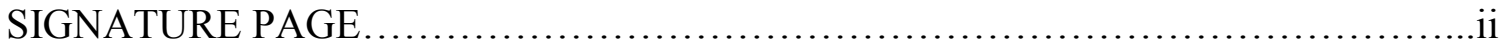

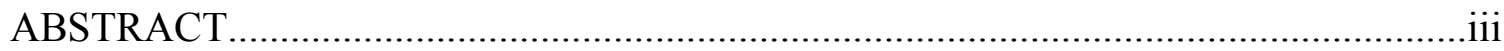

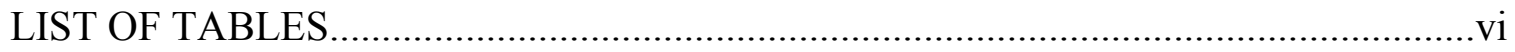

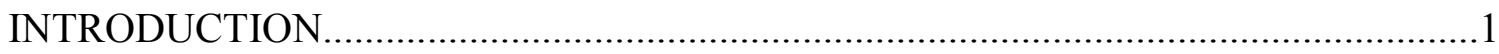

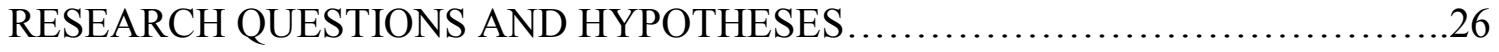

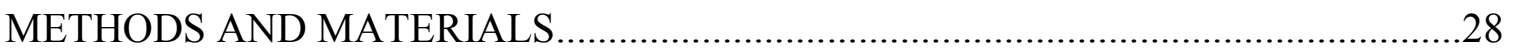

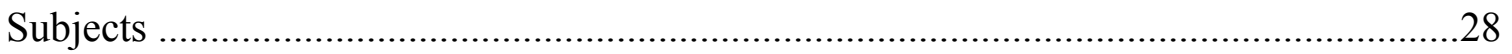

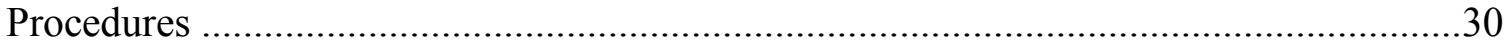

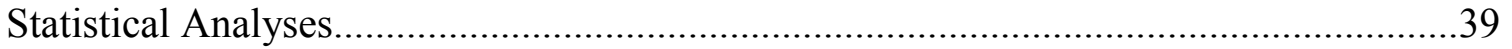

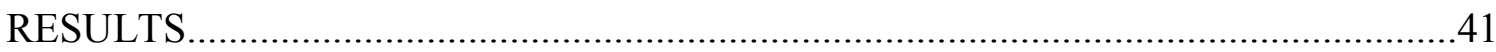

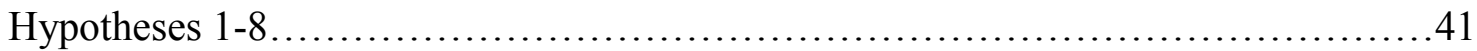

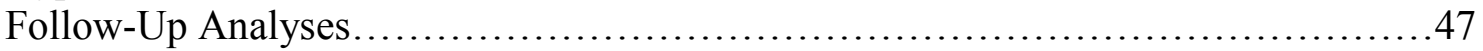

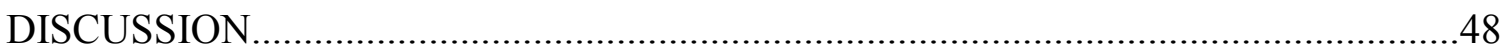

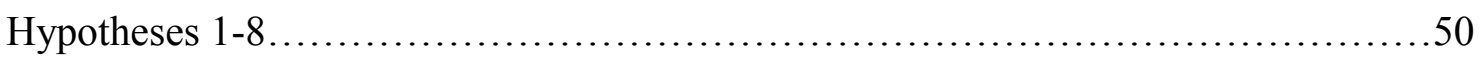

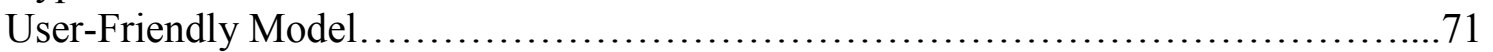

Limitations and Future Directions.............................................. 72

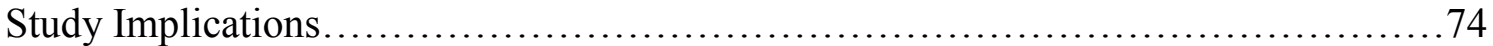

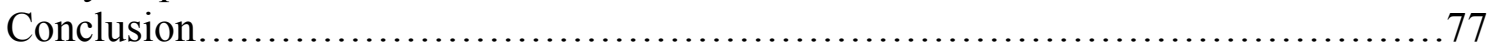

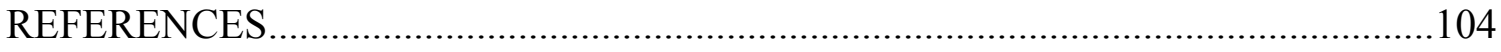

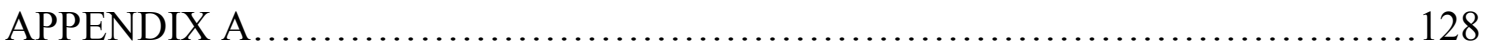

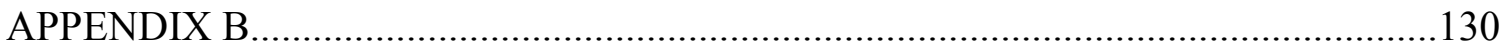

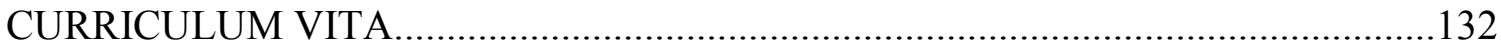




\section{LIST OF TABLES}

TABLE

PAGE

1. MCI Diagnostic Criteria and Current Recommendations..........................

2. MCI-R Subtype and Etiology

3. Recommendations from the Alzheimer's Group..............................................44

4. Biomarkers Under Examination for AD.....................................69

5. MCI Criteria Incorporating Biomarkers.................................8 87

6. Current Recommendations for Standardization of MCI Diagnostic Criteria

7. Diagnostic Criteria for Classification Systems.

8. Demographics for MCI Patients by Classification System.

9. Neurocognitive Model using ADNI Backward Elimination Method

10. Imaging Model using ADNI Backward Elimination Method

11. Final Model using ADNI Criteria, Hand Selected Method

12. Number and Percentage of Patients Identified as Normal, MCI, and Mild AD and

1 Converting from MCI to dementia.

13. Final Model using ADNI Criteria, Backward Elimination Method

14. Final Model using Proposed Criteria \#1(Model \#1).

15. Final Model using Proposed Criteria \#2 (Model \#2).

16. Final Model using Proposed Criteria \#3 (Model \#3).

17. Performance of Models across Classification System $(\beta, p)$.

18. Performance of Models across Classification Systems (Pseudo R ${ }^{2}, A U C$ ).

19. User-Friendly Model 


\section{INTRODUCTION}

\section{Mary and the Diagnostic Process}

Mary is a 67-year-old Caucasian woman whose family is concerned that she is "having trouble with her memory." Her daughter reports that Mary exhibits some difficulty "keeping up" in conversations and recently "lost track of her hand" while playing bridge. This was noted as especially distressing to Mary's daughter because Mary has been an avid bridge player for over 40 years and "quite the card shark." In contrast to his sister, Mary's son reports that his mother's memory is "no different from other people [her] age." When Mary is asked about her memory, she reports "it is just fine." Mary's primary concern is "feeling depressed" and "not being able to stay asleep at night because [she] worries too much."

After completing a neuropsychological battery, Mary exhibited average performance when compared to age- and education-matched peers on a test of general cognitive functioning $\left(25^{\text {th }}\right.$ percentile). No subscale scores fell more than 1.5 standard deviations (SD) below the mean. However, on a more comprehensive test of memory, Mary exhibited below average immediate $\left(20^{\text {th }}\right.$ percentile $)$ and delayed recall $\left(15^{\text {th }}\right.$ percentile). Mary's family reports that she is completely independent in both higher- and lower-order activities of daily living (ADL), but Mary was not administered a functional measure as part of her neuropsychological evaluation. Given these observations, her attending physician recommended she be diagnosed with mild cognitive impairment 
(MCI) and prescribed Aricept (a medication designed to target memory function in older adults). However, the neuropsychologist who administered the tests recommended she be re-evaluated in 6-9 months after being treated for depression and anxiety.

Nevertheless, Mary was diagnosed with MCI, the diagnosis recorded in her medical records, and her family educated about the diagnosis and increased risk MCI patients have for progression to a dementia syndrome.

\section{Study Purpose}

The purpose of the current study is to demonstrate how MCI diagnostic criteria are affected by a lack of standardization and clinical judgment. This issue will be discussed in detail, and a potential solution proposed. This solution will take the form of two statistical models: (1) a "screening" model designed to increase the standardization of diagnostic criteria, and (2) a "conversion" model designed to fully remove clinical judgment from the diagnostic process. The screening model will primarily be based upon the most widely used and accepted MCI clinical diagnostic criteria. However, particular emphasis will be given to decreasing the ambiguity of these criteria so they can be applied in a consistent manner. For the conversion model, data from a full clinical and medical evaluation will be used to construct a mathematical equation designed to identify people at particular risk of converting from MCI to dementia. The proposed conversion model will be based upon the combined predictive power of numerous factors including neuropsychological performance, neuroimaging, biomarkers, behavioral abnormalities, and functional ability. 


\section{Mary's Diagnosis and Clinical Judgment}

Mary's case illustrates the prominent role clinical judgment assumes in the evaluation of older adults with suspected cognitive impairment. But, how can clinical judgment play such a prominent role in a process so seemingly driven by data? The answer is simple: statistical models of diagnostic prediction are not readily available to professionals nor used by professionals when working with older adults. Consequently, these professionals often rely on heuristics. In short, heuristics are mental tools based on prior experience and knowledge that are used to reduce cognitive burden in decision making and to allow for faster and often correct decisions (Shah \& Oppenheimer, 2008). However, by introducing personal heuristics into the decision-making process, personal cognitive biases are also introduced and contribute to errors in judgment (Tversky \& Kahneman). Consequently, two clincians with the best interests of a patient in mind could have quite different diagnostic impressions of the very same set of presenting symptoms.

For example, Mary's physician frequently encounters patients whose families report memory loss. More often than not, these patients have dementia or MCI. This leads the physician to develop a heuristic about patients similar to Mary. Thus, when Mary exhibited some minor impairment in memory, the heuristic developed by her physician contributed to Mary being diagnosed with amnestic MCI. In contrast to the physician, the neuropsychologist working with Mary frequently encounters patients reporting clinically significant symptoms of depression and anxiety. More often than not, these patients experience problems with memory and/or attention. Consequently, the neuropsychologist developed a heuristic that borderline or mildly impaired memory may be a byproduct of depression and not necessarily caused by a neurodegenerative disorder. 
As can be seen, both the physician and neuropsychologist utilized prior experience in forming divergent viewpoints of Mary's condition.

\section{Clinical versus Statistical Judgment}

\section{The Legacy of Paul Meehl}

The question of clinical versus statistical judgment was first brought to prominence in 1954 after Paul Meehl published a book comparing the two methods. Meehl classified clinical judgment as judgments based on the prior experience of a human judge. On the other hand, human decision-making is eliminated in the statistical method in favor of empirical relations between obtained data and the criterion. But, basing decisions on a statistical model entails more than simply applying automated decision rules just as programming computers entails more than mimicking clinical judgment. The key point is that in order for a decision method to be considered statistical, it must not only be automated but must also be based on empirically established relationships (Dawes, Faust, \& Meehl, 1989).

Since Meehl's (1954) seminal publication, the number of investigations examining clinical versus statistical judgment has grown to more than 150 , and the results remain consistently in favor of the statistical method. In the few studies in which a preference for clinical judgment was noted, alterations to the statistics used in the prediction equation had a tendency to tip the scales in favor of the statistical method (Dawes, 2005). In sum, the role of clinical versus statistical judgment has been well studied in psychology, and the evidence points to the clear superiority of the statistical method (Dawes, Faust, \& Meehl, 1989). 


\section{Clinical Versus Statistical Judgment in the Diagnosis of MCI}

Returning to the diagnosis of Mary, it is apparent clinical judgment has led to two contradictory hypotheses made by her attending physician and neuropsychologist: (1) that she should be diagnosed with MCI because of her memory decrements and (2) that she should not be diagnosed with MCI because her suboptimal memory may be a byproduct of depression and anxiety. How such contradictory views can be formed has already been discussed, but a solution not formally proposed. As will be outlined in the next section of this paper, the lack of standardization of the existing diagnostic criteria needs to be addressed before the development of a statistical model designed to predict conversion from MCI to dementia.

Because of the ambiguity of diagnostic criteria, clinical judgment is heavily relied upon when determinations about an MCI diagnosis are made. This leads to a number of potential problems that affect both clinical practice and research studies: (1) people with MCI will not receive treatment until more serious cognitive decline is exhibited, (2) people will be misdiagnosed as having MCI and be given unnecessary treatment, (3) samples included in research studies will be representative of different populations leading to the decreased generalizability of the findings and how they relate to MCI, (4) the prognosis of MCI patients remains unclear, and (5) reliable estimates of MCI prevalence cannot be obtained. A statistical model can help in this regard. However, before a discussion of MCI diagnostic criteria and the application of a statistical model can take place, the concept of MCI as a predementia syndrome will be introduced. 


\section{Predementia: From "Normal" Cognition to Dementia}

As the brain ages, many older adults experience changes in cognition. It is only when changes exceed what is expected that normal cognition is thought to be compromised. As many as 1 in 7 adults over the age of 71 are currently living with dementia (Plassman et al., 2007), and this number may triple by the year 2050 with older adults over the age of 85 being most affected (Hebert, Scherr, Bienias, Bennett, \& Evans., 2003). Although numerous different dementia syndromes have been identified, Alzheimer's disease (AD) is the most well-known and widely researched type of dementia. But transition from normal cognition to AD is not a one-step process, and "preclinical stages" (changes in the brain that occur prior to the onset of clinically detectable symptoms) are thought to precede the disease and may last upwards of 20 years. Once these preclinical changes become associated with observable cognitive or functional deficits, a predementia syndrome is said to be present. To date, a number of predementia syndromes have been proposed, but relatively few have thoroughly delineated research criteria that yield the opportunity for ongoing study and the examination of rates of progression to dementia (Panza et al., 2005).

\section{Mild Cognitive Impairment: The Evolution of a Diagnosis Reflects Limitations}

Of all the terms used to describe predementia syndromes, the most well-known and extensively researched is MCI. Simply stated, MCI is a term used to describe cognitive decline that does not significantly interfere with daily functioning and, therefore, does not meet criteria for dementia. Given this broad definition, the potential exists for MCI to be a term that unifies all other terms used to classify predementia syndromes and declines in cognition not associated with normal aging. However, this 
was not always the case as memory impairment was originally a necessary diagnostic component. By having this stipulation, MCI was more reflective of a pre-AD stage than a preclinical syndrome related to general cognitive impairment. Currently, MCI is no longer conceptualized as a unitary construct dependent on memory impairment but a syndrome with multiple subtypes: amnestic-MCI (aMCI); multiple domains, slightlyimpaired-MCI (mdMCI); and single, non-memory-domain-MCI (snMCI).

\section{Conceptualized: Contributions of the New York University Group}

MCI was originally conceptualized by a research group from New York University in 1991 (Flicker, Ferris, \& Reisberg, 1991) using stages from the Global Deterioration Scale (Reisberg et al., 1982) (Panza et al., 2005). Six stages comprise the Global Deterioration Scale: Stage 1 (no cognitive decline), Stage 2 (very mild cognitive decline), Stage 3 (mild cognitive decline), Stage 4 (moderate cognitive decline), Stage 5 (moderately severe cognitive decline), and Stage 6 (severe cognitive decline). Although the Global Deterioration Scale was designed to assess degenerative dementia and describe its stages, it was noted by the group from New York University that a number of patients fell into stages 2 and 3 and that these patients were exhibiting mild cognitive impairment instead of dementia.

Diagnostic Criteria and Impairment Cutoffs: Contributions of the Max Planck I nstitute

As previously noted, diagnostic criteria were not proposed when the term MCI was conceptualized because the label of MCI was only applied to patients scoring in a designated range on a single measure (i.e., the Global Deterioration Scale). In 1992, Zaudig attempted to reclassify MCI according to existing diagnostic systems. After 
administering the Structured Interview for the Diagnosis of Dementia of the Alzheimer Type, multi-infarct dementia, and dementias of other etiology (SIDAM: Zaudig et al., 1991; Zaudig \& Hiller, 1991) to a sample of 150 randomly selected older adults living in Germany, patients with cognition anddaily functioning consistent with dementia and MCI were identified.

Using the Diagnostic and Statistical Manual of Mental Disorders, $3^{\text {rd }}$ Edition Revised (American Psychiatric Association, Diagnostic and Statistical Manual of Mental Disorders, $3^{\text {rd }}$ ed., revised [DSM-III R], 1987) criteria, two types of MCI patients were classified. Type 1 patients (MCI Type 1) exhibited characteristics identical to the DSMIII-R diagnostic criteria for Amnestic Syndrome: memory impairment (short- and longterm memory) but no impairments in any other cognitive domain. The other type (MCI Type 2) exhibited impairments to memory and another cognitive domain.

Using International Statistical Classification of Disorders (World Health Organization, International Statistical Classification of Disorders and Health Related Problems, $10^{\text {th }}$ Revision [ICD-10], 1992) criteria, three "types" of MCI patients were classified. Type 1 and Type 2 patients were identical to those outlined by DSM-III-R criteria. Type 3 patients (MCI Type 3), however, could display characteristics of either MCI Type 1 or MCI Type 2 along with accompanying deterioration in emotional control, social behavior, or motivation. Using these definitions in combination with performance on the SIDAM, optimal scores for characterizing patients as normal, MCI Type 1, MCI Type 2, MCI Type 3, and demented were reported. As can be seen, this work represented a critical step forward in advancing a statistical approach to diagnosing MCI. However, as will be detailed below, subsequent work did not capitalize on this. 


\section{Diagnostic/Research Criteria: The Contributions of Ronald Petersen}

In what has become known as the "Petersen criteria" or "Mayo criteria" (Petersen et al., 1997; Petersen et al., 1999) the first diagnostic/research criteria for MCI were developed: (1) memory complaint, (2) normal daily functioning, (3) normal general cognitive function, (4) abnormal memory for age, and (5) not demented (see Table 1). Although this represented an important advance in the development of the MCI construct, a number of limitations were present. First, memory impairment was deemed necessary, but many older adults with impaired cognition do not have a primary impairment in memory. Instead, many have primary impairment in other areas of cognition (e.g., executive dysfunction). Furthermore, evidence exists that in some patients, changes to executive function precedes the onset of changes in memory (Carlson et al., 2009). Second, clinical judgment is required on the part of the patient due to a subjective memory complaint being a necessary criterion. This means memory impairment need not only be present but also judged as present and reported by the patient. Third, "normal" daily functioning must be exhibited, but what constitutes "normal" remains a judgment of the clinician. Moreover, some declines in daily functioning are common in nondemented older adults, and this further complicates the reliance on clinical judgment. Finally, phrases such as "normal general cognitive function" and "abnormal memory for age" do not specify what constitutes normal from abnormal and, once again, lead to an overreliance on clinical judgment. Is 1.0 standard deviation (SD) below the mean abnormal? Is $1.5 \mathrm{SD}$ below the mean abnormal? Should age and education be taken into account? Answers to these questions are not clear from the criteria and, therefore, clinicians must use their best judgment to decide. 


\section{First Key Symposium}

At the First Key Symposium in Stockholm, Sweden, in 2003, it was

acknowledged that MCI could have multiple etiologies and subtypes that are not mutually exclusive and not necessarily related to a degenerative neurological disorder such as AD (Winblad et al., 2004) (see Table 2). Another recommendation from this consensus conference had to do with the second Petersen criteria (normal daily functioning). As a result, patients with early and subtle changes to daily functioning could still be considered for an MCI diagnosis. Moreover, a concrete definition of "subtle" was not explicitly stated and a description about how to go about assessing these subtle changes to daily functioning was not given. Consequently, the best way to assess for declines to daily functioning and the threshold for "subtle changes" remained highly influenced by clinical judgment.

\section{Latest Recommendations for Research/Diagnostic Criteria}

The most current recommendations for diagnostic/research criteria for MCI come from a workshop organized by the National Institute on Aging and Alzheimer's Association (NIA/AA) (Albert et al., 2011). These criteria are very similar to original Petersen criteria, and many of the issues regarding standardization and overreliance on clinical judgment found in the original Petersen criteria remain unchanged (see Table 3).

\section{Impairment in MCI: Overreliance on Clinical Judgment}

In the most recent recommendations given for MCI diagnostic criteria (Albert et al., 2011), impairment can be present in one or more cognitive domains (Table 3). The following description of impairment was given by the NIA/AA workgroup: "There should be evidence of lower performance that is greater than would be expected for the 
patient's age and educational background. If repeated assessments are available, then a decline in performance should be evident over time" (Albert et al., 2011, p. 271). A critique of this definition has to do with how much clinical judgment is needed to interpret the description. Specifically, what constitutes "lower performance?" Since it is not explicitly stated, this question is left to the judgment of the clinician. Moreover, reference is given to a "decline in performance over time" but no specifications as to what constitutes decline. Does this mean one, five, or ten percentage points in relation to age- and education-matched peers? Once again, this determination is left to the judgment of the clinician.

Later in the Albert et al (2011) paper, it is noted: "Cognitive testing is optimal for objectively assessing the degree of cognitive impairment for an individual. Scores on cognitive tests for individuals with MCI are typically 1 to $1.5 \mathrm{SD}$ below the mean for their age- and education-matched peers on culturally appropriate normative data (i.e., for the impaired domain(s), when available). It is emphasized that these ranges are guidelines and not cutoff scores" (Albert et al., 2011, p. 273). It is interesting that this recommendation is not included with the actual diagnostic criteria and that the impairment ranges are considered "guidelines and not cutoff scores." By setting clear impairment cutoffs, the diagnosis of MCI can be more standardized and, therefore, less reliant on clinical judgment. Moreover, the difference between a standard deviation of 1 $\mathrm{SD}$ and $1.5 \mathrm{SD}$ below the mean is a relatively wide range. In terms of percentiles, this places the range between the $7^{\text {th }}$ and $16^{\text {th }}$ percentile, meaning the patient would have to score worse than either $84 \%$ or $93 \%$ of the population after correcting for age and education. Consequently, even for clinicians choosing to use less clinical judgment by 
following the guidelines, judgments must be employed in order to choose which cutoffs to use.

Another problem with this description of impairment is the failure to take into account premorbid intelligence. Simply stated, premorbid intelligence is an estimate of a person's cognitive functioning before changes in cognition started to appear. This need to evaluate current cognition in relation to pre-morbid intelligence is highlighted by the Guidelines for the Evaluation of Dementia and Age-Related Cognitive Decline (American Psychological Association, 2011). Although these guidelines are described as aspirational and not enforceable, guideline 4 states that psychologists should attempt to estimate premorbid intelligence when measuring cognitive changes in individuals. Without an estimate of premorbid intelligence, determining a change in cognition is difficult and left to the judgment of the clinician. For instance, an older adult with an estimated pre-morbid Intelligence Quotient (IQ) falling into the $16^{\text {th }}$ percentile would be demonstrating no decline in relation to peers using a $1.0 \mathrm{SD}$ cutoff. However, the same older adult would be considered impaired using the recommendations from Albert et al. (2011). In contrast, interpretation would be quite different for an older adult with an estimated premorbid IQ falling at the $84^{\text {th }}$ percentile. For this patient, performance on cognitive measures falling at the $50^{\text {th }}$ percentile represents a fairly substantial decline in cognition, and performance falling into the $16^{\text {th }}$ percentile represents a change in cognition of two SD. Without taking into account pre-morbid intelligence, the same impairment threshold would be used when, in reality, the first patient is exhibiting no changes in cognition while the second patient is exhibiting drastic changes in cognition. 
Ideally, obtaining an estimate of premorbid IQ would be as easy as reading a WAIS report that was administered to the patient sometime in the past, but these kinds of records are rarely available. Instead, a neuropsychologist should evaluate a skill that is relatively resistant to the changes in cognition associated with $\mathrm{MCI}$ and dementia. This is most easily accomplished by evaluating reading ability because reading ability does not significantly differ in relation to peers even in older adults diagnosed with mild dementia (McGurn et al., 2004). However, minor changes in reading have been noted early in the course of dementia, and these changes increase as the disease progresses (Cockburn et al., 2000; O'Carroll et al., 1995; Patterson, Graham, \& Hodges, 1994). Consequently, for patients with moderate to severe dementia, reading ability cannot be relied upon to yield an accurate estimate of pre-morbid intelligence.

Measures such as the American National Adult Reading Test ANART (Nelson \& O’Connell, 1978) provide a good example of how to best assess reading to gain an estimate of pre-morbid intelligence. The ANART contains 50 low-frequency/irregular English words (e.g., ache and thyme), and the patient is instructed to read aloud each word with the number of words correctly pronounced comprising a total score. Normative data correcting for age, gender, and education are available (Kiely et al., 2011). Other techniques such as using a statistical model designed to estimate premorbid intelligence from demographic variables are available (Crawford, Millar, \& Milne, 2001). Although reading tests such as the ANART have been shown to yield the most accurate estimates of premorbid IQ, measures combining reading ability, best performance, and demographic variables are needed and have the potential to be more accurate than a test of reading ability used in isolation (Griffin et al., 2002). 
Turning again to the example case, it can clearly be demonstrated how clinical judgment must be used given the ambiguous nature of the current MCI diagnostic criteria. Even following the recommendations given by the Alzheimer's workgroup (1 $\mathrm{SD}$ to $1.5 \mathrm{SD}$ below the mean), clinical judgment is still needed in determining a diagnosis. Mary exhibited general cognitive functioning falling at the $25^{\text {th }}$ percentile and immediate memory falling at the $20^{\text {th }}$ percentile. No clinical judgment is needed because these scores are above both recommended cutoffs. However, she exhibited delayed memory falling at the $15^{\text {th }}$ percentile, and this score falls into the gray area between $1 \mathrm{SD}$ and 1.5 SD below the mean. Does this mean that impairment is present and a diagnosis of amnestic MCI applicable? Unfortunately, clinical judgment will be needed to decide. It is also difficult to ascertain the significance of Mary's scores because an estimate of premorbid intelligence was not obtained. Is Mary's premorbid intelligence average, below average, or above average? This information would certainly give a better perspective about her current scores, but this information is not available.

\section{NIA/AA Recommendations and Biomarkers and Imaging}

Criteria from the Alzheimer's Workshop are divided into two parts: one containing reference to biomarkers and imaging and one not containing reference to biomarkers and imaging. Biomarkers and imaging under examination and MCI criteria incorporating biomarkers and imaging are listed in Tables 4 and 5, respectively. Taken together, these criteria offer an exciting advancement in the diagnosis of MCI. However, the inclusion of biomarkers and imaging is still considered an exploratory step. As noted by Albert and colleagues, this inclusion is to "determine the likelihood of cognitive and functional progression for an individual MCI patient to a more severe stage of MCI or to 
dementia, and the likelihood that this progression will occur within a defined period" (pg. 275). Albert and colleagues go on to note that imaging and biomarkers are not validated in MCI and that studies are largely confined to the examination of patients with AD. For studies examining MCI patients, few have compared biomarkers and imaging with each other or with histopathologic confirmation. Furthermore, studies combining multiple biomarkers and imaging data have not been conducted. Taken together, this means that predictive studies regarding imaging and biomarkers in relation to MCI are greatly needed to advance diagnostic accuracy. (A brief review of imaging and biomarkers in $\mathrm{AD}$ can be found later in this proposal).

\section{MCI: Estimates of Prevalence and Incidence are Inconsistent}

Earlier it was stated that one effect of clinical judgment could be that the samples used in MCI research may, in fact, be representative of quite different populations. This critique is well supported by research on the prevalence and incidence of MCI in the general population. From 1995-2009, nine studies on MCI incidence rates were conducted in North America, South America, and Europe. Although each of these nine studies used similar diagnostic criteria, application of these criteria varied from study to study. As noted in a review by Luck et al. (2010), MCI diagnostic criteria were modified in four of the nine studies. In three studies, a subjective report of cognitive impairment was not required, and in one study a subjective report of cognitive impairment and preserved daily functioning were not required (the daily functioning criteria used in this study is similar to the most recent diagnostic recommendations made by the Alzheimer's workgroup.). Impairment thresholds also varied among studies. Performance less than 1.5 SD below the mean, less than 1.0 SD below the mean, and less than the $10^{\text {th }}$ 
percentile in relation to age-, education-, and sometimes gender- and race-corrected scores were all used (Luck et al., 2010).

Given the wide array of differing applications of the MCI diagnostic criteria in incidence studies (both for Petersen and MCI-R criteria), it is not surprising the range of estimates of MCI incidence is quite large. For any MCI type, estimates range from 8.576.8 per 1,000 person-year (Ravaglia et al., 2008; Solfrizzi et al, 2004; Tervo et al., 2004; Chaves et al., 2009; Caracciolo et al., 2008; Manly et al., 2008; Verghese et al., 2006; Busse et al., 2003; Larrieu et al., 2009; Lopez et al., 2003). Estimates for amnestic MCI range from 8.5-40.6 per 1,000 person-year while estimates of non-amnestic MCI range from 28-36.3 per 1,000 person-year. Higher estimates were noted in studies that did not require a subjective report of cognitive impairment. As can be seen from these estimates, the incidence of MCI in the population is not well understood and reflects a need for the standardization of the diagnostic criteria. Age, education, and vascular risk factors were consistently associated with increased incidence of MCI. No studies on the incidence of MCI using criteria proposed by the Alzheimer's workgroup have yet been conducted.

Several studies have been conducted comparing the prevalence of MCI in the population according to both MCI and MCI-R criteria. As with the incidence studies discussed above, the interpretation of the criteria vary from study to study and also highlight the need for standardized criteria. For instance, in a sample of 3,327 older adults over the age of 75 who were receiving care from a general practitioner, the prevalence of MCI was $15.4 \%$ using Petersen criteria and $25.2 \%$ using MCI-R criteria (Luck et al., 2007). The SIDAM was used to evaluate cognitive impairment, and domain specific cognitive impairment was considered present if performance fell more than $1 \mathrm{SD}$ 
below the mean using age- and education-corrected scores. The SIDAM-ADL scale was used to evaluate daily functionin, and patients with one or no impairments on the 14 SIDAM-ADL items were considered functionally unimpaired. Significant risk factors included age, vascular disease, depression, and APOE4 genotype.

In a study of 883 older adults over the age of 60 receiving care from a general practitioner, the prevalence of MCI was $16.6 \%$ using MCI-R criteria but fell to $3 \%$ when using Petersen criteria (Artero et al., 2006). A computerized neuropsychological evaluation assessing a number of cognitive domains was used to evaluate for cognitive impairment. Patients were considered impaired if performance fell more than $1.5 \mathrm{SD}$ below age- and education-corrected scores on a memory or other cognitive task. Daily functioning was required to be "essentially normal" (Artero et al., 2006, p. 467). However, specifics about the evaluation of daily functioning were limited to the following quote: "A validated activity of daily living scale with finely graded hierarchical subscales was used to assess alterations in everyday functioning in collaboration with both subjects and caregivers" (Artero et al., 2006, p. 466).

The two studies outlined above were chosen to highlight how lack of standardized criteria can lead to differing estimates using Petersen and MCI-R criteria in a similar sample (i.e., patients of general practitioners). In the study by Luck et al (2007), the authors judged impairment to be present if scores fell more than $1 \mathrm{SD}$ below the mean using age- and education-corrected scores on a neuropsychological test. However, in the Artero and colleagues' study, the authors did not judge impairment to be present until age- and education-corrected scores were more than $1.5 \mathrm{SD}$ below the mean. Evaluation of daily functioning was also not uniform between the two studies. In Luck and 
colleagues' study, a patient's daily functioning was judged as impaired if difficulties in two or more areas were displayed. However, daily functioning was required to be essentially normal by Artero and colleagues. Although comparisons between the prevalence estimates noted in these two studies are not appropriate due to the difference in ages of the samples (75+ vs. $60+)$, it can easily be seen how clinical judgment would lead to different prevalence estimates even when using an identical sample. No studies on the prevalence of MCI using criteria proposed by the Alzheimer's workgroup have yet been conducted.

\section{Conversion from MCI to Dementia: Varying Estimates}

As with estimates of incidence and prevalence, the role of clinical judgment in the interpretation of $\mathrm{MCI}$ diagnostic criteria has contributed to uncertainty regarding prognosis. For some patients diagnosed using current criteria, an improvement in cognition can be expected. For others, cognition will remain stable. For still others, cognition will continue to decline until a diagnosis of dementia is warranted. However, these estimates vary widely in the research literature. A review of studies of dementia conversion well illustrates this point.

In a study using original Petersen criteria, $32.2 \%$ of patients diagnosed with MCI progressed to dementia within two years (Amieva et al., 2004). In this study, general cognitive functioning was judged to be intact for patients with at least a primary school education and scoring $>25$ on the MMSE. For patients with less education, general cognitive functioning was judged as intact if scores were $>23$ on the MMSE. For both education groups, domain specific impairment was judged as present if scores were more than 1.5 SD below the mean using age- and education-corrected scores on either a test of 
working memory or semantic fluency. No dependency for basic ADLs and slight dependency for higher-order ADLs were required for an MCI diagnosis. In another study in which a differing interpretation of original Petersen criteria was used (i.e., MMSE scores $<25$ regardless of education for intact general cognitive functioning, 1.0 SD cutoff for domain specific impairment, and normal daily functioning), a conversion rate of $27 \%$ was noted over the course of ten years, and only $15 \%$ of patients progressed to dementia over the first two years (Ganguli et al., 2004).

MCI-R criteria have been noted as exhibiting better predictive validity of conversion to dementia (sensitivity of $95 \%$ and specificity of $66 \%$ ) than the original Peterson criteria (sensitivity of 5\% and specificity of $91 \%$ ) when the same impairment cutoffs are used for both groups (Artero et al., 2006). However, issues are also present with MCI-R criteria as the application of these criteria can lead to strikingly different conversion estimates. For instance, when using cutoffs of more than 1 SD and more than 1.5 SD below the mean on the SIDAM for both Petersen and MCI-R criteria, the best predictive validity (sensitivity of $74 \%$ and specificity of $73 \%$ ) was observed when MCI-R criteria was coupled with a 1 SD cutoff (Busse et al., 2006). When using a 1.5 SD cutoff, specificity increased to $86.1 \%$ but at the expense of a decrease in sensitivity to $40.4 \%$. Overall, conversion rates varied by $45 \%$ depending on which diagnostic criteria were used and how they were applied. No studies of rates of conversion from MCI to dementia using criteria proposed by the Alzheimer's workgroup have yet been conducted. 


\section{Proposing an Statistical Model: A Two-Step Process}

\section{The Two Steps}

As outlined in Tables 2 and 3, the etiology of MCI (i.e., degenerative, vascular, metabolic, traumatic, psychiatric, and other) can vary from patient to patient. These etiologies also have significant long-term implications. For example, if the cause of MCI is a degenerative brain disorder (i.e., $\mathrm{AD}$ or other types of dementia), then $\mathrm{MCI}$ is considered a predementia syndrome and will continue to progress until the death of the patient; however, if the cause is reversible (i.e., metabolic and psychiatric), treatments can be applied to help the patient's cognition remain stable or improve. As a concept, MCI is designed to identify patients in the first category: those who will eventually go on to develop a dementia syndrome. But in practice, patients in both groups are diagnosed with MCI. This means patients with cognitive decline stemming from depression, anemia, thyroid disease, and/or a vitamin deficiency will be diagnosed the same as patients in the preclinical stages of a dementia syndrome.

Using a statistical model, MCI patients could be classified based upon risk for conversion. Essentially, this would divide MCI into two groups based upon risk determined by comparison to a single cut-score. For one group, risk for conversion would be high. For the other group, risk for conversion would be low. Furthermore, through the application of screening and conversion models (explained below), issues present in current research studies could be mitigated to a large degree. To construct such a model, two steps are needed. Step 1 would involve constructing a "screening" model, and Step 2 would involve constructing a model to predict conversion. 


\section{Step I: Screening Patients for Application of a Conversion Model}

A screening model used to identify patients to be evaluated by a conversion model should be weighted in favor of including false-positives over false-negatives. This is because a screening model is aimed at determining which individuals need a more thorough and expensive evaluation so the conversion model can be applied. However, for the purpose of ease and consistency among samples used in research studies, the importance of using standardized criteria with the least amount of reliance on clinical judgment cannot be overstated (see Table 6 for recommendations).

First, it is recommended that patient judgment (subjective complaints), family judgment (family reports of decline), informant judgment (reports from non-family members that decline is present), and clinical judgment (reports from providers) be excluded from a screening model. Second, objective evidence of impairment should be standardized and pre-morbid IQ taken into account. As recommended by Busse et al. (2006), a cutoff of 1.0 SD below the mean should be used to best optimize the number of older adults identified as at risk for conversion to a dementia syndrome. Although this cutoff inflates the number of false-positives to a degree, it reduces the risk of not identifying patients who will later go on to develop dementia. Given the opportunity to treat and study these predementia patients, the increased rate of false-positives seems an acceptable compromise. Moreover, application of the second model (i.e., conversion model) would further mitigate the increased rate of false-positive results. In other words, those who were falsely identified as MCI by the simple screening model could later be correctly identified using the more complex conversion model. Third, an older adult should not be considered impaired if scores do not fall more than 1.0 SD below estimates 
of pre-morbid IQ. As outlined earlier in this proposal, older adults who exhibited cognitive abilities at the $10^{\text {th }}$ percentile when they were 20 years old should not be considered impaired if performance falls at the $10^{\text {th }}$ percentile when they are 70 years old. Fourth, functional abilities and daily functioning should be evaluated with a functional measure validated on the patient population being assessed. Moreover, essentially normal daily functioning should not be required because older adults can experience some changes in functional abilities not related to cognitive impairment and, therefore, not indicative of dementia (similar to MCI-R criteria). With these recommendations, clinical judgment can be taken out of a screening model. Furthermore, the simplicity of the proposed screening model would work to lessen the constraints of human cognitive limitations.

The screening model based on the above recommendations would have four components: (1) scores $>1$ SD below mean age- and education-corrected scores on a test of general cognitive functioning (i.e., ADAS-COG); (2) scores $\leq 1$ SD below mean ageand education-corrected scores on a measure evaluating a specific cognitive domain (i.e., memory); (3) performance on domain specific test is $\leq 1.5 \mathrm{SD}$ below estimate of premorbid intelligence; and (4) no difficulty or mild difficulty in any daily functioning domain as determined by scores on an empirically validated functional measure.

By using Mary's scores corrected for age and education as an example, it can be demonstrated how this screening model could be used for amnestic MCI. On a test of general cognitive functioning, Mary scored in the $25^{\text {th }}$ percentile. This score indicates that her general cognitive functioning is not considered impaired so $a+$ is noted for the first part of the model. On tests of memory, she scored in the $20^{\text {th }}$ percentile for Total 
Recall and in the $15^{\text {th }}$ percentile for Delayed Recall. Her score on Delayed Recall is $\geq 1$ SD below the mean so $\mathrm{a}+$ is also noted for the second part of the model (domain specific impairment). Although not administered an empirically validated functional measure, Mary's daily functioning was reported as normal so a + is noted for the fourth part of the model. However, by relying on family reports, family judgment has been used to determine if a + was warranted for the parts of the equation related to ADL and IADL functioning. This emphasizes the importance of using an empirically validated functional measure as part of a neuropsychological assessment so clinical judgment can be taken out of the equation.

So far, Mary has received a + on all addressed parts of the model. However, Mary's premorbid intelligence was not assessed. Because of this oversight, the screening model cannot be completed. If, for example, Mary's performance on the ADAS-COG $\left(25^{\text {th }}\right.$ percentile) is reflective of her premorbid intelligence, she would receive a - for the third part of the equation because her Logical Memory score is within 1 SD. In contrast, $\mathrm{a}+$ would be warranted if her performance on the ADAS-COG is not reflective of her premorbid intelligence. For example, if Mary's premorbid intelligence fell at the $67^{\text {th }}$ percentile, her score on Delayed Recall would be more than 1 SD lower. Consequently, a + would be noted for the third part of the model. However, the screening model is only a step to identify people most in need for evaluation from the second (conversion) model. If any question arises about a patient, the steps needed to apply the second model should be taken. For example, the screening model was not able to be fully completed for Mary because her premorbid intelligence was not evaluated. However, a discrepancy was noted between scores on tests of general cognitive functioning and memory. 
Consequently, it is recommended that Mary take the steps needed for the conversion model to be applied.

\section{Step II: Constructing a Model to Predict Conversion to Dementia}

Statistical models for predicting dementia risk in the older adult population can be found in the literature. The models with the best predictive validity are multifactorial and use data from neuropsychological testing, patient and informant report, imaging, genetics, and other demographic and health information. The most complex of these models is the Late-Life Dementia Risk Index (Barnes et al., 2009). This index uses age, cognition (scores on two short screens), body mass index, ApoE4, imaging, vascular risk factors, functional abilities, and factors related to lifestyle to generate placement in three groups: low-risk (index score of 0-3), moderate-risk (index score of 4-7), and high-risk (index score $\geq 8$ ). Although models such as the Late-Life Dementia Risk Index exist, their utility is limited because in clinical practice it is extremely rare to have access to information about so many risk factors, and many steps would be needed to gain information for the average patient. This demonstrates two needs: (1) models need to be designed that target MCI patients instead of the general population, and (2) standardized criteria need to be developed that identify at risk patients so a conversion model can be applied. However, to date no multifactorial conversion models of this type have been developed.

As can be seen from the Late-Life Dementia Risk Index, biomarker and imaging data are important parts of a comprehensive model designed to predict dementia risk. However, as noted earlier, research regarding biomarkers and imaging in relation to $\mathrm{MCI}$ is in its infancy. For the current study the following biomarkers and types of imaging are 
candidates for inclusion in a statistical model designed to predict conversion from MCI to AD: (1) Homocysteine is a non-protein amino acid and associated with AD in studies comparing autopsied patients with AD versus controls (reviewed in Frank et al., 2003). Increased homocysteine levels are associated with a four times greater risk for AD; (2) Oxidative/nitrative damage is associated with $\mathrm{AD}$, and specific isoprostanes are elevated in the urine, blood, and CSF of AD patients. Moreover, oxidative/nitrative damage is correlated with memory impairments, CSF tau levels, and the number of APOE4 alleles (Frank et al., 2003; Pratico et al., 2000; Pratico, Clark, Liun, Lee, \& Trojanowski, 2002; Pratico, Uryu, Leight, Trojanoswki, \& Lee, 2001; Pratico, V, Trojanowski, Rokach, \& Fitzgerald, 1998; Reckess, 2003; Yao et al., 2003); (3) Recent studies have shown that sulfatide (a major constituent of brain lipids) may be associated with AD (reviewed in Reckess, 2003). Sulfatide decreases up to $93 \%$ in gray matter and $58 \%$ in white matter in MCI versus controls. Furthermore, CSF sulfatide may differentiate non-demented individuals from those with very mild dementia; (4) Tau and $A \beta$ (components of tangles and plaques, respectively) are the most commonly studied potential AD biomarkers. CSF tau levels are associated with AD pathology (decreased in patients with AD), and CSF tau levels aid in the differential diagnosis of $\mathrm{AD}$ from other dementing disorders (Arai et al., 1998; Arai, Morikawa et al., 1997; Arai et al., 1995; Araiet al., 1997; Clark et al., 2003; Frank et al., 2003; Reckess, 2003); (5) Using MRI images, it has been shown that neurodegeneration of the medial temporal region and specifically the left hippocampus can be used to predict conversion from MCI to dementia (Risacher et al., 2009); (6) Abnormal patterns of glucose use have been associated with AD. Using PET images, it has been shown that reduced glucose in the limbic structures and in particular the 
hippocampus is associated with MCI and subsequent conversion to dementia (reviewed in Schuff \& Zhu, 2007). 


\section{RESEARCH QUESTIONS AND HYPOTHESES}

The current study will address the following research questions:

1. Can demographic factors be used to predict conversion from MCI to dementia? It is hypothesized the female gender and low education will yield the highest predictive values.

2. Can neuropsychological testing be used to predict conversion from MCI to dementia? It is hypothesized Logical Memory - II will yield the highest predictive value of any measure.

3. Can biomarkers collected from CSF, urine, or blood be used to predict conversion from MCI to dementia? It is hypothesized homocysteine levels will yield the highest predictive value.

4. Can brain imaging be used to predict conversion from MCI to dementia? It is hypothesized hypometabolism in the hippocampal brain area will yield the highest predictive value.

5. Can daily functioning be used to predict conversion from MCI to dementia? It is hypothesized performance on tests of neuropsychological functioning will be a better predictor of conversion than daily functioning. 6. Can neuropsychiatric functioning be used to predict conversion from MCI to dementia? It is hypothesized depression will yield the highest predictive value of any behavioral abnormalities. 
7. Can data from neuropsychological testing, demographic variables, biomarkers, brain imaging, daily functioning, and neuropsychiatric functioning be used to construct a statistical model that predicts conversion from MCI to dementia with a high level of accuracy? Furthermore, can this model be used to predict conversion from MCI to dementia better than predictions made from any single variable or variables from any factor groups (i.e., neuropsychological testing, demographic variables, biomarkers, and brain imaging)?

8. What diagnostic criteria can be used to construct an optimal screening model? For example, does a 1.0 SD or 1.5 SD cutoff using age- and educationadjusted scores on tests of neuropsychological functioning yield more acceptable inclusion rates in application of the screening model? Also, does using premorbid intelligence increase the psychometric properties of a screening model? Finally, does allowing for some minor difficulties with ADLs yield a more optimal screening model? It is hypothesized that using a $1.0 \mathrm{SD}$ cutoff will be preferable to using a $1.5 \mathrm{SD}$ cutoff, that taking premorbid intelligence into account will increase the psychometric properties of the screening model, and that allowing for some minor trouble with ADLs will further increase the psychometric properties of a screening model. 


\section{METHODS AND MATERIALS}

\section{Subjects}

Data for the current study came from the publicly available portions of the Alzheimer's disease Neuroimaging Initiative (ADNI). The ADNI is a non-randomized, natural history non-treatment study of biological and neurocognitive factors in MCI. In total, 800 subjects (200 normal controls, $400 \mathrm{MCI}$, and 200 mild AD) were recruited from 50 sites in the United States and Canada. Subjects were between the ages of 55-90 at the time of inclusion and required to provide an informant who could answer questions about the subject's general functioning. All subjects were required to consent to all test procedures and agree to longitudinal follow-up. No subjects were included who were currently taking psychoactive medications. The conversion from normal to MCI or AD and conversion from $\mathrm{MCI}$ to $\mathrm{AD}$ is of considerable interest. ADNI researchers estimate the most frequent conversion will be from MCI to AD and will occur at approximately $10-15 \%$ per annum.

For ADNI criteria, normal controls were required to score between 24-30 on the MMSE, have a Clinical Dementia Rating (CDR) score of 0, and be non-depressed. MCI subjects were required to score between 24-30 on the MMSE, have a history of a memory complaint (from the subject or informant), fall below education-adjusted cutoff scores on the Logical Memory II subscale from the Wechsler Memory Scale - Revised ( $\leq 8$ for 16 or more years of education, $\leq 4$ for $8-15$ years of education, and $\leq 2$ for $0-7$ years of 
education), have a CDR of 0.5 , have no impairment in domains other than memory and essentially normal ADLs. Mild AD subjects were required to have a history of a memory complaint (from subject or study partner) and abnormal memory function as indicated by education adjusted cutoffs on the Logical Memory II subscale from the Wechsler Memory Scale - Revised (same cutoffs used for MCI), MMSE between 20 and 26, CDR of 0.5 or 1.0. They also had to meet National Institute of Neurological and Communicative Diseases and Stroke/Alzheimer's Disease and Related Disorders Association (NINCDS/ADRDA) criteria for probable AD (McKhann et al., 1984). These criteria are as follows: memory impairment established by neuropsychological testing (using the standardized test battery), deficits $\left(\leq 5^{\text {th }}\right.$ percentile using age- and educationcorrected scores) in two or more areas of cognition, evidence of continued decline from a previous level of functioning through a collateral source and structured clinical examination or assessment of activities of daily living, no disturbance of consciousness, onset between ages 40 and 90, and the absence of systemic disorders or other brain diseases that could account for the progressive deficits in memory and cognition. These criteria have been shown to achieve good reliability and validity in the diagnosis of AD when compared with autopsy confirmed cases (Blacker et al., 1994).

To retain a high level of consistency between diagnostic categories, attempts were made to use ADNI criteria whenever possible. Consequently, the same cutoffs on the MMSE and CDR were used across diagnostic category. Four distinct samples were utilized for this study: patients identified as MCI using ADNI criteria, patients identified as MCI according to a $1.0 \mathrm{SD}$ cutoff on Logical Memory Delayed and $<6$ on the FAQ (proposed criteria \#1), patients identified as MCI according to a 1.5 SD cutoff on Logical 
Memory Delayed and $<6$ on the FAQ (proposed criteria \#2), and patients identified as MCI using a 1.5 SD cutoff in relation to VIQ and $<6$ on the FAQ (proposed criteria \#3). Criteria used for each diagnostic classification system can be found in Table 7.

\section{Procedures}

After baseline visits were complete, an experienced neurologist or psychiatrist determined the best diagnosis (normal, MCI, AD, or other). The diagnosing physician reviewed the medical history and all information gathered at the baseline visits to determine if there was significant impairment or deterioration. Based on the results of the clinical, neuropsychological, and laboratory information, the physician may have made a diagnosis and, if appropriate, further classified the diagnosis of AD into Probable AD or Possible AD. For the purposes of the ADNI database, Possible AD refers to situations where a second disorder is present that may cause dementia but is not considered to be the primary cause of the dementia (i.e. AD is considered to be the primary cause). If the subject had developed another diagnosis other than AD which is believed to be the primary etiology for cognitive impairment or dementia, this was also be specified.

At baseline, subjects underwent clinical and cognitive assessments. Structural magnetic resonance imaging (1.5T MRI) was conducted at designated intervals for the length of study inclusion (2-3 years) for all subjects. Approximately $50 \%$ of subjects also had Fluoro Deoxy Glucose Positron-Emission Tomography (FDG PET) scans at the same designated intervals. An additional $25 \%$ of subjects were not scanned using PET had MRI at 3 Tesla for greater image resolution. All subjects were evaluated at $0,6,12$, and 24 month intervals. In addition, MCI subjects were evaluated at 18 and 36 months, and 
normal controls were evaluated at 36 months. IRB approval was obtained at all affiliated sites, and signed consent was required from all subjects and informants before inclusion. Further details about study procedures including pre-screening, data management, and quality control can be found online at http://www.adniinfo.org/Scientists/Pdfs/adni_protocol_9_19_08.pdf.

\section{Demographic Variables of Interest}

A number of studies have found education to protect people from developing dementia (Brayne et al., 2010; Del Ser et al., 1999; Letenneur et al., 1999; Ott et al., 1995; Stern et al., 1994; Valenzuela et al., 2008; Valenzuela and Sachdev, 2006). Results of some studies indicate that females are more likely to develop dementia than males with gender differences becoming more pronounced for people over the age of 85 (Anderson

et al., 1999; Brayne et al., 1995; Clark et al., 1996; Fratiglioni et al., 1997; Morgan et al., 1993; Yoshitake et al., 1995).

\section{Measure of Dementia Severity}

\section{Clinical Dementia Rating (CDR):}

The CDR (Berg, 1988) describes five degrees of impairment in performance on each of 6 categories: memory, orientation, judgment and problem solving, community affairs, home and hobbies, and personal care. The ratings of degree of impairment obtained on each of the 6 categories of function are combined into one global rating of dementia (ranging from 0 to 3 ). The CDR has been shown to be reliable and valid for use with both diagnosis and staging in AD patients (Morris, 1997). 


\section{Measures of Neuropsychological Functioning}

\section{Mini-Mental State Examination (MMSE):}

The MMSE (Folstein et al., 1975) is a brief screening measure of general cognitive functioning and assesses the following cognitive domains: orientation, registration, short-term recall, attention, calculation, visuo/constructional skills and praxis. Total scores range from $0-30$. Various cut-scores have been used to classify levels of cognitive impairment with scores $\leq 24$ typically being indicative of cognitive impairment (Tombaugh \& McIntyre, 1992). Normative data correcting for age and education is also available (Crum et al., 1993). Although demonstrating utility as a short screening measure for cognition (Clark et al., 1999), the MMSE is not an accurate standalone measure for the diagnosis of AD (Tierney et al., 2000)

\section{$\underline{\text { Alzheimer's Disease Assessment Scale-Cognitive (ADAS-COG): }}$}

The ADAS-COG (Rosen, Mohs, \& Davis, 1984) is a structured measure that evaluates the following domains: memory (word recall and word recognition), reasoning (following commands), language (naming and comprehension), orientation, ideational praxis (placing a letter into an envelope) and constructional praxis (copying geometric designs). Ratings of spoken language, language comprehension, word finding difficulty, and ability to remember test instructions are also obtained. The number of errors made determines overall test score (0-70). Higher scores indicate greater impairment.

\section{Logical Memory Test (Delayed Paragraph Recall):}

The Logical Memory test that was administered is a modification of the episodic memory measure from the Wechsler Memory Scale-Revised (WMS-R) (D Wechsler, 1987). Free recall of one short story (Story A) consisting of 25 bits of information was 
recalled by the subject immediately after verbal presentation. A delay condition of 30 minutes is also included. The total bits of information from the story that are recalled immediately $($ maximum score $=25)$ and after the delay interval $($ maximum score $=25)$ are recorded. A retention or "savings" score can be computed by dividing the score achieved during delayed recall by the score achieved during immediate recall.

\section{Boston Naming Test (BNT):}

The BNT (Kaplan, Goodglass, \& Weintraub, 1983) is a measure of visual confrontational naming. Subjects are required to name objects depicted in line drawings. For the ADNI, only 30 items were presented (either the odd- or even numbered items from the full 60 -item test). The drawings are graded in difficulty with the easiest drawings presented first. If a subject encounters difficulty in naming an object, a stimulus/semantic cue (e.g., it is found in Egypt) and/or a phonemic cue (saying the first phonemes of the word) is provided. The number of spontaneous correct responses (maximum score $=30$ ) and spontaneous plus semantically-cued correct responses (maximum score $=30)$ are recorded.

\section{Category Fluency:}

Category Fluency (Butters, Granholm, Salmon, Grant, \& Wolfe, 1987) is a test of verbal fluency. Subjects are instructed to generate as many examples as possible from each of two semantic categories (animals and vegetables) in a 60-second time period. Performance is measured by the number of correct, unique examples generated for the two categories. Perseveration (repetitions of a correct item) and intrusion (non-category items) errors are also noted. 


\section{Clock Drawing Test:}

The Clock Drawing Test (Goodglass \& Kaplan, 1983) is a test of visuoperceptual/construction skills and has two conditions: command and copy. In the command condition, the subject is given a blank sheet of $81 / 2$ " X 11 " paper and instructed to "Draw a clock, put in all of the numbers, and set the hands for 10 after 11." After that task is completed, the subject is asked to copy a clock drawing. A quantitative score (maximum total score $=10$ ) is derived for each drawing by adding the scores of three separate features: 2 points for the integrity of the clock face, 4 points for the presence and sequencing of the numbers, and 4 points for the presence and placement of the hands. The Clock Drawing Test is effective for discriminating between subjects with AD and normal elderly individuals (Cahn et al., 1996).

\section{Digit Span:}

The Digit Span subtest from the WAIS-R (Wechsler, 1981) requires the subject to recall a series of single digits that were orally presented. In the Forward condition, the subject must repeat the digits in the same order. In the Backward condition, the digits must be repeated in the reverse order. Testing is terminated when the subject misses both trials at a given sequence length. A point is awarded for each correct sequence. Maximum score range is between 0-14.

$\underline{\text { Rey Auditory Verbal Learning Test (AVLT): }}$

The AVLT (Rey, 1964) is a test of learning and memory. Fifteen unrelated nouns are verbally presented (one per second) across 5 learning trials. The number of correctly recalled 
words on each trial is recorded. Following a 20-minute delay, the subject is asked to recall as many of the 15 words as possible. Directly following the delayed recall condition, a yes/no recognition test is administered. The number of target "hits" and false positive responses are recorded. Two equivalent alternate forms of the test were used across test sessions so that subjects were exposed to the same word list as infrequently as possible.

\section{Trail Making Test:}

The Trail Making Test (Reitan, 1958) consists of two parts: Part A and Part B. For Part A the subject is instructed to connect 24 circles with a drawn line as quickly as possible in ascending numerical order. For Part B, the subject is asked to connect the circles while alternating between numbers and letters in an ascending order (e.g., A to 1; 1 to $\mathrm{B}$; $\mathrm{B}$ to $2 ; 2$ to $\mathrm{C}$ ). The subject's performance is judged in terms of time (in seconds) required to complete each trail and by the number of errors of commission and omission. The time to complete Part A (150 second maximum) and B (300 second maximum) will be the primary measures of interest. Although both Parts A and B depend on visuomotor and perceptual-scanning skills, Part B also requires considerable cognitive flexibility in shifting from number to letter sets under time pressure. Both parts of the Trail-Making Test are available in multiple forms of equal difficulty for purposes of repeated evaluation.

\section{Digit Symbol Substitution:}

Digit Symbol Substitution (Wechsler, 1981) is a subtest from the WAIS-R that consists of 110 small blank squares (presented in seven rows), each randomly paired with one of nine numbers ( 1 to 9 ) printed directly above it. Above the row of blank squares is 
a printed "key" that pairs each of the numbers 1 through 9 with an unfamiliar symbol. Following a short series of practice trials, the subject must use the key to fill in the blank squares in order (working left to right across the rows) with the symbol that is paired with the number above it working as quickly as possible for 90 seconds. The number of blank squares filled in correctly within the time limit is the measure of interest (maximum raw score $=110$ ). This test engages multiple cognitive abilities including attention, psychomotor speed, complex scanning, visual tracking, and immediate memory.

\section{Measure of Premorbid Intelligence}

\section{American National Adult Reading Test (ANART):}

The ANART (Nelson \& O’Connell, 1978) is a test of reading used as an estimate of premorbid verbal intelligence. For the ANART, patients are required to read and correctly pronounce 50 "irregular" words that do not follow common rules of

phonography and orthography. Consequently, correct pronunciation depends on previous familiarity with the word. The 50 irregular words of the ANART are printed on a single sheet of paper which is presented to the subject who is instructed to read each word aloud. The number of mispronounced words is recorded by the examiner (maximum errors $=50$ ). Premorbid verbal intelligence can be estimated by applying a formula derived by Grober and Sliwinski: (118.2 - .89 (AMNART errors) +.64 [years of education]) (Grober \& Sliwinski, 1991).

\section{Biomarkers}

Homocysteine is a non-protein amino acid implicated in AD.

Oxidative/nitrative damage is associated with $\mathrm{AD}$ and can be evaluated using the blood, urine, and CSF of patients. 
Sulfatides are important components in muscle and nerve cell membranes and have been implicated in $\mathrm{AD}$.

Tau and $\mathrm{A} \beta$ are components of tangles and plaques, respectively and have been highly implicated in AD.

Neuroimaging

MRI scans use a large magnet, radio waves, antenna, and computer to produce clear images of the brain. By using MRI, invasive methods such as X-rays can be bypassed. Using MRI images, it has been shown that neurodegeneration of the medial temporal region and specifically the left hippocampus can be used to predict conversion from MCI to dementia (Risacher et al., 2009).

PET images can be used to detect how the brain uses glucose. Abnormal patterns of glucose use have been associated with AD.

\section{Measure of Functional Impairment}

Functional Activities Questionnaire (FAQ):

For the FAQ (Pfeffer, Kurosaki, Harrah, Chance, \& Filos, 1982), a caregiver or qualified partner is asked to rate the subject's ability to carry out ten complex ADLs: (1) manage finances, (2) complete forms, (3) shop, (4) perform games of skill or hobbies, (5) prepare hot beverages, (6) prepare a balanced meal, (7) follow current events, (8) attend to television programs, books or magazines, (9) remember appointments, and ((10) travel out of the neighborhood. Each activity is rated as 0 (does without difficulty), 1 (needs frequent advice or assistance), or 2 (someone has taken over the activity). Scores are summed across items to provide a total disability score (higher scores $=$ greater impairment; maximum score $=20$ ). If an activity was never or very rarely performed 
premorbidly, it is not rated and a pro-rated proportional score can be derived (achieved score / [20 - 2 times the number of items rated as never performed]). Using the FAQ, scores $>5$ have been shown to have predictive validity when distinguishing MCI from mild AD (Teng et al., 2010).

\section{Measures of Emotional and Behavioral Functioning}

\section{Neuropsychiatric Inventory Q (NPIQ):}

The Neuropsychiatric Inventory (NPI) is used to assess both the frequency and severity of behavioral abnormalities across 10 domains: delusions, hallucinations, agitation, depression, anxiety, euphoria, apathy, disinhibition, irritability, and aberrant motor behavior. Caregivers are asked to rate affected behaviors on a 1-4 scale for frequency (1 occasionally, 2 often, 3 frequently, and 4 very frequently) and a 1-3 scale for severity ( 1 mild, 2 moderate, 3 severe). Each domain is calculated by multiplying frequency and severity. NPI Total score (range $=0-12)$ is the product of the 10 domains designed to evaluate for behavioral abnormalities. The NPIQ (Kaufer et al., 2000) is a shorter version that does only the screening questions and the severity rating for each domain. The maximum score is 36 .

\section{Geriatric Depression Scale:}

The Geriatric Depression Scale (Short Form) (Sheikh \& Yesavage, 1986) is a self-report measure of depression specifically designed for use with an older adult population. The scale consists of 15 printed questions that the subject is asked to answer by circling yes or no on the basis of how he/she felt over the past week. One point is given for each positive or negative answer that is indicative of a symptom of depression, 
for a possible total of 15 points. Total scores of 0-5 are considered normal, and scores of 6-15 are considered evidence of clinically significant depression.

\section{Statistical Analyses}

Statistical analyses were conducted using SPSS version 20. For all models (see Table 7), four steps were carried out. In the first step for all models, binary logistic regressions were conducted for each set of variables: (1) demographic factors (APOE included), (2) neurocognitive test scores, (3) biomarkers obtained from CSF, blood, and urine, and (4) MRI and PET imaging. Factors meeting a more liberal significance threshold of $\leq 0.1$ for each class of variables were retained for the second step. For the "hand selected" model, the second step consisted of factors being entered into a second binary logistic regression and then removed until an unsatisfactory reduction in Nagelkerke (or pseudo-R ${ }^{2}$ ) was noted. For all other models, the second step consisted of remaining factors from each group being entered in a backward elimination binary logistic regression. For the third step, scores for each patient were calculated using the resulting regression equation (i.e., (constant) $+($ variable score* $\beta))$. Using the resulting scores on the regression equation, receiving operating characteristic plots (ROC) were calculated for each model. Using ROC plots, sensitivity and specificity for each subject was obtained by taking sensitivity against 1-specificity (see Altman \& Bland, 1994 for a detailed description of ROC plots). For each model, the variable score is represented by the patient's performance on that variable (e.g., Logical Memory II score, hippocampus volume, etc.). These scores are then multiplied by the unstandardized beta weight for each variable obtained from conducting the regression. Next, these values and the constant of the regression are summed into a single value that constitutes the predicted 
score. This score is then compared to the optimal cut-score that was obtained from the ROC analysis of the distribution of all predicted scores. Next, the psychometric properties corresponding to each equation (i.e., sensitivity, specificity, negative predictive value, and positive predictive value) can be applied. Finally, a probability can be given for the likelihood of converting to dementia.

A separate set of binary logistic regressions were also conducted to determine the association between each resulting model to the diagnostic classification system from which it was derived as well as the association across classification systems.

An independent sample $t$ test was conducted to determine if daily functioning was significantly different for converters versus nonconverters at baseline. 


\section{RESULTS}

\section{Sample}

Sample demographics for all patients identified as MCI according to all classification systems can be found in Table 8 .

Hypothesis \#1: Can demographic factors be used to predict conversion from MCI to dementia? It is hypothesized that the female gender and low education will yield the highest predictive values.

No demographic variables were able to predict conversion from MCI to dementia at the .01 level (gender, $B=.245, p=.137$; education, $B=-.008, p=.765$; race, $B=.042$, $p=.643$; age $, B<.001, p=.652)$ using ADNI diagnostic criteria.

Hypothesis \#2: Can neuropsychological testing be used to predict conversion from MCI to

dementia? It is hypothesized Logical Memory - II will yield the highest predictive value of any measure.

Results of a backwards elimination regression using existing ADNI diagnostic criteria demonstrated that neuropsychological testing can be used to predict conversion from MCI to dementia with modest accuracy (pseudo $R^{2}=.241, \mathrm{ROC}=.823$ ). Using the optimal cutting score of -2.32 , sensitivity of .80, and specificity of .71 was observed. For lower equation scores, sensitivity increased at the cost of specificity. For higher equation scores, specificity increased at the cost of sensitivity. Eleven variables were 
retained in the final equation based on neurocognitive performance, and four were significant predictors at the .01 level (Table 9). As expected, Logical Memory - II yielded the highest predictive value of any measure comprising the test battery $(\beta=-.177$, $p<.001)$.

Hypothesis \#3: Can biomarkers collected from CSF, urine, or blood be used to predict conversion from MCI to dementia? It is hypothesized that homocysteine levels will yield the highest predictive value.

Results of a binary logistic regression show the only biomarker collected from CSF, urine, or blood to be a significant predictor of conversion was level of amyloid-beta (Abeta) 40 in plasma $(\beta=-.017, p=.003)$ using ADNI diagnostic criteria. Homocysteine was not a significant predictor of conversion $(\beta=.014, p=.824)$. The following biomarkers collected from CSF, urine or blood were also not significant predictors: 8-isoPGF2alpha isoprostane $(\beta=.024, p=.814) ; 8,12$-iso-iPF2alpha isoprostane $(\beta=.054$, $p=.102)$; alpha-synuclein $(\beta=.138, p=.681)$; CSF Tau level $(\beta=-.007, p=.322)$; Abeta1-42 $(\beta=-.005, p=.322 ; \mathrm{pTau181p}(\beta=.033, p=.192) ;$ and Abeta $42(\beta=.036, p=.152)$.

Hypothesis \#4: Can brain imaging be used to predict conversion from MCI to dementia? It is hypothesized hypometabolism in the hippocampal brain area will yield the highest

predictive value.

Results of a backwards elimination regression using existing ADNI diagnostic criteria demonstrated that brain imaging can be used to predict conversion from MCI to dementia with modest accuracy (pseudo $R^{2}=.371, \mathrm{ROC}=.859$ ). Using the optimal cutting score of -2.04 , predicted values generated by the equation achieved sensitivity of .85 and 
specificity of .71. For lower equation scores, sensitivity increased at the cost of specificity. For higher scores, specificity increased at the cost of sensitivity. Eleven variables were retained in the final equation based on brain imaging and seven were significant predictors at the .01 level (Table 10). Cumulative temporal lobe atrophy yielded the highest predictive value of any imaging variable $(\beta=-.063, p<.001)$.

Using ADNI criteria, the only PET variable that was a significant predictor at the .01 level was globally normalized cerebral metabolic rate for glucose (CMRgl) in the left mid temporal gyrus $(\beta=-5.936, p=.01)$. No PET variables were retained in the final imaging model.

In terms of laterality, remarkable consistency was found in the final neuroimaging model. For the right hemisphere, five brain structures were represented in the final model. For the left hemisphere, five brain structures were also represented in the final model.

Hypothesis \#5: Can ADLs and IADLs be used to predict conversion from MCI to dementia? It is hypothesized that performance on tests of neuropsychological functioning will be a better predictor of conversion than ADL performance.

FAQ total score $(\beta=.123, p<.001)$ was a significant predictor at the .01 level using ADNI diagnostic criteria. However, functional abilities were not retained in the final model, whereas, measures of delayed episodic memory were. FAQ scores were significantly poorer for converters (mean 7.63, SD 5.6, range 0-28) than for nonconverters (mean 3.91, SD 4.6, range $0-27)$ at baseline $(t[392]=-5.687, p<0.001)$.

Hypothesis \#6: Can neuropsychiatric functioning be used to predict conversion from MCI to dementia? It is hypothesized depression will yield the highest predictive value of any behavioral abnormality. 
Using ADNI criteria, no neuropsychiatric variable was a significant predictor at the .01 level: $\operatorname{GDS}(\beta=.205, p=.475)$ and NPI $(\beta=.042, p=.800)$.

Hypothesis \#7: Can data from neuropsychological testing, demographic variables, biomarkers, brain imaging, ADLs, and neuropsychiatric functioning be used to construct a statistical model that predicts conversion from MCI to dementia with a high level of accuracy? Furthermore, can this model be used to predict conversion from MCI to dementia better than predictions made from any single variable or variables from any factor groups (i.e., neuropsychological testing, demographic variables, biomarkers, and brain imaging)?

In total, 13 variables (see Table 11) were retained in the final model (pseudo R2=.61, $\mathrm{ROC}=.93$ ) using the ADNI hand selected (see Statistical Analyses section) method (ADNI hand selected model). A large proportion of variance was accounted for by the equation, and area under the curve was good. Optimal cutting scores for predicted values generated by the equation achieved sensitivity of .94 and specificity of .79 for a positive predictive value of .66 and negative predictive value of .99. For lower equation scores, sensitivity increased at the cost of specificity. For higher equation scores, specificity increased at the cost of sensitivity.

Hypothesis \#8: What diagnostic criteria can be used to construct an optimal screening model?

For example, does a 1.0 or 1.5 SD cutoff using age-and education-adjusted scores on tests of neuropsychological functioning yield more acceptable inclusion rates in application of the screening model? Also, does using premorbid intelligence increase the psychometric properties of a screening model? Finally, does allowing for some 
minor difficulties with ADLs yield a more optimal screening model? It is hypothesized that using a 1.0 SD cutoff will be preferable to using a 1.5 SD cutoff, that taking premorbid intelligence into account will increase the psychometric properties of the screening model, and that allowing for some minor trouble with ADLs will further increase the psychometric properties of a screening model.

The number and percentage of patients identified as normal, MCI, and mild $\mathrm{AD}$ using each classification system can be found in Table 12. For each classification system, statistical models were constructed to predict conversion from MCI to dementia (see Table 7 for a detailed description of diagnostic criteria used for each classification system). Two models were constructed for ADNI criteria using different methods: a backward elimination method (ADNI backward elimination model) and a hand selected method (ADNI hand selected model) (see the statistical analyses section for a detailed description of the methods used to construct these two models). For the backward elimination model (Table 13), 11 variables were retained in the final model (pseudo $\left.R^{2}=.50, R O C=.91\right)$. For the hand selected model (Table 11), 13 variables were retained in the final model (pseudo $R^{2}=.61, R O C=.93$ ). For Model \#1 (Table 14), 23 variables were retained in the final model (pseudo $R^{2}=.74, R O C=.97$ ). For Model \#2 (Table 15), 12 variables were retained (pseudo $R^{2}=.77, R O C=.97$ ) in the final model. For Model \#3 (Table 16), 6 variables were retained (pseudo $R^{2}=.41, R O C=.88$ ) in the final model.

Cutting scores for predicted values generated by each equation with sensitivity of .90 were associated with specificity of .80 for the ADNI hand selected model, specificity of .71 for the ADNI backward elimination model, specificity of .90 for proposed model 
$\# 1$, specificity of .95 for proposed model \#2, and specificity of .76 for proposed model \#3.

Final equations for each model were compared across each diagnostic system (see Tables 17 and 18). For example, how well does the ADNI hand selected model perform for patients identified as MCI using proposed criteria \#1, and so on. Table 17 lists the results of a series of binary logistic regressions. As can be seen, with the exception of the ADNI backwards elimination model, each resulting model score was a significant predictor of conversion using the associated classification system. For example, the ADNI hand selected model was a significant predictor of conversion using ADNI diagnostic criteria, model \#1 was a significant predictor of conversion using proposed criteria $\# 1$, model $\# 2$ was a significant predictor of conversion using proposed criteria $\# 2$, and model \#3 was a significant predictor of conversion using proposed criteria \#3. The only model that was a significant predictor at the .01 level across classification systems was Model \#1. It was a significant predictor using ADNI diagnostic criteria $(\beta=6.032$, $p<.001)$ and using proposed criteria $\# 3(\beta=3.558, p=.002)$. Model $\# 1$ trended towards significance using proposed criteria \#2 ( $\beta=9.190, p=.051)$. In Table 18, pseudo $R^{2}$ and AUC was examined across diagnostic system. Similar to the results listed in Table 17, Model \#1 appears to be the best performing model across diagnostic classification systems.

When only allowing for "essentially normal" ADLs (defined as $0-1$ on the FAQ), 108 patients were classified as MCI at baseline. Of these patients, 35 converted to dementia. This resulted in an unacceptably restrictive model. 


\section{Follow-Up Analyses:}

Evaluating CSF Homocysteine at the Univariate Level

Homocysteine CSF level was not predictive of conversion from MCI to dementia $(\beta=.041, p=0.134)$.

Constructing the Most User-Friendly Equation for Neuropsychologists

For this model, only raw scores for major neurocognitive indices (e.g., AVLT Total, BNT Total, etc.) and daily functioning were used. In total, six variables were retained in the final model (see Table 19). A pseudo R2 of .22 and ROC of .81 were observed. The optimal cutting score of -3.32 achieved sensitivity of .82 and specificity of .68. For lower equation scores, sensitivity increased at the cost of specificity. For higher equation scores, specificity increased at the cost of sensitivity. 


\section{DISCUSSION}

In the current study, diagnostic criteria for MCI and the ability of factors from several categories (i.e., demographic, neurocognitive, functioning, imaging, biomarker, and behavioral) to predict conversion from MCI to dementia were examined. Although having been significantly transformed since their inception, it was demonstrated through a review of the literature that current MCI diagnostic criteria are limited. First, as currently written, these criteria are reliant on human judgment. However, human judgment is subject to numerous factors that compromise its efficacy (Wedding \& Faust, 1989) and render it suboptimal to judgments based on equations derived from statistical analyses (Dawes, 2005; Dawes, Faust, \& Meehl, 1989; Garb, 1994; Goldberg, 1965; Goldberg, 1968; Goldberg, 1970; Grove et al., 2000; Marchese, 1992; Meehl, 1954; Meehl, 1984; Sines, 1971; Wiggins, 1981). Second, multiple diagnostic criteria are available so different criteria are being used by different clinicians. Third, as currently written, all available criteria are somewhat ambiguous and can be applied in different ways. For example, whereas one clinician judges impairment to be $1.5 \mathrm{SD}$ below the mean, another clinician may judge impairment to be $1.0 \mathrm{SD}$ below the mean.

Consequently, error variance is introduced and has led to widely varying estimates of incidence, prevalence, and conversion.

Although eight hypotheses were explored in the current study, each of the first six hypotheses were constructed to lead up to the final two overarching hypotheses: MCI 
diagnostic criteria can be modified in a manner that removes much of the necessity of using human judgment and a statistical equation can be constructed to predict conversion from MCI to dementia with a high degree of accuracy. Results of the current study supported these two hypotheses. First, it was demonstrated that MCI diagnostic criteria could be modified in a manner that lessens the reliance on human judgment but also enhances the ease with which MCI diagnostic criteria can be utilized and applied. Second, it was demonstrated that an equation could be constructed using regression modeling that predicts conversion from MCI to dementia with a high degree of accuracy. Although this finding was consistent across varying applications of diagnostic criteria, the model that demonstrated the best level of predictive power was the model using (1) intact general cognitive functioning, (2) a 1.0 SD cutoff on one or more cognitive domains, and (3) an allowance for some level of functional impairment. This finding was consistent with a priori expectations.

For MCI to truly represent its conceptual base—as a predementia syndrome with an etiology commensurate to dementia - the diagnostic criteria should be constructed in such a way that a high number of people diagnosed with MCI go on to develop dementia. In this paper, an attempt was made to redefine the diagnostic criteria currently available for MCI. However, the two overarching hypotheses are composed of variables representing a number of different classes (i.e., demographic, neurocognitive, functional, imaging, biomarker, and behavioral variables), and each of these parts is represented by the other six research hypotheses. In the sections that follow, each of these parts (i.e., "building-block" hypotheses) will be discussed with rationale pertaining to the reasoning behind the hypothesis formulation. In ADL and IADL performance, for example, a priori 
expectations stated that both a screening and conversion model would be aided by aspects of functional ability. Thus, daily functioning was expected to be a variable with a unique contribution to conversion when examined in isolation from variables in other categories and also to combine with variables from other categories to make an effective prediction model. Thus, these parts or "building-block" hypotheses (e.g., functional ability) were analyzed in a step-by-step fashion and used to build-up to the two overarching hypotheses. Without including these "building-block" steps as a point of reference, the main points (i.e., overarching hypotheses) could not be fully discussed. In the sections that follow, each of the eight hypotheses evaluated in this paper will be discussed. The final two hypotheses reflecting the two main points of this study will be discussed in terms of their contributing parts or building-blocks. A discussion of the limitations of this study and potential future directions of research will follow. Finally, clinical and research implications of the findings outlined in this study will be discussed and followed by a brief conclusion.

Hypothesis \#1: Can demographic factors be used to predict conversion from MCI to dementia? It is hypothesized that the female gender and low education will yield the highest predictive values.

\section{Education}

It was expected in the current study that education would serve as a protective factor from conversion. Contrary to this expectation, however, education was not observed to protect a person with MCI from converting to dementia. These results seem to indicate that once domain specific impairment is present education no longer functions as a protective factor. These results also indicate that with a threshold of cognitive 
impairment education no longer protects against future general cognitive impairment and/or functional impairment. One of two explanations seems likely. First, once a person is impaired in at least one cognitive domain, the mitigating aspects of a cognitive reserve are all but extinguished. Thus, domain specific impairment renders the protective qualities of education inert regardless of level of education. Second, to be classified as exhibiting domain specific impairment, a person with a high level of education is already more impaired than a person with a low level of education and already utilizing considerable cognitive reserve to not be considered demented. Thus, whatever higher level of reserve was present has now been exhausted or almost exhausted. Exploring the validity of these two explanations was beyond the scope of this study. However, the incorporation of previous research results into the framework offered by the current study may offer some clues.

The bulk of studies available in the literature examine the protective factors of education as they relate to a healthy older adult eventually developing dementia (Brayne et al., 2010; Del Ser et al., 1999; Letenneur et al., 1999; Ott et al., 1995; Stern et al., 1994; Valenzuela et al., 2008; Valenzuela and Sachdev, 2006), but conversion is not development. For development studies, a person is recruited as a healthy older adult and followed until he/she is diagnosed with dementia. In this scenario, an unbroken timeline is present from relative good cognitive health to cognitive and functional impairment characteristic of dementia. Thus, without a point of reference in the timeline, education could be functioning as a protective factor from any aspect of dementia including domain specific cognitive impairment, general cognitive impairment, functional decline, or all three. For conversion, on the other hand, some level of domain specific cognitive 
impairment has already occurred, thereby, inserting a reference point in the timeline of normal cognition to development of dementia. Thus, only general cognitive impairment and functional decline are left as possible factors from which education could serve as a protective factor.

In contrast to the finding outlined above that states education does not protect a person with MCI from converting to dementia, the results of numerous longitudinal studies have indicated that education is a protective factor against developing dementia (Letenneur et al., 1999; Ott et al., 1995; Stern et al., 1994). The exact mechanism for this protection is unknown and two different hypotheses have been proposed. In one hypothesis, education is thought to protect the brain from the underlying pathologies associated with dementia (Del Ser et al., 1999). In the alternate hypothesis, people with more education are thought to have a better capacity to compensate for the neuropathic changes associated with dementia (Valenzuela et al., 2008; Valenzuela and Sachdev, 2006). In other words, people with more education will have brain atrophy comparable to people with less education but will be better able to compensate for this atrophy because of a greater cognitive reserve.

In an attempt to better characterize the mechanism that education has on dementia acquisition, Brayne and colleagues (2010) examined the association between education and brain pathology at death. Results indicated that although associated with decreased dementia risk and greater brain weight, education was not associated with a decrease in neuropathology. Thus, education did not protect people from atrophy but did mitigate the clinical expression of dementia. Regardless of the mechanism of protection, the more education a person had the less likely he/she was to develop dementia. However, in 
contrast to expectations, the results of the current study indicated that this trend does not hold for people already exhibiting cognitive impairment characteristic of MCI, thereby, supporting the notion that domain specific impairment exhausts the protective aspects of education.

\section{Gender}

Although controversy exists about overall gender difference in dementia development, rates of $\mathrm{AD}$ are higher among females than among males at some point in the aging process. Given that aMCI patients were included in the ADNI database combined with knowledge that women have a greater risk of developing AD, it was hypothesized that females would convert at a higher rate than males. However, this assumption was not supported by the results of the current study, and rates of conversion did not differ significantly between females and males. The likely explanations for this finding are identical to the reasons given for the lack of an effect of education. Namely, the point of interest in the timeline is actually mild cognitive impairment as opposed to dementia. However, sample specific factors may also be contributing which include age of the sample (mean of 75 years) and the inclusion of almost twice as many males than females.

In regards to any dementia type, previous studies on incidence rates in males and females are mixed. Results of some studies indicate that females are more likely to develop dementia than males with gender differences becoming more pronounced for people over the age of 85 (Anderson et al., 1999; Brayne et al., 1995; Clark et al., 1996; Fratiglioni et al., 1997; Morgan et al., 1993; Yoshitake et al., 1995). But results of other studies have noted a lack of significantly different incidence rates of dementia between 
females and males (Bachman et al., 1993; Ganguli et al., 2000; Letenneur et al., 1994; Paykel et al., 1994; Rocca et al., 1998). In contrast to conversion to any dementia type, the focus of the current study was conversion specifically to AD. In the AD literature, results consistently note that development becomes more prevalent in females than in males at some point during the aging process (Brayne et al., 1995; Fratiglioni et al., 1997; Hagnell, Ojesjo, \& Rorsman, 1992; Letenneur et al., 1994). Thus, it was expected that this trend would extend from development to conversion. However, as noted above, this expectation was not found.

Hypothesis \#2: Can neuropsychological testing be used to predict conversion from MCI to

dementia? It is hypothesized Logical Memory - II will yield the highest predictive value of any measure.

In the current study, an evaluation of neurocognitive results indicated that four variables were significant predictors of conversion when analyzed in combination with all other neuropsychological variables (see Table 10). As can be seen, all of these variables relate to three cognitive domains: (delayed) memory, language, and processing speed. Next, using only neuropsychological variables, an equation was constructed to identify people with MCI who would eventually convert to dementia. The resulting model accounted for roughly a quarter of variance. Moreover, its associated clinical cutoff may provide clinicians with a useful tool when questions about conversion arise. Using only a person's neurocognitive profile, the resulting equation predicted conversion from MCI to AD with modest accuracy. As expected, a test of delayed episodic memory (i.e., Logical Memory - II) exhibited the highest predictive power of any 
neuropsychological variable. Other measures of episodic memory as well as measures of recognition memory, executive functioning, language, attention, and processing speed were also retained in the final model (Table 9). The optimal cut-score for this equation was noted as obtaining sensitivity of $80 \%$ and specificity of $71 \%$. The equation along with examples of using the equation can be found in Table 9 and Appendix \#1. In addition, an Excel spreadsheet and detailed instructions for working this equation can be obtained from the first author of this study.

Like in the current study, the association between conversion from MCI to AD and neuropsychological variables has previously been examined. Consistent with the results of this study, results of prior studies show that neurocognitive functioning is a significant predictor and that memory impairment is the most powerful univariate predictor of conversion from MCI to AD (Aggarwal et al., 2005; Blacker et al., 2007; Devanand et al., 2008; Gomar et al., 2011; Tabert et al., 2006; Tierney et al., 1996). Impairments in executive functioning (Blacker et al., 2007; Gomar et al., 2011; Tabert et al., 2006; Tierney et al., 1996) and processing speed (Aggarwal et al., 2005; Gomar et al., 2011) have also been shown to significantly increase conversion risk. These results fit well with the results outlined in the current study. In addition, older adults with impaired memory as well as impaired functioning in at least one other cognitive domain are shown to be more at risk of conversion over a three-year period (Tabert et al., 2006). These findings also assimilate well with the results of the current study which demonstrated that impairment in three cognitive domains (i.e., episodic memory, language, and processing speed) was particularly predictive of conversion over a one-year period. However, the 
model and resulting equation offered in the current work expand upon these findings and present the opportunity for enhanced clinical utility.

Regression models have been detailed prior to the current study. Moreover, like in the current study, regression models have been utilized to determine the ability of numerous neuropsychological variables to predict conversion. In a sample of 218 older adults diagnosed with MCI, measures of episodic memory, semantic memory, working memory, and processing speed were all noted as univariate predictors of conversion (Aggarwal et al., 2005) with performance on a measure of visuospatial ability not being associated with conversion. When all variables were analyzed together, only episodic memory and processing speed remained significant predictors with episodic memory the strongest predictor at both the univariate and multivariate level. Older adults with poorer episodic memory were twice as likely to convert when compared with older adults who have diminished performance in another cognitive domain. Moreover, poorer baseline episodic memory was predictive of a more rapid overall decline and an increased risk of conversion over a 10-year period. A decline in a cognitive domain other than memory was only predictive of conversion for the year immediately following onset of singledomain impairment. Executive functioning (Blacker et al., 2007; Tabert et al., 2007; Tierney et al., 1996) has also been observed to significantly predict conversion and when combined with performance on a measure of delayed visual memory has been noted as achieving sensitivity of $76 \%$ and specificity of $94 \%$ (Tierney et al., 1996). Although these findings add to the research literature and are useful in a clinical context, the ability of clinicians to apply this information is limited. Thus, the detailing of a statistical model 
in the present work offers an exciting expansion of regression models for the prediction of conversion from MCI to dementia.

Hypothesis \#3: Can biomarkers collected from CSF, urine, or blood be used to predict conversion from MCI to dementia? It is hypothesized that homocysteine levels will yield the highest predictive value.

The term "biomarker" refers to entities that are associated with a disease through concentration, presence, and activity (Anoop, Singh, Jacob \& Maji, 2010). In the current study, the only biomarker collected from CSF, urine, and blood/plasma to be a significant predictor of conversion was $A \beta 40$. A $\beta$ or amyloid-beta is a term used to describe peptides of 36-43 amino acids. A $\beta 40$ has been shown to be present in both senile plaques as well as vascular amyloid and both senile plaques and vascular amyloid are hallmarks of dementia (Castano et al., 1996). Therefore, given the association senile plaques and vascular amyloid have with dementia and AD (Castano et al., 1996), it is not surprising that some form of amyloid-beta was able to predict conversion from aMCI to AD.

In contrast to a priori expectations, homocysteine CSF level was not significantly related to conversion. A potential reason that a discrepancy exists between the findings of the current study and those noted by Mattsson and colleagues (2009) is that AD biomarkers in the latter study were examined at the univariate level. Thus, homocysteine CSF level was analyzed at the univariate level for a follow-up analysis in the current study. Once again, findings were contrary to those by Mattsson and colleagues (2009), and homocysteine CSF levels were not predictive of conversion even when analyzed in isolation. As noted by Jack et al. (2010) a possible reason for the above mentioned discrepancy may be one of timing because different biomarkers may have different 
predictive values at varying times in the progression of a disease. For example, in MCI, progression during the first year may be highly associated with baseline markers of neurodegeneration. However, progression may be better accounted for by markers of brain amyloid over a longer longitudinal course (Dickerson \& Wolk, 2013).

Earlier in this paper, a brief review of CSF, urine, and blood biomarkers in AD was conducted. As was evident in this review, most statistically relevant AD biomarkers have been discovered in CSF as opposed to urine and blood/plasma. Thus, studies have also been conducted to determine if the examination of CSF for conversion biomarkers is useful. However, most of these studies included small samples examined only at a single site. In a large-scale study of $750 \mathrm{MCI}$ patients in 12 centers located in the United States and Europe, the ability of CSF biomarkers to predict incipient AD in patients with MCI (Mattsson et al., 2009). Results indicated that three CSF biomarkers (i.e., A $\beta 42$, P-Tau, and T-Tau) could be used to predict conversion with good accuracy. Less accuracy was reported in studies utilizing smaller samples examined at a single site. The authors also noted that some of the variability in the literature is present because the procedures used for designating biomarker cutoffs as well as the cutoffs themselves varied considerably from study to study. Thus, optimal clinical cut-scores have yet to be established for biomarker data and clinical judgment appears to be an issue in the study of these variables. Regardless, given that biomarkers of conversion were noted in a large-scale study, it was expected that the current results would fundamentally match these earlier findings. However, this expectation was not met. 
Hypothesis \#4: Can brain imaging be used to predict conversion from MCI to dementia? It is hypothesized hypometabolism in the hippocampal brain area will yield the highest

predictive value.

In the current study, an evaluation of MRI results indicated that six areas were significant predictors of conversion when analyzed in combination with all other MRI variables: cumulative temporal lobe atrophy, left hippocampal volume, right precentral cortex volume, right middle orbital grey matter volume, right posterior cingulum grey matter volume, left superior temporal gyrus volume, and right cerebellum grey matter volume (see Table 10). As expected, with the exception of right cerebellum grey matter volume and right posterior cingulum volume, all of these areas are located in the frontal or temporal lobes and consistent with previous imaging studies evaluating conversion from MCI to dementia (Devanand et al., 2007; Whitwell et al., 2008).

For PET, only glucose consumption in the left middle temporal gyrus was a significant predictor of conversion when analyzed in combination with all other PET variables. However, this variable was not retained in the final imaging model. Contrary to a priori hypotheses, reduced glucose consumption in the hippocampus was not the strongest imaging predictor. In this sample, cumulative temporal lobe atrophy exhibited the greatest predictive value when compared to PET and MRI variables.

Next, an equation was constructed to identify people with $\mathrm{MCI}$ that would convert to dementia using only imaging variables. This model accounted for approximately a third of variance in conversion, and the resulting equation (see Table 10) predicted conversion from $\mathrm{MCI}$ to $\mathrm{AD}$ with modest accuracy. The optimal cut-score for this 
equation (-2.04) was noted as obtaining sensitivity of $85 \%$ and specificity of $71 \%$ via ROC analysis. The equation along with examples of using the equation can be found in Table 10 and Appendix \#2. In addition, an Excel spreadsheet and detailed instructions for working this equation can be obtained from the first author of this study.

As in the current study, the utility of using imaging results to predict conversion in MCI patients has previously been examined. In one such study, 139 people with MCI and 63 healthy controls were scanned using MRI and followed for five years (Devanand et al., 2007). Consistent with the results of the current study, temporal structures could be used to classify sample groups. At baseline, Devanand and colleagues (2007) noted that hippocampal and entorhinal cortex volumes were largest in healthy controls, intermediate in nonconverters, and smallest in converters. Relative volumes of these same brain areas were also found to significantly predict time to conversion. In another study, 63 people with aMCI and 63 demographically matched controls were scanned using MRI and followed for three years (Whitwell et al., 2008). When compared to controls, MCI patients that converted to dementia had bilateral loss affecting the medial and inferior temporal lobe, the temporoparietal association cortex, and the frontal lobes. When compared to nonconverters, MCI patients that converted to dementia had MRI profiles strikingly similar to those of the current study. Also in the study by Whitwell and colleagues (2008), greater loss was noted in the medial and inferior temporal lobes, the temporoparietal cortex, the posterior cingulate, the precuneus, the anterior cingulate, and the frontal lobes.

In contrast to MRI, FDG-PET studies in MCI are controversial because reduced cerebral metabolic rate of glucose consumption may not be detectable outside the 
hippocampal formation in patients with minimal impairment (Schuff \& Zhu, 2007). However, in a small PET study, cortical changes to glucose metabolism were observed in a group of MCI patients that were scanned twice over a year (Drzezga et al., 2003). Glucose consumption was lower in the posterior cingulate cortex and precuneus for converters than it was for nonconverters at baseline. At follow-up, glucose consumption was lower in bilateral prefrontal areas, and reduced consumption had progressed in the posterior cingulate cortex. This finding was not supported by the results of the current study since only hypometabolism in the left middle temporal gyrus was predictive of conversion at the .01 level.

\section{Hypothesis \#5: Can daily functioning be used to predict conversion from MCI to}

dementia? It is hypothesized that performance on tests of neuropsychological functioning will be a better predictor of conversion than daily functioning.

Consistent with a priori expectations, functional abilities significantly predicted conversion. Interestingly, although functional abilities were poorer for converters at baseline, nonconverters also demonstrated multiple functional decrements even when utilizing the FAQ - a means of evaluation less objective than a performance-based measure. Thus, it not only appears that functional impairment is a characteristic of MCI, but it also appears that a continuum exists between MCI and dementia in regards to functional impairment. Also consistent with prior expectations, delayed episodic memory was a stronger univariate predictor of conversion than functional ability.

Prior research has shown that although basic ADLs (i.e., eating, dressing, bathing, etc.) remain preserved in people with mild cognitive impairments, higher-order IADLs (i.e., financial and medication management, using public transportation, interacting with 
technology, etc.) are often subtly impaired (Winblad et al., 2004). But as highlighted earlier, diagnostic criteria related to everyday functioning in $\mathrm{MCI}$ are (see Tables 1 and 3) constructed in a manner that assumes delineation between virtually intact functional abilities for MCI and impaired functional abilities for dementia. However, the results of this study do not support this delineation as both converters and nonconverters presented with functional decrements. In addition, prior research also points to this delineation as problematic for a number of reasons (Goldberg et al., 2010). First, medical and psychiatric conditions that are reliably associated with cognitive changes are, in turn, also reliably associated with changes in everyday functioning (Goldberg et al., 1990; Heaton et al., 1994). Second, given that cognitive decline is associated with changes in everyday functioning, the cognitive impairments characteristic of MCI should exact a graduated cost on function (Goldberg et al., 2010).

In addition to issues with the current diagnostic criteria, the evaluation of daily functioning in $\mathrm{MCI}$ is flawed because standardized measures sensitive to minor functional changes are not commonly utilized (Goldberg et al., 2010). At present, the most common method for garnering information about the everyday functioning of a patient is to interview a close relative. However, the reliability of this method suffers because of halo effects, memory lapses, and limited knowledge that can lead to an overestimation of the functional capacity of MCI patients (Loewenstein et al., 2001). One way to increase the utility of functional evaluations would be to introduce the use of performance-based measures into standard neuropsychological test batteries. Recent research has demonstrated that patients with MCI show significantly compromised functional abilities when evaluated with performance-based measures, and this trend 
extends to patients identified as having intact functioning by family members (Goldberg et al., 2010).

Unfortunately, performance-based measures were not utilized as part of the ADNI study. However, the measure utilized in ADNI (i.e., FAQ) was designed to evaluate a number of higher-order IADLs and produce a total score that can be compared between patients. Given that patients with MCI likely demonstrate functional impairment on a continuum with dementia, it was assumed that differences would be present and statistically discernable among patients who convert to dementia and patients who do not convert to dementia. This expectation was supported by the findings of this study.

Hypothesis \#6: Can neuropsychiatric functioning be used to predict conversion from MCI to dementia? It is hypothesized that depression will yield the highest predictive value of any behavioral abnormality.

Contrary to expectations, results of the current study showed that depression was not predictive of conversion to dementia in this sample of people with MCI. Of all psychiatric conditions, depression's relationship with cognitive impairment is by far the most widely studied and discussed. Up to $50 \%$ of all people living with AD have significant symptoms of depression and up to $20 \%$ meet diagnostic criteria for major depression (Wragg \& Jeste, 1989). Although results of a meta-analysis demonstrate that depression is a risk factor for dementia (Jorm, 2001), some studies have not noted an association between depression and dementia (Becker et al., 2009; Fassbender et al., 1999; Hendersen et al., 1997). Moreover, the role of depression as a risk for dementia is not well understood, and multiple hypotheses explaining the association are found in the 
literature (Jorm, 2001). These hypotheses include: (1) depression is an early prodome of dementia, (2) depression brings forward the clinical aspects of dementia, and (3) depression leads to hippocampal damage.

The expectation that depression would be predictive of conversion was made despite its contradiction to an earlier study by Panza and colleagues (2008) that found no association between depression and conversion in a sample of 2,963 community-dwelling adults between the ages of 65-84 who were followed for 3.5 years. Moreover, results also showed that no socio-demographic or vascular risk factors could be used to modify the association between depression and conversion. However, in a more homogenous sample that utilized hospital patients, depression was able to predict conversion from MCI to dementia (Modrego and Ferrandez, 2004). The reason it was hypothesized that results of the current study would more closely match the findings of Modrego and Ferrandez (2004) than the findings of Panza et al. (2008) were twofold. First, in the 2008 study by Panza and colleagues, only $10 \%$ of the sample of people with MCI converted to dementia. Thus, it was believed that the sample likely represented a group of MCI patients more cognitively advanced than normal. Second, it was believed that the sample of hospital patients utilized by Modrego and Ferrandez (2004) would likely represent a group of people with more cognitive impairments and higher rates of depression than the people included in the ADNI sample.

As documented earlier, results of a meta-analysis noted that depression is a significant risk factor of dementia development (Jorm, 2001). Interestingly, current study findings are in contrast to this association and seem to indicate that this risk is minimized once cognitive impairment is present. The most likely reason for this dissociation is that 
studies of dementia development follow healthy controls with normal cognitive functioning while studies of conversion follow adults with some level of cognitive impairment. Thus, the current results and findings of Panza and colleagues (2008) seem to indicate that depression is a risk factor for dementia simply because it is a risk factor for cognitive impairment. In addition, these results may indicate that the presence of significant cognitive impairment dulls the additive effects of factors with lesser associations to dementia development (e.g., gender, education, depression). However, the investigation of this theory is beyond the scope of this study. Other potential reasons to explain the lack of association between conversion and depression relate to the ADNI sample itself, namely, that levels of depression were low in the ADNI sample, that the use of a self-report measure of depression lacked sensitivity, that follow-up was limited to 2-years, and that some people with depressive symptoms were receiving treatment for depressive symptoms.

Hypothesis \#7: Can data from neuropsychological testing, demographic variables, biomarkers, brain imaging, ADLs, and neuropsychiatric functioning be used to construct a statistical model that predicts conversion from MCI to dementia with a high level of accuracy? Furthermore, can this model be used to predict conversion from MCI to dementia better than predictions made from any single variable or variables from any factor groups (i.e., neuropsychological testing, demographic variables, biomarkers, and brain imaging)?

In the current study, the following variables were significant predictors of conversion when all categories of variables were taken into account (Table 11): AVLT Total Intrusions for Trial 2, BNT Total semantic cues, cumulative temporal lobe atrophy, 
right precentral grey matter, and right front mid orbital grey matter. The resulting equation accounted for approximately two-thirds of variance and predicted conversion from MCI to AD with a high level of accuracy. Moreover, this model was able to predict conversion from $\mathrm{MCI}$ to dementia better than predictions made from any single variable or variables from any factor groups. The optimal cut-score for this equation (-2.11) was noted as obtaining sensitivity of $94 \%$ and specificity of $79 \%$ for a positive predictive value of $66 \%$ and negative predictive value of $99 \%$. For people scoring above the clinical cut-score of -2.11 , the predictive value obtained from the equation indicated a high risk of converting to dementia over the course of the following year, and this was correct $66 \%$ of the time. For people scoring below or equal to -2.11 , the value obtained from the equation indicated a lower risk of conversion over the next year, and this was correct $99 \%$ of the time. Examples of this regression-based model are provided in Table 11 , and a guide for operating these types of equations can be found in the appendix. Moreover, an Excel spreadsheet and instructions can be obtained from the first author of this study that will aid in working the predictive equation.

To date, only a handful of studies have examined factors spanning a number of different categories (e.g., demographics, biomarkers, imaging, and cognition) in predicting conversion from MCI to dementia. In the first study of its kind, Jack and colleagues (1999) followed 80 patients diagnosed with MCI for an average of approximately 32 months. All 80 patients received an MRI of the head at baseline and other testing. Of the 80 patients included in the sample, 27 converted to dementia. The factor most capable of predicting conversion at the univariate level was baseline hippocampal atrophy. Moreover, the addition of age, postmenopausal estrogen 
replacement, cognition (i.e., general cognitive functioning, verbal memory, attention, and verbal fluency), APOE, history of ischemic heart disease, and hypertension did not significantly increase the estimate of relative risk. However, results of another study showed that general cognitive functioning, processing speed, and delayed verbal memory had an estimated predictive accuracy of $79 \%$ (Fleisher et al., 2007). None of hippocampal volume, ventricular volume, APOE4 genotype, and/or demographic variables improved the predictive accuracy of the model. In contrast, more recent studies have noted a combination of imaging and neuropsychological variables as possessing the best predictive validity. For example, Devanand and colleagues (2007) found that a combination of age, general cognitive functioning, delayed verbal memory, processing speed, hippocampal volume, and entorhinal cortex volume best predicted conversion from MCI to dementia. Similar findings were also noted by Gomar and colleagues (2011). In this study, only delayed verbal memory and temporal lobe cortical thickness remained significant at the multivariate level. As can be seen, later models have included both cognitive and imaging variables to achieve the greatest level of predictive accuracy, commensurate to the findings of the current study.

Hypothesis \#8: What diagnostic criteria can be used to construct an optimal screening model?

For example, does a 1.0 or 1.5 SD cutoff using age-and education-adjusted scores on tests of neuropsychological functioning yield more acceptable inclusion rates in application of the screening model? Also, does using premorbid intelligence increase the psychometric properties of a screening model? Finally, does allowing for some minor difficulties with ADLs yield a more optimal screening model? It is 
hypothesized that using a 1.0 SD cutoff will be preferable to using a 1.5 SD cutoff,

taking premorbid intelligence into account will increase the psychometric properties of the screening model, and allowing for some minor trouble with ADLs will further increase the psychometric properties of a screening model.

As can be seen in Table 12, the number of patients classified as normal were strikingly similar across diagnostic systems. Relative consistency across diagnostic system was also observed for patients classified as AD. The number of patients identified as MCI and converting to dementia (see Table 12), however, varied across diagnostic system because many patients were not able to be classified using proposed diagnostic criteria. To retain a high level of consistency among diagnostic systems, attempts were made to use ADNI criteria whenever possible. Consequently, the same cutoffs on the MMSE and CDR used for ADNI classification were used for the proposed diagnostic criteria as well. However, the introduction of a measure of daily functioning and associated cutoff caused a considerable number of patients to be unclassifiable using proposed criteria. As noted by Teng and colleagues (2010), a FAQ score $>5$ demonstrates good utility for differentiating AD from MCI. Thus, for proposed criteria, this cut-score was utilized as a diagnostic criterion. However, a subsequent issue was encountered when applying the criteria that included daily functioning because its inclusion led to discrepancies among MMSE, CDR, Logical Memory - II, and FAQ scores. For instance, using proposed criteria \#1, a patient with an MMSE of 27, CDR of 0.5, Logical Memory - II score falling more than 1.0 SD below the mean, and FAQ score of 6 would also be unclassifiable because the obtained FAQ score lies above the cutoff proposed by Teng and colleagues (2010) when all other scores lie within the MCI range 
according to ADNI criteria. It should be noted, however, when using only FAQ and Logical Memory - II scores as diagnostic criteria, almost all of the unclassified patients would have been classified as MCI, and the psychometric properties of the resulting models were very similar to those listed in Table 18.

Neurocognitive Cutoff (i.e., 1.0 vs. 1.5 SD)

The percentages of patients who converted from MCI to dementia according to each diagnostic system are listed in Table 12 . However, because of the widely varying estimates of conversion found in the literature, these percentages are not useful when comparing the efficacy of the different screening models.

Although all subjects in the current study were part of the ADNI database, the application of different diagnostic criteria essentially divided this one sample into four samples: one sample using ADNI diagnostic criteria and three samples using proposed diagnostic criteria. Thus, comparisons of performance measures (i.e., pseudo- $\mathrm{R}^{2}$ and AUC) can be made across samples/diagnostic systems (see Table 18). When evaluating the different diagnostic systems and associated models in this way, one cardinal rule must be followed: pseudo- $\mathrm{R}^{2}$ and AUC for a model should not be considered in relation to the sample from which they were derived. When evaluating the models in this manner, the model derived using Proposed Criteria \#1 (i.e., the model utilizing a 1.0 SD cutoff and allowing for scores $\leq 5$ on the FAQ) appears to have a slight advantage to the model derived using Proposed Criteria \#2 and a clear advantage to other models. In fact, the model constructed using Proposed Criteria \#1 was the best performing model whenever this general rule was followed. Taken in combination with the observation that Proposed Criteria \#1 and Proposed Criteria \#2 had the best pseudo- $\mathrm{R}^{2}$ and AUC in relation to the 
sample from which they were derived, it is clear that Proposed Criteria \#1 yielded the best screening model. Thus, it appears that a 1.0 SD cognitive cutoff is preferable to a $1.5 \mathrm{SD}$ cutoff.

\section{Daily Functioning}

One method to evaluate the usefulness of the different diagnostic systems is to compare the number of predictors used in the final conversion model. Across the five models, the number of included predictors ranged from 11 to 23 . Although still a large number of predictors, it would be easier to work with a model containing 11 predictors than it would be to work with a model containing 23 predictors. However, ease only tells a small part of the story because performance of the model in predicting conversion is much more important. Although these five models cannot be directly compared with statistical methods, statistics can be used for evaluative purposes. For example, the psychometric properties of the models in relation to their specific diagnostic criteria can be compared (see Table 18). When considering pseudo- $\mathrm{R}^{2}$ and AUC as performance measures, Model $\# 1$ and Model $\# 2$ have a clear advantage and are almost equal in utility. Interestingly, both of these models were derived from diagnostic systems that took into account scores from a measure of daily functioning (i.e., FAQ). Thus, from this limited method of comparison, it appears that allowing for some decrements to daily functioning (i.e., allowing for scores $\leq 5$ on the FAQ) improves the utility of a diagnostic classification system.

\section{Premorbid Intelligence}

Contrary to a priori hypotheses, premorbid intelligence did not appear to increase the efficacy of a screening model in this cohort of people with MCI. Evidence of this 
conclusion comes from two sources. First, Proposed Criteria \#3 was the only set of diagnostic criteria that took estimates of premorbid intelligence into account. However, the model constructed using Proposed Criteria \#3 produced the poorest performance measures when considered in relation to the sample from which they were derived (see Table 18). Moreover, the model constructed using Proposed Criteria \#3 was arguably the worst performing model across the different samples. Second, evidence for the lack of utility of premorbid intelligence can be found in the individual neuropsychological predictors that comprise the different models. For each neuropsychological measure, three values were considered: raw score, normative-corrected z-score, and normativecorrected z-score in relation to estimated premorbid IQ. Interestingly, not even a single neuropsychological variable that took into account estimated premorbid IQ was retained in the neuropsychological model (Table 9) or any model derived utilizing ADNI and/or proposed diagnostic criteria (Tables 11 and 13-17).

Follow-Up Analyses: Constructing the Most User-Friendly Equation for Neuropsychologists

To a clinician, the model that would be the easiest to use would be the model with the most obtainable data. For neuropsychologists, this model would be comprised of raw scores obtained from measures that can be administered during a brief neuropsychological evaluation. Thus, an equation was constructed using only daily functioning and raw scores from core indices of neuropsychological measures administered as part of the ADNI study. The resulting equation predicted conversion from MCI to AD with modest accuracy, and the optimal cut-score for this equation (3.32) was noted as obtaining sensitivity of $82 \%$ and specificity of $68 \%$ via ROC analysis. 
To increase the facility of this model, an Excel spreadsheet has been provided that will calculate the predicted score for a patient. Although an example, this spreadsheet demonstrates how prediction equations can be distributed for widespread clinical use. Directions for using the attached Excel spreadsheet are included in a separate document and are as follows:

1. Enter FAQ Score into cell A2 (highlighted in green).

2. Enter Logical Memory - II Total into cell B2 (highlighted in green).

3. Enter Clock Drawing Total Score into cell C2 (highlighted in green).

4. Enter AVLT Delayed Recall Total Score into cell D2 (highlighted in green).

5. Enter the seconds needed to complete Trails A into cell E2 (highlighted in green).

6. Enter ADAS-Cog Total Score into cell F2 (highlighted in green).

7. Predicted Score for the patient will appear in cell G1 (highlighted in red).

8. Compare the predicted score appearing in cell G1 to the cut-score of -3.32 .

9. Scores $>-3.32$ indicate an increased risk of conversion.

10. Scores $\leq-3.32$ indicate a decreased risk of conversion.

\section{Limitations and Future Directions}

Despite the considerable findings presented in the current study, there are a number of limitations and future directions to be considered. First, like most other multisite University-based studies, the current sample may not completely represent the population from which is was drawn. Patients needed to agree to participate in several hours of testing, have an MMSE $\geq 24$, and not have other central nervous system disorders. This in all probability yields a select MCI group, and these results might not generalize to all patients with MCI. Moreover, ADNI subjects represented a highly 
educated, Caucasian cohort of older adults with MCI. Thus, the resulting models highlighted in this paper may not translate well to other races and groups with less education. Second, for people to be included in this study sample, a subjective memory complaint was required. However, subjective memory complaints are a poor indicator of actual memory impairment (Reid \& Maclullich, 2006) and may exclude some people with MCI. Thus, future studies should not require a subjective memory complaint for inclusion. Third, a score of $\geq 24$ on the MMSE was required for study inclusion; however, the MMSE has a number of limitations that include age and education bias, limited focus on memory, and lack of an executive functioning component (Lorentz et al., 2002; Kahokehr et al., 2004; Brodaty et al., 2006). Fourth, as previously discussed, the assessment of functional decline in MCI is flawed. Although a measure evaluating several aspects of higher-order functional skills was utilized in the current study, performance-based measures of daily functioning are superior and should be included in future studies. Fifth, a large number of variables were considered when developing the models designed to predict conversion from MCI to dementia. Because of the number of variables, multiple steps had to be conducted prior to developing a final model for each diagnostic system. Future studies should limit the number of variables to ensure that the best predictors are included in conversion models. For example, since a dearth of zscores were retained in final models, the number of neuropsychological variables can be reduced by only considering raw scores. In relation to imaging, no PET variables were retained in a final model and a preponderance of retained imaging variables related to frontotemporal structures. Thus, imaging variables can be significantly reduced by limiting consideration to MRI results of frontotemporal structures. Sixth, although steps 
were taken to cross validate the models outlined in the current study, cross validation with independent studies is clearly needed. Moreover, clinical validation is necessary before conversion models can be used in clinical decision-making. Seventh, the only type of MCI considered in the current study was aMCI. However, other MCI types should be examined in future studies to investigate the utility of these findings in other MCI types as well as to develop models for MCI types other than aMCI. Eighth, conversion was defined as progressing from MCI to dementia. However, in the current study, dementia was defined according to diagnostic criteria for AD. Future studies should evaluate conversion for other dementia types including both vascular dementia and frontotemporal dementia. Also, pathological confirmation is needed to ensure that patients classified as converting from MCI to AD did not convert to a dementia type other than AD. Finally, future studies should compare clinical judgment to the equations outlined in this study.

\section{Implications}

To date, although studies have been conducted that are useful to clinicians, no studies have provided clinicians working with people with MCI a viable tool to predict which patients will convert to dementia. As a clinician, it is useful to know that a patient with MCI exhibited impairment on tests of episodic memory and that MRI results indicated mild diffuse cerebral atrophy because research has demonstrated that this patient has an increased risk for progressive decline. However, albeit useful, this information leaves a clinician in a precarious position. What should the patient and his/her family be told when questions about conversion occur? At present, the clinician can only answer these questions in vague terms. 
However, in the current study, an equation was constructed that can be applied in clinical practice. This model and associated cut-score offers neuropsychologists, as well as other health providers, an exciting opportunity. With this equation, a neuropsychologist has the potential to answer questions about conversion with much more certainty. Instead of giving vague answers, concrete probabilities based upon empirical research can be provided. Moreover, regression based models and associated equations have the potential to enhance the referral base of neuropsychologists and expand their role on medical teams. At present, typical referral questions for neuropsychologists working with older adults relate to diagnosis and differential diagnosis. However, being able to give reliable and valid probabilities of conversion rates has the potential to allow neuropsychologists the opportunity to provide geriatricians, neurologists, and general practitioners a power tool when formulating medication and treatment regimens for older adults. In regards to treatment, equations like the one presented in this paper can help identify people with MCI due to AD versus MCI due to a reversible condition. Therefore, treatments can be better personalized based on the likelihood of a person's developing dementia.

Beyond simply enhancing the usefulness of a neuropsychologist, studies of this type have the potential to change the field of neuropsychology. At present, the decisionmaking process employed by neuropsychologists has several steps. First, medical records are examined for the referral question and factors of interest. Second, the patient and family are interviewed so a detailed medical and cognitive history can be obtained with emphasis given to the onset and progression of cognitive and movement related symptoms. Third, a test battery of varying length is chosen and administered. Fourth, 
scores on the measures comprising the battery are calculated, and performance is considered in relation to demographically-matched peers. Fifth, these results are considered in relation to findings garnered from the records' review and interviews that the neuropsychologist deems important. Seventh, the neuropsychologist conducts a sometimes lengthy and exhaustive literature review in an attempt to compare the patient's profile in relation to empirical research. Finally, a diagnostic impression is rendered.

If this process appears inefficient and suboptimal, that is because it is. Further, this process relies heavily on clinical judgment and places a clinician in the position to violate numerous decision-making contraindications (e.g., overreliance on salient data, human cognitive limitations, etc.). However, with regression models, many steps in this process can be excluded, and the reliability and validity of diagnostic impressions standardized and greatly enhanced. In addition, regression based models have the potential to show which measures add incremental validity. Thus, test batteries can be streamlined and tailored for possible differential diagnoses that were hypothesized (e.g., $\mathrm{AD}$ versus frontotemporal dementia versus vascular dementia) based upon the referral question and records' review. This likely means that test batteries can be shortened, placing less stress and discomfort on the older adult patient and allowing the neuropsychologist to see more patients. Finally, quantitative representations of brain imaging should be utilized in clinical practice. At present, MRI results are interpreted based upon subjective visual impressions of MRI images usually by a radiologist or neurologist. In contrast, this method of interpretation is not utilized in research setting. Instead, quantitative analyses of imaging results are conducted. Moreover, computer programs that yield quantitative analyses of MRI data are not readily available outside of 
research settings, and clinicians are not receiving training on how to operate such programs. For MRI models to be used, quantitative MRI data will be needed. This step in the diagnostic process is important because this study has shown that a combination of imaging and cognitive variables possess the greatest potential for increasing diagnostic accuracy as it relates to MCI and dementia. Clearly, more widespread use of quantitative imaging data in clinical practice is needed.

\section{Conclusion}

At present, the diagnostic criteria for MCI are varied and able to be interpreted and implemented in different ways depending on the judgment of the clinician. Because of this issue, a wide range of incidence, prevalence, and conversion rates are found in the research literature. At its conceptual core, $\mathrm{MCI}$ is a term that was designed to describe people in the earliest stages of cognitive decline secondary to dementia. Because of issues inherent in these criteria, people with a wide range of non-dementing illnesses are being diagnosed with a predementia syndrome. Thus, many people diagnosed with MCI will have cognition and functional abilities that stay the same or even improve. In the current study, it was proposed that the best way to improve MCI diagnostic criteria is to put more of an emphasis on conversion to dementia. After all, if a person does not convert to dementia, a diagnosis of a predementia syndrome seems rather unnecessary. Moreover, by putting more of an emphasis on conversion when formulating diagnostic criteria, questions raised by the patient and his/her family that relate to conversion probabilities can be better answered.

In the current study, a model was constructed using MRI and cognitive variables that was able to predict conversion from aMCI to AD with a high degree of accuracy (see 
Table 11). Using the equation derived from the model and its corresponding cut-score, a patient could be told with $66 \%$ accuracy that he/she would convert to dementia within the next year or with $99 \%$ accuracy that he/she would not convert to dementia within the next year. The cut-score was selected to optimize sensitivity and identify a large majority of people who would convert. However, different cutoffs could be selected for different purposes, thereby, increasing the accuracy of a positive test result. Although equations like this have the potential to drastically improve the way clinicians diagnose dementing diseases, a number of issues concerning their application was illustrated in this paper (e.g., quantitative MRI data is not readily available). Thus, a simple user-friendly model was constructed that utilizes only information that can be obtained during a brief evaluation (see Table 19). The resulting model was used to predict conversion with modest accuracy. In addition, this model was also used to demonstrate the ease of use of such an equation if an Excel spreadsheet and directions are included. Finally, a number of other models were constructed to test the utility of different diagnostic systems. As expected, the classification system with the best predictive validity utilized a cognitive cutoff of 1.0 SD on Logical Memory - II and allowed for some level of functional decline (i.e., scores $\leq 5$ on the FAQ). The equation derived from this model included variables representing functional ability, neurocognitive performance, and MRI volumetric measures. In summary, these results clearly indicate that MCI diagnostic criteria need to be better standardized, so clinical judgment can be taken out of the equation. 
Table 1. MCI Diagnostic Criteria and Current Recommendations

\section{Petersen Criteria}

(Petersen et al., 1997)

\section{MCI-R Criteria}

(Winblad et al., 2004)
1. Presence of a subjective memory complaint

2. Preserved general intellectual functioning

3. Demonstration of a memory impairment by cognitive testing

4. Intact ability to perform ADLs

5. Absence of dementia
1. Presence of a cognitive complaint from either the patient and/or family member

2. Absence of dementia

3. Change from normal functioning

4. Decline in any area of cognitive functioning 5. Preserved overall general functioning but possibly with increasing difficulty in the performance of ADLs

\footnotetext{
Note. MCI-R=mild cognitive impairment-revised
} 
Table 2. MCI-R Subtype and Etiology (Winblad et al., 2004.)

\begin{tabular}{lc}
\hline MCI-R Subtype & Etiology \\
\hline amnestic-MCI (aMCI) (single & degenerative, vascular, metabolic, traumatic, \\
and multiple domains) & psychiatric, other \\
multiple domains, slightly & degenerative, vascular, metabolic, traumatic, \\
impaired-MCI (mdMCI) & psychiatric, other \\
single nonmemory domain- & degenerative, vascular, metabolic, traumatic, \\
MCI (snMCI) & psychiatric, other
\end{tabular}

Note. $\mathrm{MCI}=$ mild cognitive impairment, $\mathrm{MCI}-\mathrm{R}=$ mild cognitive impairment-revised 
Table 3. Recommendations from the Alzheimer's Group (Albert et al., 2011)

1. Cognitive concern reflecting a change in cognition reported by the patient or informant or clinician (i.e., historical or observed evidence of decline over time)

2. Objective evidence of impairment in one or more cognitive domains, typically including memory (i.e., formal or bedside testing to establish level of cognitive function in multiple domains)

3. Preservation of independence in functional abilities

4. Intact ability to perform ADLs

5. Not demented

6. Provide evidence of longitudinal decline in cognition, when feasible

Note. $\mathrm{ADL}=$ activities of daily living. 
Table 4. Biomarkers Under Examination for AD (Albert et al., 2011)

\section{Biomarkers of A $\boldsymbol{\beta}$ deposition}

CSF A $\beta 42$

PET amyloid imaging

\section{Biomarkers of neuronal injury}

CSF tau/phosphorylated-tau

Hippocampal volume or medial temporal atrophy by volumetric measures

or visual rating

Rate of brain atrophy

FDG-PET imaging

SPECT perfusion imaging

Less well validated biomarkers: fMRI activation studies, resting BOLD

functional connectivity, MRI perfusion, MR spectroscopy, diffusion

tensor imaging, voxel-based and multivariate measures

\section{Associated biochemical change}

Inflammatory biomarkers (cytokines)

Oxidative stress (isoprostanes)

Other markers of synaptic damage and neurodegeneration such as cell death

Note. $\mathrm{AD}=\mathrm{Alzheimer}$ 's disease, $\mathrm{A} \beta=$ beta-amyloid protein, $\mathrm{CSF}=$ cerebrospinal fluid, $\mathrm{PET}=$ positron emission tomography, $\mathrm{FDG}=$ fluorodeoxyglucose, $\mathrm{SPECT}=$ single photon emission tomography, $\mathrm{MRI}=$ magnetic resonance imaging, $\mathrm{fMRI}=$ functional magnetic resonance imaging, $\mathrm{BOLD}=$ blood oxygen level-dependent, $\mathrm{MR}=$ magnetic resonance. 
Table 5. MCI Criteria Incorporating Biomarkers (Albert et al., 2011)

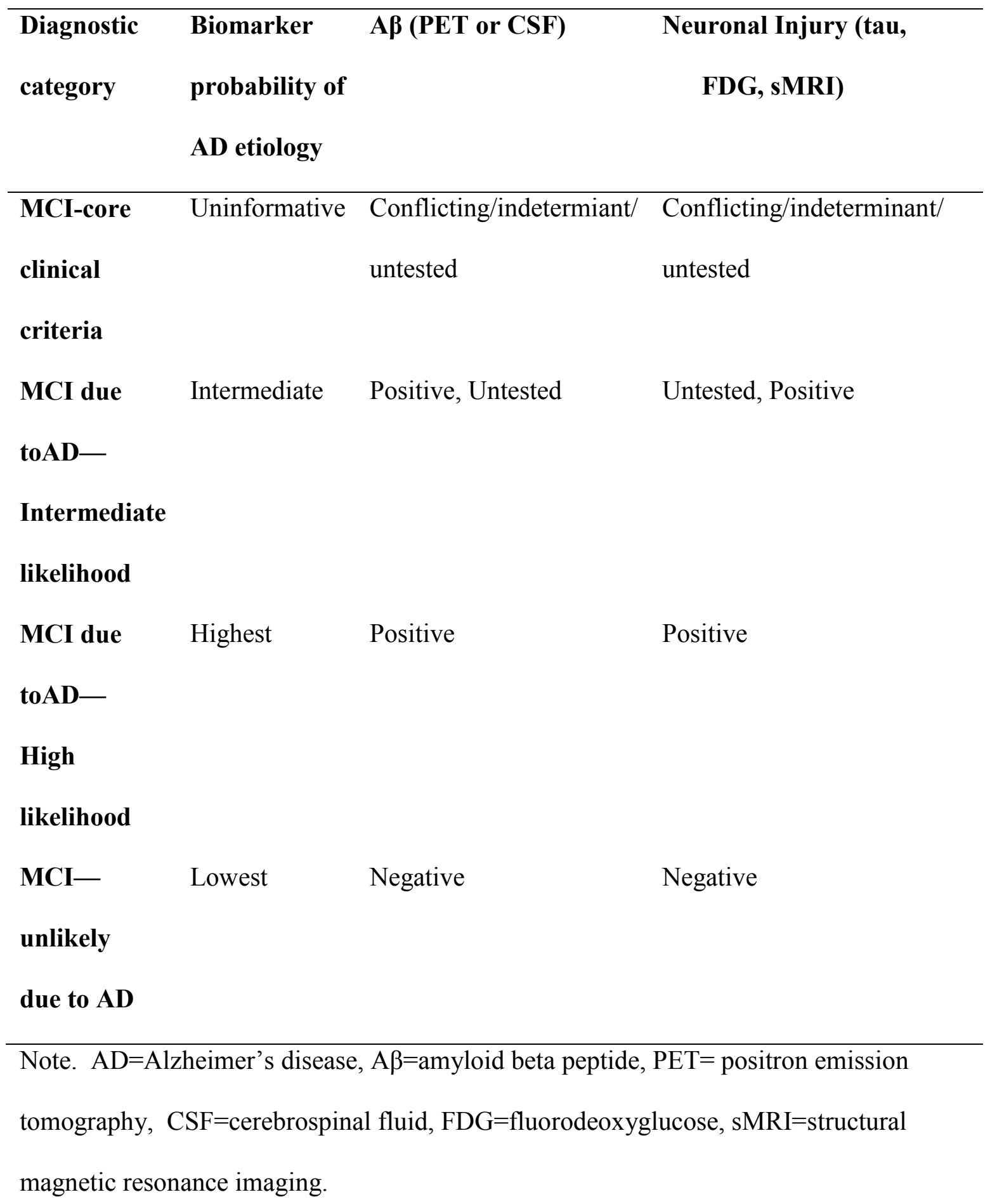


Table 6. Current Recommendations for Standardization of MCI Diagnostic Criteria

1. Intact general cognitive functioning as demonstrated by performance falling greater than 1.0 SD above the mean on a test of general cognitive functioning (e.g., DRS-2)

2. Objective evidence of impairment in one or more cognitive domain as determined by performance falling at $1.0 \mathrm{SD}$ or lower below the mean and/or estimates of premorbid intelligence using age- and education-corrected scores on a neuropsychological battery (normative data corrected for race should be used whenever possible)

3. Preserved overall general functioning but possibly with increasing difficulty in the performance of ADLs as determined by a measure of functional/ADL performance (i.e., $>80 \%$ on the SE-ADL scale)

4. Not demented

Note. $\mathrm{MCI}=$ mild cognitive impairment, $\mathrm{SD}=$ standard deviation, $\mathrm{DRS}=$ Dementia Rating Scale, $\mathrm{ADL}=$ activities of daily living, $\mathrm{SE}-\mathrm{ADL}=\mathrm{Schwab} \&$ England-ADLs. 
Table 7. Diagnostic Criteria for Classification Systems.

\begin{tabular}{|c|c|c|c|c|c|}
\hline & & ADNI Criteria & Proposed & Proposed & Proposed Criteria \#3 \\
\hline & & & Criteri & Criteri & \\
\hline & & & $\mathbf{a} \# \mathbf{1}$ & $\mathbf{a} \# \mathbf{2}$ & \\
\hline CDR & Normal & 0 & 0 & 0 & 0 \\
\hline & MCI & 0.5 & 0.5 & 0.5 & 0.5 \\
\hline & Mild AD & $0.5-1.0$ & $0.5-1.0$ & $0.5-1.0$ & $0.5-1.0$ \\
\hline LM II & Normal & Above Cutoffs & $>-1.0 \mathrm{SD}$ & $>-1.5 \mathrm{SD}$ & $>-1.5$ SD from VIQ \\
\hline & MCI & Below Cutoffs & $\leq-1.0 \mathrm{SD}$ & $\leq-1.5 \mathrm{SD}$ & $\leq-1.5 \mathrm{SD}$ from VIQ \\
\hline & Mild AD & Below Cutoffs & $\leq-1.0 \mathrm{SD}$ & $\leq-1.5 \mathrm{SD}$ & $\leq-1.5 \mathrm{SD}$ from VIQ \\
\hline MMSE & Normal & $24-30$ & $24-30$ & $24-30$ & $24-30$ \\
\hline & MCI & $24-30$ & $24-30$ & $24-30$ & $24-30$ \\
\hline & Mild AD & $20-26$ & $20-26$ & $20-26$ & $20-26$ \\
\hline Memory & Normal & None & None & None & None \\
\hline Complaint & MCI & Yes & Yes & Yes & Yes \\
\hline & Mild AD & Yes & Yes & Yes & Yes \\
\hline Daily & Normal & No changes & $\leq 5$ on FAQ & $\leq 5$ on FAQ & $\leq 5$ on FAQ \\
\hline Functioning & MCI & Essentially Normal & $\leq 5$ on FAQ & $\leq 5$ on $\mathrm{FAQ}$ & $\leq 5$ on FAQ \\
\hline & Mild AD & Decline & $\geq 6$ on FAQ & $\geq 6$ on FAQ & $\geq 6$ on FAQ \\
\hline
\end{tabular}

Note. Differences between classification systems are highlighted. ADNI=Alzheimer's disease Neuroimaging Initiative, $\mathrm{CDR}=\mathrm{Clinical}$ Dementia Rating, $\mathrm{LM}=$ Logical Memory, MMSE $=$ Mini-Mental Status Examination, $\mathrm{MCI}=$ mild cognitive impairment, $\mathrm{AD}=$ Alzheimer's disease, $\mathrm{VIQ}=$ verbal intelligence quotient, $\mathrm{FAQ}=$ Functional Activities 
Questionnaire. 
Table 8. Demographics for MCI Patients by Classification System.

\begin{tabular}{lllll}
\hline & ADNI Criteria & Proposed & Proposed & Proposed \\
& $(n=397)$ & Criteria \#1 & Criteria \#2 & Criteria \#3 \\
& & $(n=191)$ & $(n=115)$ & $(n=255)$ \\
\hline Age & $75(54-89,7.3)$ & $73(56-88,7.3)$ & $73.1(66-86$, & $74(56-89,7.5)$ \\
Education & $15.7(4-20,3.0)$ & $14.6(4-20,3.3)$ & $14.7(4-20,3.2)$ & $15.5(4-20,3.1)$ \\
Female & $141(35.5 \%)$ & $79(41.6 \%)$ & $53(46.5 \%)$ & $101(39.6 \%)$ \\
Male & $256(64.5 \%)$ & $111(58.4 \%)$ & $61(53.5 \%)$ & $154(60.4 \%)$ \\
Caucasian & $371(93.5 \%)$ & $178(93.7 \%)$ & $106(93.0 \%)$ & $241(94.5 \%)$ \\
MMSE & $27.0(24-30$, & $26.6(24-30$, & $26.3(24-30$, & $27.0(24-30$, \\
& $1.8)$ & $1.8)$ & $1.8)$ & $1.8)$ \\
& & & & \\
& & & &
\end{tabular}

Note. For Age, Education, and MMSE, mean (range, standard deviation); for female, male, and Caucasian, number (percent). ADNI=Alzheimer's disease Neuroimaging Initiative; MMSE=Mini Mental Status Examination . 
Table 9. Neurocognitive Model using ADNI Backward Elimination Method

\begin{tabular}{|c|c|c|c|c|c|}
\hline Variable & $\begin{array}{l}\text { Mean } \\
\text { (Range) }\end{array}$ & $\beta$ & $p$ & $\begin{array}{l}\text { Example } \\
\text { Patient } \\
\text { Scores }\end{array}$ & $\begin{array}{l}\text { B X Example } \\
\text { Patient Score }\end{array}$ \\
\hline $\begin{array}{l}\text { Logical Memory } \\
\text { - II }\end{array}$ & $4.95(0-19)$ & -.177 & $<.001$ & 0 & 0 \\
\hline Clock Score & $4.20(0-5)$ & -.273 & .021 & 4 & -1.092 \\
\hline AVLT Total & $6.34(0-15)$ & -.205 & .012 & 5 & -1.025 \\
\hline Recall Trial 3 & & & & & \\
\hline $\begin{array}{l}\text { AVLT Total } \\
\text { Intrusion }\end{array}$ & $.46(0-9)$ & .203 & .072 & 0 & 0 \\
\hline Trial 5 & & & & & \\
\hline AVLT & $-.82(-2.67-$ & -.339 & .015 & -1.333 & .452 \\
\hline $\begin{array}{l}\text { Recognition } \\
\text { Z score }\end{array}$ & 1.33) & & & & \\
\hline AVLT & $2.08(0-15)$ & .112 & .027 & 0 & 0 \\
\hline Recognition & & & & & \\
\hline Errors & & & & & \\
\hline $\begin{array}{l}\text { Digit Span Z } \\
\text { Score }\end{array}$ & $.41(-2.67-2.67)$ & .219 & .080 & .333 & .073 \\
\hline $\begin{array}{l}\text { Animal Naming } \\
\text { Total }\end{array}$ & $15.82(2-60)$ & -.064 & .032 & 16 & -1.024 \\
\hline Animal Naming & $1.12(0-13)$ & .176 & .005 & 3 & .528 \\
\hline
\end{tabular}


Perseverations

Trails A

43.93 (15-296)

.014

.001

39

.546

BNT Total

2.65 (0-30)

$-.109$

$.003 \quad 4$

$-.436$

\section{Stimulus Cues}

\section{Constant}

$-.073$

$-.073$

Total

$-2.078$

Note. Mean $($ Range $)=$ mean and range. $\beta$ X Example Patient Score refers to multiplying unstandardized beta weight and patient score on associated variable. Total refers to sum of $\beta$ X Actual Score. $\beta=$ unstandardized beta weight, Actual Score=patient score on variable, $\mathrm{ADNI}=$ Alzheimer's disease Neuroimaging Initiative, AVLT=Rey Auditory Verbal Learning Test, BNT=Boston Naming Test. 
Table 10. Imaging Model using ADNI Backward Elimination Method

\begin{tabular}{llllll}
\hline Variable & Mean (Range) & $\boldsymbol{\beta}$ & $\boldsymbol{p}$ & Example & $\boldsymbol{\beta}$ X Example \\
& & & Patient & Patient Score \\
& & & & Score & \\
& & & & & \\
\hline Cumulative & $983(885-1036)$ & -.063 & $<.001$ & 991.279 & -62.45
\end{tabular}

Temporal Lobe

Atrophy

$\begin{array}{llllll}\text { Left } & 1799(816-2805) & -.002 & .001 & 1959.830 & -3.92\end{array}$

Hippocampus

Volume

$\begin{array}{llllll}\text { Right } & .282(.087-.488) & 19.218 & .003 & .374 & 7.18\end{array}$

Precentral Grey

Matter

$\begin{array}{llllll}\text { Right Frontal } & .432(.061-.743) & -19.653 & .001 & .528 & -10.38\end{array}$

Mid Orbital

Grey Matter

$\begin{array}{llllll}\text { Left Frontal } & .397(.114-.577) & 12.989 & .058 & .446 & 5.80\end{array}$

Inferior Orbital

Grey Matter

$\begin{array}{llllll}\text { Left Frontal } & .45(.13-.76) & 11.335 & .034 & .527 & 6.0\end{array}$

\section{Medial Orbital}

Grey Matter

$\begin{array}{llllll}\text { Right Posterior } & .333(.120-.557) & -15.869 & .003 & .366 & -5.80\end{array}$ 


\section{Cingulum}

Grey Matter

$\begin{array}{llllll}\text { Right Amygdala } & .62(.16-.86) & -7.958 & .024 & .741 & -5.90\end{array}$

Grey Matter

$\begin{array}{llllll}\text { Left Superior } \quad .30(.11-.55) & -6.119 & .103 & .387 & -2.37\end{array}$

Parietal Grey

Matter

$\begin{array}{llllll}\text { Left Superior } & .423(.151-.651) & -14.076 & .003 & .450 & -6.33\end{array}$

Temporal Grey

Matter

$\begin{array}{llllll}\text { Right } & .48(.18-.71) & 14.472 & .001 & .591 & 8.55\end{array}$

\section{Cerebellum}

Grey

Matter

Constant

68.101

68.101

Total

$-1.519$

Note. Mean $($ Range $)=$ mean and range for MCI patients. $\beta$ X Example Patient Score refers to multiplying unstandardized beta weight and patient score on associated variable, Total refers to sum of $\beta$ X Actual Score. Units of measurement for imaging variables are listed in $\mathrm{mm}^{3} . \beta=$ unstandardized beta weight, Actual Score=patient score on variable, ADNI=Alzheimer's disease Neuroimaging Initiative. 
Table 11. Final Model using ADNI Criteria, Hand Selected Method

\begin{tabular}{llllll}
\hline Variable & Mean (Range) & $\beta$ & $p$ & Example & $\beta$ X Example \\
& & Patient & Patient Score \\
& & Score & \\
& & &
\end{tabular}

AVLT Delayed $2.67(0-15)$

$-.392$

$.109 \quad 2$

$-.784$

Total

$\begin{array}{llllll}\text { AVLT Total } & .46(0-7) & 1.006 & .003 & 0 & 0\end{array}$

Intrusions

Trial 2

$\begin{array}{llllll}\text { Animal Naming } & 1.12(0-13) & .287 & .022 & 1 & .287\end{array}$

Perseverations

$\begin{array}{llllll}\text { BNT Total } & 2.65(0-30) & -.260 & .006 & 5 & -1.3\end{array}$

Semantic

Cues

$\begin{array}{llllll}\text { ADAS-Cog Total } & 18.7(0-30) & .115 & .030 & 24 & 2.76 \\ \text { Logical Memory } & 4.95(0-19) & -.052 & .639 & 7 & -.364 \\ \text { - II } & & & & & \\ \text { CDR Judgment } & .390(0-2) & 3.136 & .014 & .5 & 1.568 \\ \text { Cumulative } & 983(885-1036) & -.079 & .003 & 975.789 & -77.087\end{array}$

Temporal

Lobe Atrophy

$\begin{array}{llllll}\text { Right Precentral } & .282(.087-.488) & 32.456 & .001 & .342 & 11.1\end{array}$

Grey Matter 


\begin{tabular}{llllll}
\hline Front Mid & $.432(.061-.743)$ & -26.645 & .003 & .467 & -12.443
\end{tabular}

\section{Orbital Right}

\section{Grey Matter}

$\begin{array}{llllll}\text { Front Inferior } & .397(.114-.577) & 21.584 & .064 & .395 & 8.526\end{array}$

\section{Orbital Left}

\section{Grey Matter}

$\begin{array}{llllll}\text { Posterior Right } & .333(.120-.557) & -13.578 & .078 & .376 & -5.105\end{array}$

\section{Cingulum}

\section{Grey Matter}

$\begin{array}{llllll}\text { Left Superior } & .423(.151-.651) & -13.125 & .101 & .427 & -5.604\end{array}$

\section{Temporal}

\section{Grey Matter}

\section{Constant}

$76.564 \quad .004$

76.564

Total Equation

$-1.882$

Score

Note. See Statistical Analyses section for description of Hand Selected Method. Mean (Range $)=$ mean and range of MCI patients. $\beta$ X Example Patient Score refers to multiplying unstandardized beta weight and patient score on associated variable, Total refers to sum of $\beta$ X Actual Score. Units of measurement for imaging variables are listed in $\mathrm{mm}^{3} . \beta=$ unstandardized beta weight, Actual Score=patient score on variable, ADNI=Alzheimer's disease Neuroimaging Initiative, AVLT=Rey Auditory Verbal Learning Test, BNT=Boston Naming Test, ADAS-Cog=Alzheimer's disease Assessment Scale-Cognition, $\mathrm{CDR}=$ Clinical Dementia Rating. 
Table 12. Number and Percentage of Patients Identified as Normal, MCI, and Mild AD and Converting from MCI to dementia.

ADNI Criteria Proposed Proposed Proposed

Criteria \#1 Criteria \#2 Criteria \#3

\begin{tabular}{lllll}
\hline Normal & $229(27.9)$ & $229(27.9)$ & $230(28.0)$ & $230(28.0)$ \\
MCI & $397(48.4)$ & $191(23.3)$ & $115(14.0)$ & $255(31.1)$ \\
Mild AD & $192(23.4)$ & $201(24.5)$ & $183(22.3)$ & $191(23.3)$ \\
Unclassified & 0 & $197(24.1)$ & $290(35.5)$ & $142(17.4)$ \\
Conversions & $164(41.3)$ & $47(25.0)$ & $42(36.5)$ & $61(23.9)$
\end{tabular}

Note. For each cell, mean (\%). Conversions=patients converting from MCI to dementia. Proposed Criteria $\# 1=\leq-1$ SD on Logical Memory - II and $<6$ on the FAQ, Proposed Criteria $\# 2=\leq-1.5$ SD on Logical Memory - II and $<6$ on the FAQ, Proposed Criteria \#3 $=\leq-1.5 \mathrm{SD}$ in relation to VIQ on Logical Memory - II and $<6$ on the FAQ (See Table 7 for detailed description). $\mathrm{ADNI}=$ Alzheimer's disease neuroimaging initiative, $\mathrm{MCI}=$ mild cognitive impairment, $\mathrm{AD}=$ Alzheimer's disease, $\mathrm{FAQ}=$ Functional Activities Questionnaire, VIQ=verbal intelligence. 
Table 13. Final Model using ADNI Criteria, Backward Elimination Method

\begin{tabular}{llllll}
\hline Variable & Mean (Range) & $\beta$ & $p$ & Example & $\beta$ X Example \\
& & Patient & Patient Score \\
& & Score &
\end{tabular}

\begin{tabular}{llllll}
\hline CDR Judgment & $.390(0-2)$ & 2.544 & .019 & .5 &
\end{tabular}

$\begin{array}{llllll}\text { Logical Memory -II } & 4.95(0-19) & -.305 & .003 & 0 & 0\end{array}$

$\begin{array}{llllll}\text { Digit Span z score } & .414(-2.67-2.67) & .344 & .210 & 0 & 0\end{array}$

$\begin{array}{lllllll}\text { Animal Naming } & 15.82(2-60) & -.066 & .157 & 15 & -.99\end{array}$

Total Correct

$\begin{array}{llllll}\text { Animal Naming } & 1.12(0-13) & .246 & .034 & 2 & .492\end{array}$

Perseverations

$\begin{array}{llllll}\text { BNT Stimulus Cues } & 2.65(0-30) & -.265 & .002 & 7 & -1.855\end{array}$

$\begin{array}{llllll}\text { Cumulative } & 983(885-1036) & -.085 & .001 & 993.919 & -84.48\end{array}$

Temporal Lobe

Atrophy

Middle Left

$2.417(1.536-\quad-3.202 \quad .020 \quad 2.253 \quad-7.22$

Temporal Grey $\quad$ 2.951)

Matter

$\begin{array}{llllll}\text { Right Precentral } & .282(.087-.488) & 27.615 & .001 & .348 & 9.62\end{array}$

Grey Matter

$\begin{array}{llllll}\text { Front Mid Orbital } & .432(.061-.743) & -18.282 & .007 & .590 & -10.78\end{array}$

Right Grey Matter

$\begin{array}{llllll}\text { Total Intracranial } & 2.093(1.331- & 3.968 & .116 & 2.40 & 9.52\end{array}$ 
Volume

Constant

Total Equation

80.697

80.697

$-4.946$

Score

Note. Mean (Range)=mean and range of MCI patients. $\beta$ X Example Patient Score refers to multiplying unstandardized beta weight and patient score on associated variable, Total refers to sum of $\beta$ X Actual Score. Units of measurement for imaging variables are listed in $\mathrm{mm}^{3} . \beta=$ unstandardized beta weight, Actual Score=patient score on variable, ADNI=Alzheimer's disease Neuroimaging Initiative, BNT=Boston Naming Test, $\mathrm{CDR}=$ Clinical Dementia Rating. 
Table 14. Final Model using Proposed Criteria \#1(Model \#1).

\begin{tabular}{llllll}
\hline Variable & Mean (Range) & $\boldsymbol{\beta}$ & $\boldsymbol{p}$ & Example & $\boldsymbol{\beta}$ X Example \\
& & & & Patient & Patient Score \\
& & & \multicolumn{5}{c}{ Score } \\
& & & & & \\
\hline CDR Judgment & $.366(0-1)$ & 5.063 & .016 & 0 & 0 \\
FAQ & $1.85(0-5)$ & 1.172 & $<.001$ & 5 & 5.86 \\
Logical Memory I Total & $5.91(0-14)$ & -1.748 & $<.001$ & 8 & -13.984 \\
Logical Memory I z & $-1.43(-2.67-$ & 6.465 & .001 & -1.0 & -6.465 \\
score & $.67)$ & & & & \\
Clock Score & $4.15(1-5)$ & -.969 & .033 & 2 & -1.938 \\
Clock Copy Score & $4.69(2-5)$ & 5.783 & $<.001$ & 5 & 28.915 \\
AVLT Total Intrusion & $1.11(0-7)$ & .810 & .011 & 1 & .810 \\
Trial 6 & & & & & \\
Digit Span Total & $14.31(5-24)$ & 2.762 & $<.001$ & 10 & 27.62 \\
Digit Span Forward & $8.19(1-12)$ & -3.773 & .001 & 6 & -22.638 \\
Total & & & & & \\
Digit Span Forward & $6.50(3-8)$ & 1.603 & .067 & 5 & 8.015 \\
Length & & & & & \\
Digit Span Backward & $4.53(2-7)$ & -4.530 & .001 & 4 & -18.12 \\
Length & & & & & \\
Trails A & & & & & \\
\hline
\end{tabular}




\begin{tabular}{|c|c|c|c|c|c|}
\hline & $300)$ & & & & \\
\hline Trails A - B z score & $\begin{array}{l}-.65(-14.32- \\
1.85)\end{array}$ & -1.756 & .001 & 1.191 & -2.091 \\
\hline ADAS-Cog Total & $\begin{array}{l}20.29(3.33- \\
35.67)\end{array}$ & .619 & .001 & 20 & 12.38 \\
\hline $\begin{array}{l}\text { Right Precental Grey } \\
\text { Matter }\end{array}$ & $.25(.09-.38)$ & 121.155 & $<.001$ & .150 & 18.219 \\
\hline $\begin{array}{l}\text { Right Inferior Frontal } \\
\text { Gyrus Grey Matter }\end{array}$ & $.31(.11-.51)$ & -43.195 & .021 & .222 & -9.586 \\
\hline $\begin{array}{l}\text { Left Rolandic } \\
\text { Operculum Grey }\end{array}$ & $.35(.15-.61)$ & 83.692 & $<.001$ & .271 & 22.707 \\
\hline Matter & & & & & \\
\hline Right Mid Cingulum & $.43(.24-.62)$ & -70.822 & $<.001$ & .355 & -25.108 \\
\hline Grey Matter & & & & & \\
\hline Right Hippocampus & $.43(.19-.72)$ & -15.490 & .017 & .285 & -4.421 \\
\hline Grey Matter & & & & & \\
\hline Right Pallidum Grey & $.13(.01-.60)$ & 24.151 & $<.001$ & .428 & 10.342 \\
\hline Matter & & & & & \\
\hline Right Mid Temporal & $.43(.17-.67)$ & -64.065 & .006 & .348 & -22.314 \\
\hline Grey Matter & & & & & \\
\hline Left Mid Temporal & $.44(.19-.65)$ & 52.943 & .008 & .356 & 18.871 \\
\hline Grey Matter & & & & & \\
\hline Constant & & -29.464 & & & -29.464 \\
\hline
\end{tabular}


Note. Mean $($ Range $)=$ mean and range of MCI patients. $\beta$ X Example Patient Score refers to multiplying unstandardized beta weight and patient score on associated variable, Total refers to sum of $\beta$ X Actual Score. Units of measurement for imaging variables are listed in $\mathrm{mm}^{3} . \beta=$ unstandardized beta weight, Actual Score=patient score on variable, $\mathrm{CDR}=$ Clinical Dementia Rating, $\mathrm{AVLT}=$ Auditory Verbal Learning Test, ADAS$\mathrm{COG}=$ Alzheimer's disease Assessment Scale-Cognitive, $\mathrm{FAQ}=$ Functional Activities Questionnaire. 
Table 15. Final Model using Proposed Criteria \#2 (Model \#2).

\begin{tabular}{|c|c|c|c|c|c|}
\hline Variable & $\begin{array}{l}\text { Mean } \\
\text { (Range) }\end{array}$ & $\bar{\beta}$ & $p$ & $\begin{array}{l}\text { Example } \\
\text { Patient } \\
\text { Score }\end{array}$ & $\begin{array}{l}\beta \text { X Example } \\
\text { Patient Score }\end{array}$ \\
\hline$\overline{\text { FAQ }}$ & $2.18(0-5)$ & .873 & .008 & 3 & 2.619 \\
\hline Clock Copy Score & $4.70(3-5)$ & 1.737 & .130 & 5 & 8.585 \\
\hline AVLT Initial Recall z score & $\begin{array}{l}-.35(-2.67- \\
2.33)\end{array}$ & -2.015 & .002 & -1.667 & 3.358 \\
\hline $\begin{array}{l}\text { AVLT Total Errors Initial } \\
\text { Recall }\end{array}$ & $.52(0-5)$ & 1.164 & .021 & 1 & 1.164 \\
\hline $\begin{array}{l}\text { Animal Naming } \\
\text { Perseverations }\end{array}$ & $1.39(0-9)$ & .577 & .009 & 0 & 0 \\
\hline Trails A score & $\begin{array}{l}42.92(18- \\
150)\end{array}$ & .054 & .026 & 55 & 2.97 \\
\hline Trails A - B z score & $\begin{array}{l}-.85(-12.44- \\
1.8)\end{array}$ & .463 & .135 & -.277 & -.128 \\
\hline $\begin{array}{l}\text { BNT Phonemic Cue } \\
\text { Correct }\end{array}$ & $2.84(0-14)$ & -.412 & .023 & 1 & -.412 \\
\hline $\begin{array}{l}\text { Left Posterior Cingulum } \\
\text { Grey Matter }\end{array}$ & $.34(.14-.50)$ & -50.114 & .004 & .304 & -15.241 \\
\hline $\begin{array}{l}\text { Left Superior Occipital } \\
\text { Grey Matter }\end{array}$ & $.28(.10-.45)$ & -47.125 & .002 & .282 & -13.304 \\
\hline Left Supramarginal Gyrus & $.41(.22-.60)$ & 45.656 & .001 & .373 & 17.014 \\
\hline
\end{tabular}


Grey Matter

Right Central

$.25(.10-.46) \quad 37.009 \quad .004 \quad .210$

7.780

\section{Parahippocampal}

\section{Gyrus}

Constant

$-21.501$

$-21.501$

Total Equation Score

$-7.096$
Note. Mean (Range)=mean and range for MCI patients. $\beta$ X Example Patient Score refers to multiplying unstandardized beta weight and patient score on associated variable, Total refers to sum of $\beta$ X Actual Score. Units of measurement for imaging variables are listed in $\mathrm{mm}^{3} \cdot \beta=$ unstandardized beta weight, Actual Score $=$ patient score on variable, BNT=Boston Naming Test, FAQ=Functional Activities Questionnaire, AVLT=Auditory Verbal Learning Test. 
Table 16. Final Model using Proposed Criteria \#3 (Model \#3).

\begin{tabular}{|c|c|c|c|c|c|}
\hline Variable & Mean (Range) & $\beta$ & $p$ & $\begin{array}{l}\text { Example } \\
\text { Patient } \\
\text { Score }\end{array}$ & $\begin{array}{l}\beta \text { X Example } \\
\text { Patient Score }\end{array}$ \\
\hline $\begin{array}{l}\text { FAQ } \\
\end{array}$ & $1.85(0-5)$ & .371 & .002 & 3 & 1.113 \\
\hline ADAS-Cog Total & $18.73(3-35.6)$ & .125 & .001 & 27 & 3.375 \\
\hline Right & $3311(1649-4835)$ & -.001 & .003 & 2676 & -2.676 \\
\hline $\begin{array}{l}\text { Hippocampal } \\
\text { Volume }\end{array}$ & & & & & \\
\hline $\begin{array}{l}\text { Right Mid } \\
\text { Frontal Grey } \\
\text { Matter }\end{array}$ & $.35(.07-.61)$ & -10.02 & .007 & .332 & -3.329 \\
\hline $\begin{array}{l}\text { Left Heschl's } \\
\text { Gyrus Grey }\end{array}$ & \multicolumn{4}{|c|}{ Gyrus Grey } & 4.898 \\
\hline \multicolumn{6}{|l|}{ Matter } \\
\hline $\begin{array}{l}\text { Right Inferior } \\
\text { Parietal Grey }\end{array}$ & $.36(.15-.60)$ & 8.235 & .098 & .317 & 2.614 \\
\hline \multicolumn{6}{|l|}{ Matter } \\
\hline Constant & & -8.562 & & & -8.562 \\
\hline Total Equation & & & & & -2.567 \\
\hline Score & & & & & \\
\hline
\end{tabular}

Note. Mean (Range)=mean and range of MCI patients. $\beta$ X Example Patient Score refers to multiplying unstandardized beta weight and patient score on associated variable, Total 
refers to sum of $\beta$ X Actual Score. Units of measurement for imaging variables are listed in $\mathrm{mm}^{3} . \beta=$ unstandardized beta weight, Actual Score=patient score on variable, $\mathrm{FAQ}=$ Functional Activities Questionnaire, ADAS-COG=Alzheimer's disease Assessment Scale-Cognitive. 
Table 17. Performance of Models across Classification System ( $\beta$, $p$ ).

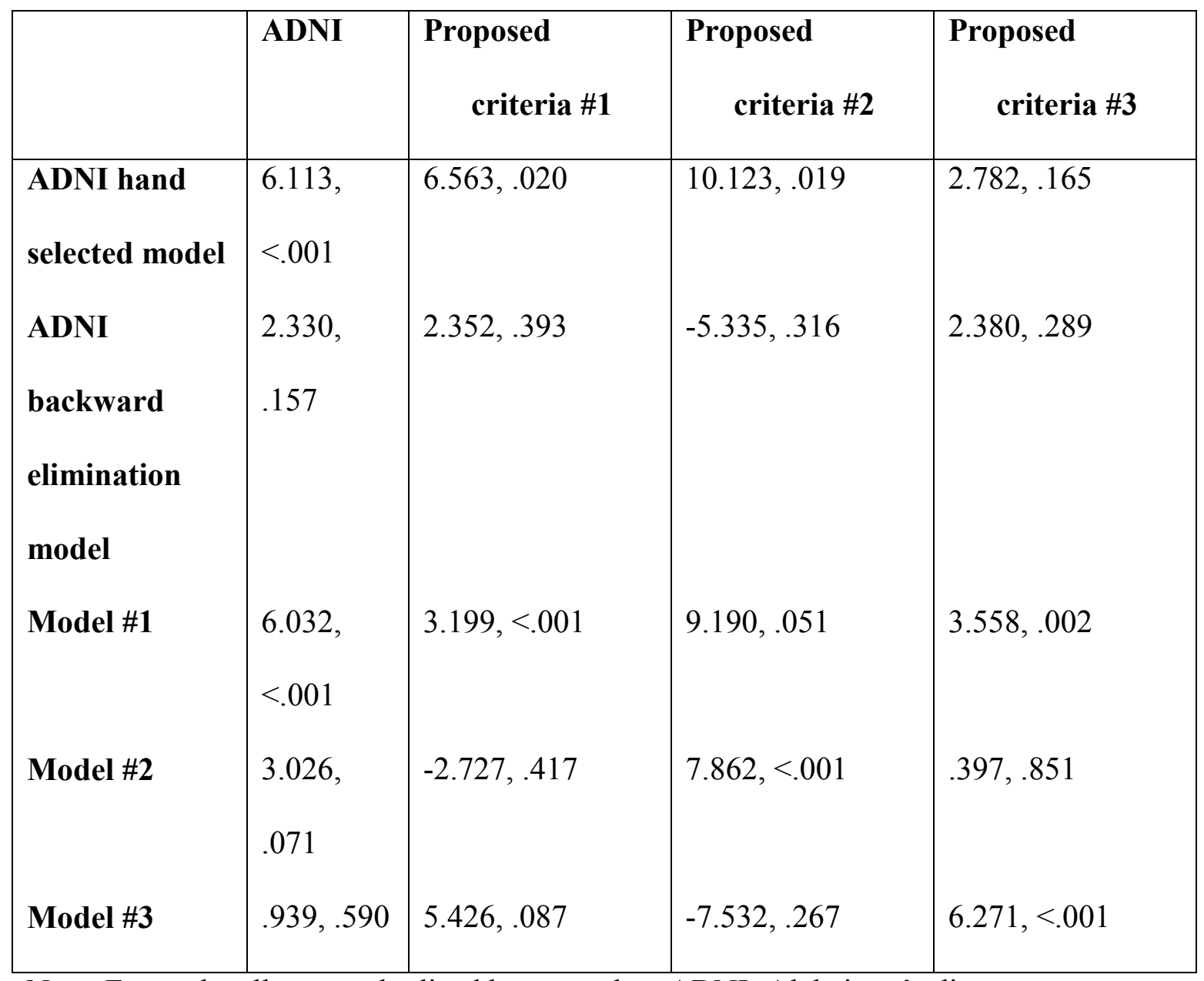

Note. For each cell, unstandardized beta, $p$ value. ADNI=Alzheimer's disease

Neuroimaging Initiative, $\beta=$ unstandardized beta. 
Table 18. Performance of Models across Classification Systems (Pseudo R2 ${ }^{2}$ AUC).

\begin{tabular}{lllll}
\hline & ADNI & $\begin{array}{l}\text { Proposed } \\
\text { criteria } \# \mathbf{1}\end{array}$ & $\begin{array}{l}\text { Proposed criteria } \\
\mathbf{\# 2}\end{array}$ & $\begin{array}{l}\text { Proposed criteria } \\
\text { ( }\end{array}$ \\
& & & & \\
\hline ADNI hand & $.61, .93$ & $.40, .86$ & $.51, .89$ & $.35, .81$ \\
selected model & & & & \\
ADNI & $.50, .91$ & $.31, .82$ & $.33, .81$ & $.26, .76$ \\
backward & & & & \\
elimination & & & & \\
model & & & & \\
Model \#1 & $.39, .88$ & $.74, .97$ & $.62, .94$ & $.47, .89$ \\
Model \#2 & $.22, .81$ & $.39, .87$ & $.77, .97$ & $.25, .80$ \\
Model \#3 & $.23, .82$ & $.35, .86$ & $.31, .83$ & $.41, .88$ \\
\hline
\end{tabular}

Note. For each cell, pseudo $R^{2}$, AUC. Yellow highlighting indicates the pseudo- $\mathrm{R}^{2}$ and AUC of each model in relation to the Proposed Criteria from which they were derived. Blue highlighting indicates the best and red highlighting indicates the worst pseudo- $\mathrm{R}^{2}$ and AUC of a model in relation to a validation sample/Proposed Criteria from which it was not derived. $\mathrm{ADNI}=\mathrm{Alzheimer}$ 's disease Neuroimaging Initiative, $\mathrm{AUC}=$ area under the curve. 
Table 19. User-Friendly Model

\begin{tabular}{llll}
\hline Variable & Mean (Range) & $\boldsymbol{\beta}$ & $\boldsymbol{p}$ \\
\hline FAQ & $4.29(0-28)$ & .083 & $<.001$ \\
Logical Memory - II & $4.95(0-19)$ & -.158 & .002 \\
Clock Score & $4.20(0-5)$ & -.176 & .133 \\
AVLT Delay Total & $2.67(0-15)$ & -.167 & .035 \\
Trails A & $43.93(15-296)$ & .010 & .037 \\
ADAS-Cog Total & $18.72(0-67)$ & .034 & .129 \\
Constant & & -3.456 & \\
\end{tabular}

Note. Mean $($ Range $)=$ mean and range of MCI patients. $\beta=$ unstandardized beta weight, FAQ $=$ Functional Activities Questionnaire, ADAS-Cog=Alzheimer's disease Assessment Scale-Cognitive. 


\section{REFERENCES}

Aggarwal, N. T., Wilson, R. S., Beck, T. L., Bienias, J. L., \& Bennett, D. A. (2005). Mild cognitive impairment in different functional domains and incident Alzheimer's disease. Journal of Neurology, Neurosurgery, and Psychiatry, 76, 1479-1484.

Albert, M. S., DeKosky, S. T., Dickson, D., Dubois, B., Feldman, H. H., Fox, N. C.,...Phelps, C. H. (2011). The diagnosis of mild cognitive impairment due to

Alzheimer's disease: Recommendations from the National Institute on Aging and Alzheimer's Association workgroup. Alzheimer's \& Dementia, 7, 270-279.

Altman, D. G. \& Bland, J. M. (1994). Statistics Notes: Diagnostic tests 3: receiver operating characteristics plots. British Medical Journal, 188, 309.

American Psychiatric Association. (2000). Diagnostic and statistical manual of mental disorders ( $4^{\text {th }}$ ed., text rev.). Washington, DC: Author.

American Psychological Association, Presidential Task Force on the Assessment of AgeConsistent Memory Decline and Dementia (2011). Guidelines for the evaluation of dementia and age-related cognitive decline. Washington, DC: American Psychological Association.

Andersen, K., Launer, L. J., Dewey, M. E., Letenneur, L., Ott, A., Copeland, J. R.,...Hofman A. (1999). Gender differences in the incidence of AD and vascular dementia: The EURODEM Studies. EURODEM Incidence Research Group. Neurology, 53, 1992-1997. 
Anoop, A., Singh, P. K., Jacob, R. S., \& Maji, S. K. (2010). CSF Biomarkers for Alzheimer's Disease Diagnosis. International Journal of Alzheimer's Disease, doi:10.4061/2010/606802.

Applegate, W. B., Pressel, S., Wittes, J., Luhr, J., Shekelle, R. B., Camel, G. H.,...Perry, H. M. (1994). Impact of the treatment of isolated systolic hypertension on behavioral variables. Results from the systolic hypertension in the elderly program. Archives of Internal Medicine, 154, 2154-2160

Arai, H., Clark, C. M., Ewbank, D. C., Takase, S., Higuchi, S., \& Miura, M. (1998). Cerebrospinal Fluid Tau Protein as a Potential Diagnostic Marker in Alzheimer's Disease. Neurobiology of Aging, 19(2), 125-126.

Arai, H., Morikawa, Y., Higuchi, M., Matsui, T., Clark, C. M., \& Miura, M. (1997). Cerebrospinal fluid tau levels in neurodegenerative diseases with distinct taurelated pathology. Biochemical Biophysical Resource Community, 236(2), 262-264.

Arai, H., Terajima, M., Miura, M., Higuchi, S., Muramatsu, T., \& Machida, N. (1995). Tau in cerebrospinal fluid: a potential diagnostic marker in Alzheimer's disease. Annals of Neurology, 38(4), 649-652.

Arai, H., Terajima, M., Miura, M., Higuchi, S., Muramatsu, T., \& Matsushita, S. (1997). Effect of genetic risk factors and disease progression on the cerebrospinal fluid tau levels in Alzheimer's disease. Journal of the American Geriatric Society, 45(10), 1228-1231.

Artero, S., Petersen, R., Touchon, J., \& Ritchi, K. (2006). Revised criteria for mild 
cognitive impairment: validation within a longitudinal population study.

Dementia and Geriatric Cognitive Disorders, 22, 465-470.

Azad, N. A., Al Bugami, M., \& Loy-English. (2007). Gender Differences in Dementia Risk Factors. Gender Medicine, 4, 120-129.

Bachman, D. L., Wolf, P. A., Linn, R. T., Knoefel, J. E., Cobb, J. L., Belanger, A. J.,... D’Agostino, R. B. (1993). Incidence of dementia and probable Alzheimer's disease in a general population: the Framingham Study. Neurology, 43, 515-519.

Barnes, D. E., Alexopoulos, G. S., Lopez, O. L., Williamson, J. D., \& Yaffe, K. (2006). Depressive symptoms, vascular disease, and mild cognitive impairment: findings from the Cardiovascular Health Study. Archives of General Psychiatry, 63, 273-279.

Barnes, D. E., Covinsky, K. E., Whitmer, R. A., Kuller, L. H., Lopez, O. L., \& Yaffe, K. (2009). Predicting risk of dementia in older adults: the late-life dementia risk index. Neurology 73, 173-179.

Becker, J. T., Chang, Y. F., Lopez, O. L.,, Dew, M. A., Sweet, R. A., Barnes, D.,...Reynolds, C. F. (2009). Depressed mood is not a risk factor for incident dementia in a community-based cohort. American Journal of Geriatric Psychiatry, 17, 653-663.

Berg, L. (1988). Clinical Dementia Rating (CDR). Psychopharmacological Bulletin, 24(4), 637-639.

Bernick, C., Katz, R., Smith, N. L., Rapp, S., Bhadelia, R., Carlson, M., \& Kuller, L., for the Cardiovascular Health Study Collaborative Research Group. (2005). Statin and cognitive function in the elderly: The Cardiovascular Health Study. 
Neurology, 65, 1388-1394.

Blacker, D., Albert, M. S., Bassett, S. S., Go, R. C., Harrell, L. E., \& Folstein, M. F. (1994). Reliability and validity of NINCDS-ADRDA criteria for Alzheimer's disease. The National Institute of Mental Health Genetics Initiative. Archives of Neurology, 51, 1198-1204.

Blacker, D., Lee, H., Muzikansky, A., Martin, E. C., Tanzi, R., McArdle, J. J.,...Albert, M. (2007). Neuropsychological measures in normal individuals that predict subsequent cognitive decline. Archives of Neurology, 64, 862-871.

Blennow, K., Hampel, H., Weiner, M., \& Zetterberg, H. (2010). Cerebrospinal fluid and plasma biomarkers in Alzheimer disease. Nature Reviews Neurology, 6, 131-144.

Brayne, C., Gill, C., Huppert, F. A., Barkley, C., Gehlhaar, E., Girling, D. M.,...Paykel, E. S. (1995). Incidence of clinically diagnosed subtypes of dementia in an elderly population. Cambridge Project for Later Life. British Journal of Psychiatry, 167, $255-262$.

Brayne, C., Ince, P. G., Keage, H. A. D., McKeith, I. G., Matthews, F. E., Polvikoski, T., \& Sulkava, R., for the EClipSE Collaborative Members. (2010). Education the brain and dementia: neuroprotection or compensation? Brain, 8, 2210-2216.

Brodaty, H., Fay, L. L., Gibson, L., \& Burns, K. (2006). What is the best dementia screening instrument for general practitioners to use? American Journal of Geriatric Psychiatry 14, 391-400.

Busse, A., Bischkopf, J., Riedel-Heller, S. G., \& Angermeyer, M. C. (2003). Mild cognitive impairment: prevalence and incidence according to different diagnostic 
criteria - results of the Leipzig Longitudinal Study of the Aged (LEILA75+). British Journal of Psychiatry, 182, 449-454.

Busse, A., Hensel, A., Guhne, U., Angermeyer, M. C., \& Riedel-Heller, S. G. (2006). Mild cognitive impairment: long-term course of four clinical subtypes. Neurology, 67, 2176- 2185.

Butters, N., Granholm, E., Salmon, D. P., Grant, I., \& Wolfe, J. (1987). Episodic and semantic memory: a comparison of amnesic and demented patients. Journal of Clinical and Experimental Neuropsychology, 9(5), 479-497.

Carlson, M. C., Xue, Q., Zhou, J., \& Fried, L. P. (2009). Executive Decline and Dysfunction Precedes Declines in Memory: The Women's Health and Aging Study II. The Journals of Gerontology Series A: Biological Sciences and Medical Sciences, 64, 110-117.

Caracciolo, B., Palmer, K., Monastero, R., Winblad, B., Backman, L., \& Fratiglioni, L. (2008). Occurrence of cognitive impairment and dementia in the community: a 9year-long prospective study. Neurology, 70, 1778-1785.

Castano, E. M., Prelli, F., Soto, C., Beavis, R., Matsubara, E., Shoji, M., \& Franqione, B. (1996). The length of amyloid-beta in hereditary cerebral hemorrhage with amyloidosis, Dutch type. Implications for the role of amyloid-beta 1-42 in Alzheimer's disease. Journal of Biological Chemistry, 271, 32185-32191.

Chaves, M. L., Camozzato, A. L., Godinho, C., Piazenski, I., \& Kaye, J. (2009). Incidence of mild cognitive impairment and Alzheimer disease in southern Brazil. Journal of Geriatric Psychiatry and Neurology, 22, 181-187.

Clark, C. M., Ewbank, D., Peskind, E., Galasko, D., Morris, J. C., \& Farlow, M. (2003). 
CSF tau and Amyloid- $\beta$ : How well do these biomarkers reflect autopsy confirmed dementia diagnosis. Archives of Neurology, 60, 1696-1702.

Clark, C. M., Sheppard, L., Fillenbaum, G. G., Galasko, D., Morris, J. C., Koss, E.,...Heyman, A. (1999). Variability in annual Mini-Mental State Examination score in patients with probable Alzheimer disease: a clinical perspective of data from the Consortium to Establish a Registry for Alzheimer's Disease. Archives of Neurology, 56, 857-862.

Clarke, D., Morgan, K., Lilley, J., Arie, T., Jones, R., Waite, J., \& Prettyman, R. (1996). Dementia and 'borderline dementia' in Britain: 8-year incidence and post-screening outcomes. Psychological Medicine, 26, 829-835.

Cockburn, J., Keene, J., Hope, T., \& Smith, P. (2000). Progressive decline in NART score with increasing dementia severity. Journal of Clinical and Experimental Neuropsychology, 22, 508-517.

Coker, L. H. \& Shumaker, S. A. (2003). Type 2 diabetes mellitus and cognition: An understudied issue in women's health. Journal of Psychosomatic Research, 54, 129-139.

Crawford, J. R., Millar, J., \& Milne, A. B. (2001). Estimating premorbid IQ from demographic variables: A comparison of a regression equation vs. clinical judgment. British Journal of Clinical Psychology, 40, 97-105.Dawes, R. M. (1994). House of cards: Psychology and psychotherapy built on myth. New York, NY: Free Press.

Crum, R. M., Anthony, J. C., Bassett, S. S., \& Folstein, M. F. (1993) Population-based 
norms for the mini-mental state examination by age and educational level. Journal of the American Medical Association, 18, 2386-2391.

Dawes, R.M. (2005). The ethical implications of Paul Meehl's work on comparing clinical versus statistical prediction methods. Journal of Clinical Psychology, $61,1245-1255$.

Dawes, R.M., Faust. D. \& Meehl, P.E. (1989). Clinical versus statistical judgment. Science, 31, 1668-1674.

Del Ser, T., Hachinski, V., Merskey, H., \& Munoz, D. G. (1999). An autopsy-verified study of the effect of education on degenerative dementia. Brain, 122, 2309-2319.

Devanand, D. P., Pradhaban, G., Liu, X., Khandji, A., De Santi, S., Segal, S.,...de Leon, M. J. (2007). Hippocampal and entorhinal atrophy in mild cognitive impairment: prediction of Alzheimer's disease. Neurology, 68, 828-836.

Devanand, D. P., Liu, X., Tabert, M. H., Pradhaban, G., Cuasay, K., Bell, K.,...Pelton, G. H. (2008). Combining early markers strongly predicts conversion from mild cognitive impairment to Alzheimer's disease. Biological Psychiatry, 64, 871-879.

Devore, E., Ridker, P., \& Grodstein, F. (2004). Plasma cholesterol levels and cognitive function in aging women. In: Program and abstracts of the $9^{\text {th }}$ International Conference on Alzheimer's Disease and Related Disorders; Philadelphia, Pa. Abstract O3-01-02.

Dickerson, B. C. \& Wolk, D. A. (2013). Biomarker-based prediction of progression in MCI: Comparison of AD signature and hippocampal volume with spinal fluid amyloid- $\beta$ and tau. Frontiers in Aging Neuroscience, doi:10.3389/fnagi.2013.00055. 
Drzezga, A., Lautenschlager, N., Siebner, H., Riemenschneider, M., Willoch, F., Minoshima, S.,...Kurz, A. (2003). Cerebral metabolic changes accompanying conversion of mild cognitive impairment into Alzheimer's disease: a PET followup study. European Journal of Nuclear Medicine and Molecular Imaging, 30, 1104-1113.

Farrer, L. A., Cupples, L. A., Haines, J. L., Hyman, B., Kukull, W. A., Mayeux, R.,...van Duijn, C. M., for the APOE and Alzheimer Disease Meta Analysis Consortium. (1997). Effects of age, sex, and ethnicity on the association between apolipoprotein E genotype and Alzheimer disease. A meta-analysis. Journal of the American Medical Association, 278, 1349-1356.

Fassbender, K., Mielke, O., Bertsch, T., Nafe, B., Fröschen, S., \& Hennerici, M. (1999). Homocysteine in cerebral macroangiography and microangiopathy. Lancet, 353, 1586-1587.

Fleisher, A., Grundman, M., Jack, C. R. Jr, Petersen, R. C., Taylor, C., Kim, H. T.,...Thal, L. J., for the Alzheimer's Disease Cooperative Study. (2005). Sex, apolipoprotein E epsilon 4 status, and hippocampal volume in mild cognitive impairment. Archives of Neurology, 62, 953-957.

Fleisher, A. S., Sowell, B. B., Taylor, C., Gamst, A. C., Petersen, R. C., \& Thal, L. J., for the Alzheimer's Disease Cooperative Study. (2007). Clinical predictors of progression to Alzheimer disease in amnestic mild cognitive impairment. Neurology, 2007, 68, 1588-1595.

Flicker, C., Ferris, S. H., \& Reisberg, B. (1991). Mild cognitive impairment in the elderly: predictors of dementia. Neurology, 41, 1006-1009. 
Folstein, M. F., Folstein, S. E., \& McHugh, P. R. (1975). Mini Mental State: A practical method for grading the cognitive state of patients for the clinician. Journals of Gerontology: Series B: Psychological Sciences \& Social Sciences, 12, 189-198.

Forette, F., Seux, M. L., Staessen, J. A., Thijs, L., Babarskiene, M. R., Babeanu, S.,...Birkenhager, W. H., for the Systolic Hypertension in Europe Investigators. The prevention of dementia with antihypertensive treatment: New evidence from the Systolic Hypertension in Europe (Syst-Eur) study. Archives of Internal Medicine, 162, 2046-2052.

Frank, R. A., Galasko, D., Hampel, H., Hardy, J., de Leon, M. J., \& Mehta, P. D. (2003). Biological markers for therapeutic trials in Alzheimer's disease. Proceedings of the biological markers working group; NIA initiative on neuroimaging in Alzheimer's disease. Neurobiology of Aging, 24, 521-536.

Fratiglioni, L., Viitanen, M., von Strauss, E., Tontodonati, V., Herlitz, A., \& Winblad, B. (1997). Very old women at highest risk of dementia and Alzheimer's disease: incidence data from the Kungsholmen project, Stockholm. Neurology, 48, 132138.

Ganguli, M., Dodge, H. H., Chen, P., Belle, S., \& DeKosky, S. T. (2000). Ten-year incidence of dementia in a rural elderly US community population: the MoVIES project. Neurology, 54, 1109-1116.

Ganguli, M., Dodge, H. H., Shen, C., \& DeKosky, S. T. (2004). Mild cognitive impairment, amnestic type: an epidemiologic study. Neurology, 63, 115-121.

Geda, Y. E., Knopman, D. S., Mrazek, D. A., Jicha, G. A., Smith, G. E., Negash, 
S.,...Rocca, W. A. (2006). Depression, apolipoprotein E genotype, and the incidence of mild cognitive impairment: a prospective cohort study. Archives of Neurology, 63, 435-440.

Goldberg, T. E., Koppel, J., Keehlisen, L., Christen, E., Dreses-Werringloer, U., Conejero-Goldberg, C.,...Davies, P. (2010). Performance-Based Measures of Everyday Function in Mild Cognitive Impairment. American Journal of Psychiatry, 167, 845-853.

Goldberg TE, Ragland JD, Torrey EF, Gold JM, Bigelow LB, Weinberger DR. (1990). Neuropsychological assessment of monozygotic twins discordant for schizophrenia. Archives of General Psychiatry, 47, 1066-1072.

Gomar, J. J., Bobes-Bascaran, M. T., Conejero-Goldberg, C., Davies, P., \& Goldberg, T. E. (2011). Utility of Combinations of Biomarkers, Cognitive Markers, and Risk Factors to Predict Conversion From Mild Cognitive Impairment to Alzheimer Disease in Patients in the Alzheimer's Disease Neuroimaging Initiative. Archives of General Psychiatry, 68, 961-969.

Goodglass, H., \& Kaplan, E. (1983). The assessment of aphasia and related disorders. Philadelphia: Lea \& Febiger.

Gregg, E. W., Yaffe, K., Cauley, J. A., Rolka, D. B., Blackwell, T. L., Narayan, K. M.., \& Cummings, S. R., for the Study of Osteoporotic Fractures Research Group. (2000). Is diabetes associated with cognitive impairment and cognitive decline among older women? Archives of Internal Medicine, 160, 174-180.

Griffin, S. L., Mindt, M. R., Rankin, E. J., Ritchie, A. J., \& Scott, J. G. (2002). 
Estimating premorbid intelligence: Comparison of traditional and contemporary methods across the intelligence continuum. Archives of Clinical Neuropsychology, 17, 497-507.

Hagnell, O., Ojesjo, L., \& Rorsman, B. (1992). Incidence of dementia in the Lundby study. Neuroepidemiology, 11, 61-66.

Heaton, R. K., Velin, R. A., McCutchan, J. A., Gulevich, S. J., Atkinson, J. H., \& Wallace, M. R.,...Grant I. Neuropsychological impairment in human immunodeficiency virus infection: implications for employment. Psychosomatic Medicine, 56, 8-17.

Hebert, L. E., Scherr, P. A., Bienias, J. L., Bennett, D. A., \& Evans, D. A. (2003). Alzheimer disease in the US population: prevalence estimates using the 2000 census. Archives of Neurology, 60, 1119-1122.

Henderson, A. S., Korten, A. E., Jacomb, P. A., Mackinnon A. J., Jorm, A. F., Christensen, H., \& Rodgers, B. (1997). The course of depression in the elderly: a longitudinal community-based study in Australia. Psychological Medicine, 27, 119-129.

Isbir, T., Agachan, B., Yilmaz, H., Aydin, M., Kara, I., Eker, E., \& Eker, D. (2001). Apolipoprotein-E gene polymorphism and lipid profiles in Alzheimer's disease. American Journal Alzheimer's Disease and Other Dementia, 16, 77-81.

Jack, C. R. Jr., Knopman, D. S., Jagust, W. J., Shaw, L. M., Aisen, P. S., Weiner, M. W.,...Trojanowski, J. Q. (2010). Hypothetical model of dynamic biomarkers of the Alzheimer's pathological cascade. Lancet Neurology, 9, 119-128.

Jack, C. R. Jr., Petersen, R. C., Xu, Y. C., O’Brien, P. C., Smith, G. E., Ivnik, R. 
J.,...Kokmen, E. (1999). Prediction of AD with MRI-based hippocampal volume in mild cognitive impairment. Neurology, 52, 1397-1403.

Jorm, A. F. (2001). History of depression as a risk factor for dementia: an updated review. Australian and New Zealand Journal of Psychiatry, 35, 776-781.

Kahokehr, A., Siegert, R. J., \& Weatherall, M. (2004). The frequency of executive cognitive impairment in elderly rehabilitation inpatients. Journal of Geriatric Psychiatry and Neurology 17(2), 68-72.

Kaplan, E., Goodglass, H., \& Weintraub S. (1983). The Boston Naming Test. Philadelphia, PA: Lea \& Febiger.

Kaufer, D. I., Cummings, J. L., Ketchel, P., Smith, V., MacMillan, A., \& Shelley, T. (2000). Validation of the NPI-Q, a brief clinical form of the Neuropsychiatric Inventory. Journal of Neuropsychiatry and Clinical Neuroscience, 12, 233-239.

Kearney, P. M., Whelton, M., Reynolds, K., Mutner, P., Whelton, P. K., \& He, J. (2005). Global burden of hypertension: Analysis of worldwide data. Lancet, 365, 217223.

Kiely, K. M., Luszcz, M. A., Piguet, O., Christensen, H., Bennett, H., \& Anstey, K. J. (2011). Functional equivalence of the National Adult Reading Test (NART) and Schonell reading tests and NART norms in the Dynamic Analyses to Optimise Ageing (DYNOPTA) project. Journal of Clinical and Experimental Neuropsychology, 33, 410-421.

Kivipelto, M., Helkala, E. L., Laakso, M. P, Hanninen, T., Hallikainen, M., Alhainen, K.,...Nissinen, A. (2001). Midlife vascular risk factors and Alzheimer's disease in 
later life: Longitudinal, population based study. British Medical Journal, 322, $1447-1451$.

Larrieu, S., Letenneur, L., Orgogozo, J. M., Fabrigoule, C., Amieva, H., Le Carret, N.,...Dartigues, J. F. (2002). Incidence and outcome of mild cognitive impairment in a population-based prospective cohort. Neurology, 59, 1594-1595.

Letenneur, L., Commenges, D., Dartigues, J. F., \& Barberger-Gateau, P. (1994). Incidence of dementia and Alzheimer's disease in elderly community residents of southwestern France. International Journal of Epidemiology, 23, 1256-1261.

Letenneur, L., Gilleron, V., Commenges, D., Helmer, C., Orgogozo, J. M., \& Dartigues, J. F. (1999). Are sex and education level independent predictors of dementia and Alzheimer's disease? Incidence data from the PAQUID project. Journal of Neurology, Neurosurgery, and Psychiatry, 66, 177-183.

Liao, D., Cooper, L., Cai, J., Toole, J., Bryan, N., Burke, G.,...Heiss, G. (1997). The prevalence and severity of white matter lesions, their relationship with age, ethnicity, gender, and cardiovascular disease risk factors: The ARIC Study. Neuroepidemiology, 16, 149-162.

Lobo, A., Launer, L. J., Frattiglioni, L., Anderson, K., Di Carlo, A. Breteler, M. M.,...Hofman, A., for the Neurologic Diseases in the Elderly Research Group. (2000). Prevalence of dementia and major subtypes in Europe: A collaborative study of population-based cohorts. Neurology, 54, 4-9.

Loewenstein, D. A., Argüelles, S., Bravo, M., Freeman, R. Q., Argüelles, T., Acevedo, A., \& Eisdorfer, C. (2001). Caregivers' judgments of the functional abilities of the Alzheimer's disease patient: a comparison of proxy reports and objective 
measures. Journal of Gerontology Series B: Psychological Science and Social Science, 56, 78-84.

Longstreth, W. T. Jr., Manolio, T. A., Arnold, A., Burke, G. L., Bryan, N., \& Jungreis, C. A. (1996). Clinical correlates of white matter findings on cranial magnetic resonance imaging of 3301 elderly people. The Cardiovascular Health Study. Stroke, 27, 1274-1282.

Lopez, O. L., Jagust, W. J., DeKosky, S. T., Becker, J. T., Fitzpatrick, A., Dulberg, C.,...Kuller, L. H. (2003). Prevalence and Classification of Mild Cognitive Impairment in the Cardiovascular Health Study Cognition Study: Part 1. Archives of Neurology, 60, 1385-1389.

Lorentz, W. J., Scanlan, J. M., \& Borson, S. (2002). Brief screening tests for dementia. Canadian Journal of Psychiatry 47, 723-733.

Luck, T., Riedel-Heller, S. G., Kaduszkiewicz, H., Bickel, H., Jessen, F., Pentzek, M.,...Weyerer, S., AgeCoDe Group. (2007). Mild cognitive impairment in general practice: age-specific prevalence and correlate results from the German study on ageing, cognition and dementia in primary care patients (Age-CoDe). Dementia and Geriatric Cognitive Disorders, 24, 307-316.

Luck, T., Luppa, M., Briel, S., \& Riedel-Heller, S. G. (2010). Incidence of mild cognitive impairment: A systematic review. Dementia and Geriatric Cognitive Disorders, 29, 164-175.

Manly, J. J., Tang, M. X., Schupf, N., Stern, Y., Vonsattel, J. P. G., \& Mayeux, R. (2008). Frequency and course of mild cognitive impairment in a multiethnic community. Annals of Neurology, 63, 494-506. 
Mattsson, N., Zetterberg, H., Hansson, O., Andreasen, N., Parnetti, L., Jonsson, M.,...Blennow, K. (2009). CSF biomarkers and incipient Alzheimer disease in patients with mild cognitive impairment. Journal of the American Medical Association, 302, 385-393.

McGurn, B., Starr, J. M., Topfer, J. A., Partie, A., Whiteman, M. C., \& Lemmon, H. A. (2004). Pronunciation of irregular words is preserved in dementia, validating premorbid IQ estimation. Neurology, 62, 1184-1186.

McKhann, G., Drachman, D., Folstein, M., Katzman, R., Price, D., \& Stadlan, E. M. (1984). Clinical diagnosis of Alzheimer's disease: report of the NINCDS-ADRA Work Group under the auspices of Department of Health and Human Services Task Force on Alzheimer's Disease. Neurology, 34, 939-944.

Meehl, P. E. (1954/1996). Clinical versus statistical prediction: A theoretical analysis and review of the evidence. Northvale, NJ: Jason Aronson.

Mielke, M. M., Zandi, P. P., Sjogren, M., Gustafson, D., Ostling, S., Steen, B., \& Sloog, I. (2005). High total cholesterol levels in late life associated with a reduced risk of dementia. Neurology, 64, 1689-1695.

Modrego, P. J. \& Ferrandez, J. (2004). Depression in patients with mild cognitive impairment increases the risk of developing dementia of Alzheimer type: a prospective cohort study. Archives of Neurology, 61, 1290-1293.

Morgan, K., Lilley, J. M., Arie, T., Byrne, E. J., Jones, R., \& Waite, J. (1993). Incidence of dementia in a representative British sample. British Journal of Psychiatry, 163, $467-470$.

Morin-Martin, M., Gonzalez-Santiago, R., Gil-Nunez., A. C., \& Vivancos-Mora J. 
(2003). Women and strokes. Hospital epidemiology in Spain. Revue Neurologique, 37, 701-705.

Moroney, J. T., Tang, M. X., Berglund, L., Small, S., Merchant, C., Bell, K.,...Mayeux, R. (1999). Low density lipoprotein cholesterol and the risk of dementia with stroke. Journal of the American Medical Association, 282, 254-260.

Morris, J. C. (1997). Clinical dementia rating: a reliable and valid diagnostic and staging measure of dementia of the Alzheimer type. International Psychogeriatrics, 9, 173-176.

Nelson, H. E., \& O'Connell, A. (1978). Dementia: the estimation of premorbid intelligence levels using the New Adult Reading Test. Cortex, 14, 234-244.

O’Carroll, R. E., Prentice, N., Murray, C., Van Beck, M., Ebmeier, K. P., \& Goodwin, G. M. (1995). Further evidence that reading ability is not preserved in Alzheimer's disease. British Journal of Psychiatry, 167, 659-662.

Okereke, O., Hankinson, S. E., Hu, F. B., \& Grodstein, F. (2005). Plasma C peptide level and cognitive function among older women without diabetes mellitus. Archives of Internal Medicine, 165, 1651-1656

Ott, A., Breteler, M. M., van Harskamp, F., Claus, J. J., van der Cammen, T. J., Grobbee, D. E., \& Hofman, A. (1995). Prevalence of Alzheimer's disease and vascular dementia: association with education. The Rotterdam study. British Medical Journal, 310, 970-973.

Panza, F., Capurso, C., D’Introno, A., Colacicco, A. M., Zenzola, A., Menga, R.,...Solfrizzi, V. (2008). Italian Longitudinal Study on Aging. Impact of depressive symptoms on the rate of progression to dementia in patients affected 
by mild cognitive impairment. International Journal Geriatric Psychiatry, 23, 726-734.

Panza, F., D’Introno, A., Colacicco, A. M., Capurso, C., Del Parigi, A., Caselli, R. J.,...Solfrizzi, V. (2005). Current epidemiology of mild cognitive impairment and other predementia syndromes. American Journal of Geriatric Psychiatry, 13, $633-644$.

Patterson, K., Graham, N., \& Hodges, J. R. (1994). Reading in dementia of the Alzheimer type: a preserved ability? Neuropsychology, 8, 395-407.

Paykel, E. S., Brayne, C., Huppert, F. A., Gill. C., Barkley, C., Gehlhaar, E.,...O’Connor, D. (1994). Incidence of dementia in a population older than 75 years in the United Kingdom. Archives of General Psychiatry, 51, 325-332.

Petersen, R. C., Smith, G. E., Waring, S. C., Ivnik, R. J., Tangalos, E. G. \& Kokmen, E. (1997). Aging, Memory, and Mild Cognitive Impairment. International Psychogeriatrics, 9, 65-69.

Petersen, R. C., Smith, G. E., Waring, S. C., Ivnik, R. J., Tangalos, E. G. \& Kokmen, E. (1999). Mild cognitive impairment: clinical characterization and outcome. Archives of Neurology, 56, 303-308.

Pfeffer, R. I., Kurosaki, T. T., Harrah, C. H., Jr., Chance, J. M., \& Filos, S. (1982). Measurement of functional activities in older adults in the community. Journal of Gerontology, 37, 323-329.

Plassman, B. L., Langa, K. M., Fisher, G. G., Heeringa, S. G., Weir, D. R., Ofstedal, M. B.,...Wallace, R. B. (2007). Prevalence of dementia in the United States: the aging, demographics, and memory study. Neuroepidemiology, 29, 125-132. 
Pratico, D., Clark, C. M., Lee, V. M., Trojanowski, J. Q., Rokach, J., \& FitzGerald, G. A. (2000). Increased 8,12-iso-iPF2alpha-VI in Alzheimer's disease: correlation of a noninvasive index of lipid peroxidation with disease severity. Annals of Neurology, 48, 809-812.

Pratico, D., Clark, C. M., Liun, F., Lee, V. Y., \& Trojanowski, J. Q. (2002). Increase of brain oxidative stress in mild cognitive impairment: a possible predictor of Alzheimer disease. Archives of Neurology, 59, 972-976.

Pratico, D., Uryu, K., Leight, S., Trojanoswki, J. Q., \& Lee, V. M. (2001). Increased lipid peroxidation precedes amyloid plaque formation in an animal model of Alzheimer amyloidosis. Journal of Neuroscience, 21, 4183-4187.

Pratico, D., V, M. Y. L., Trojanowski, J. Q., Rokach, J., \& Fitzgerald, G. A. (1998). Increased F2-isoprostanes in Alzheimer's disease: evidence for enhanced lipid peroxidation in vivo. Journal of the Federation of American Societies for Experimental Biology, 12, 1777-1783.

Prince, M. J., Bird, A. S., Blizard, R. A., \& Mann, A. H. (1996). Is the cognitive function of older patients affected by antihypertensive treatment? Results from 54 months of the Medical Research Council's trial of hypertension in older adults. British Medical Journal, 312, 801-805.

Ravaglia, G., Fort,i P., Montesi, F., Lucicesare, A., Pisacane, N., Rietti, E.,...Mecocci, P. (2008). Mild cognitive impairment: epidemiology and dementia risk in an elderly Italian population. Journal of the American Geriatric Society, 56, 51-58.

Reckess, G. (2003). Antecedent Biomarkers in Alzheimer's Disease: Uses, Limitations, and Future Directions for Research. St. Louis, Missouri: Washington University 
Alzheimer's Disease Research Center.

Reid, L. M., \& Maclullich, A. M. (2006). Subjective memory complaints and cognitive impairment in older people. Dementia and Geriatric Cognitive Disorders, 22, $471-485$.

Reisberg, B., Ferris, S. H., de Leon, M. J., \& Crook, T. (1982). The Global

Deterioration Scale for assessment of primary degenerative dementia. American Journal of Psychiatry, 139, 1136-1139

Reitan, R. M. (1958). Validity of the trail making test as an indicator of organic brain damage. Perceptual Motor Skills, 8, 271-276.

Rey, A. (1964). L'examen clinique en psychologie. Paris: Presses Universitaires de France.

Risacher, S. L., Saykin, A. J., West, J. D., Shen, L., Firpi, H. A., \& McDonald, B. C. (2009). Baseline MRI predictors of conversion from MCI to probable AD in the ADNI cohort. Current Alzheimer's Research, 6, 347-361.

Rocca, W. A., Cha, R. H., Waring, S. C., \& Kokmen, E. (1998). Incidence of dementia and Alzheimer's disease: a reanalysis of data from Rochester, Minnesota, 19751984. American Journal of Epidemiology, 148, 51-62.

Rosen, W. G., Mohs, R. C., \& Davis, K. L. (1984). A new rating scale for Alzheimer's disease. American Journal of Psychiatry, 141, 1356-1364.

Ruitenberg, A., Ott, A., Swieten, J. C., Hofman, A., \& Breteler, M. M. B. (2001). Incidence of dementia: does gender make a difference? Neurobiology of Aging, $22,575-580$.

Schuff, N. \& Zhu, X. P. (2007). Imaging of mild cognitive impairment and early 
dementia. British Journal of Radiology, 80, 109-114.

Seeman, T. E., Huang, M. H., Bretsky, P., Crimmins, E., Launer, L., \& Guralnik, J. M. (2005). Education and APOE-e4 in longitudinal cognitive decline: MacArthur Studies of Successful Aging. Journals of Gerontology Series B: Psychological Sciences and Social Sciences, 60, 74-83.

Shah, A.K., \& Oppenheimer, D.M. (2008). Heuristics made easy: An effort-reduction framework. Psychological Bulletin, 134, 207-222.

Shepherd, J., Blauw, G. J., Murphy, M. B, Bollen, E. L., Buckley, B. M., Cobbe, S. M.,...Westendorp, R. G., for the PROSPER Study Group (Prospective Study of Pravastatin in the Elderly at Risk). (2002). Pravastatin in elderly individuals at risk of vascular disease (PROSPER): A randomised controlled trial. Lancet, 360, $1623-1630$.

Slooter, A. J., Cruts, M., Hofman, A., Koudstaal, P. J., van der Kuip, D., de Ridder, M. A.,...van Duijn, C. M. (2004). The impactof APOE on myocardial infraction, stroke, and dementia: The Rotterdam study. Neurology, 62, 1196-1198.

Solfrizzi, V., Panza, F., Colacicco, A. M., D’Introno, A., Capurso, C., Torres, F.,... Capurso, A., Italian Longitudinal Study on Aging Working Group. (2004). Vascular risk factors, incidence of MCI, and rates of progression to dementia. Neurology, 63, 1882-1891.

Stern, Y., Gurland, B., Tatemichi, T. K., Tang, M. X., Wilder, D., \& Mayeux, R. (1994). Influence of education and occupation of the incidence of Alzheimer's disease. Journal of the American Medical Association, 271, 1004-1010.

Tan, Z. S., Seshadri, S., Beiser, A., Wilson, P. W., Kiel, D. P., Tocco, M.,...Wolf, P. A. 
(2003). Plasma total cholesterol level as a risk factor for Alzheimer disease: The Framingham Study. Archives of Internal Medicine, 163, 1053-1057.

Tabert, M. H., Manly, J. J., Liu, X., Pelton, G. H., Rosenblum, S.,...Devanand, D. P. (2006). Neuropsychological prediction of conversion to Alzheimer disease in patients with mild cognitive impairment. Archives of General Psychiatry, 63, 916924.

Teng, E., Becker, B. W., Woo, E., Knopman, D. S., Cummings, J. L., \& Lu, P. H. Utility of the Functional Activities Questionnaire for Distinguishing Mild Cognitive Impairment From Very Mild Alzheimer's Disease.

Tervo, S., Kivipelto, M., Hanninen, T., Vanhanen, M., Hallikainen, M., Mannermaa, A., \& Soininen, H. (2004). Incidence and risk factors for mild cognitive impairment: a population based three-year follow-up study of cognitively healthy elderly subjects. Dementia and Geriatric Cognitive Disorders, 17, 196-203.

Thongboonkerd, V. (2007). Practical points in urinary proteomics. Journal of Proteome Research, 6, 3881-3890.

Tierney, M. C., Szalai, J., Dunn, E., Geslani, D., \& McDowell, I. (2000). Prediction of Probable Alzheimer's disease in patients with symptoms suggestive of memory impairment: value of the Mini-Mental State Examination. Archives of Family Medicine, 9, 527-532.

Tierney, M. C., Szalai, J. P., Snow, W. G., Fisher, R. H., Nores, A., Nadon, G.,...St George-Hyslop, P. H. (1996). Prediction of probable Alzheimer's disease in memory impaired patients: a prospective longitudinal study. Neurology, 46, 661665. 
Tombaugh, T. M., \& McIntyre, N. J. (1992). The Mini-Mental State Examination: A comprehensive review. Journal of the American Geriatrics Society, 40, 922-935.

Tversky, A. \& Kahneman, D. (1974). Judgment under Uncertainty: Heuristics and Biases. Science, 185, 1124-1131.

Tzourio, C., Anderson, C., Chapman, N., Woodward, M., Neal, B., MacMahon, S., \& Chalmers, J., for the PROGRESS Collaborative Group. (2003). Effects of blood pressure lowering with perindopril and indapamide therapy on dementia and cognitive decline in patients with cerebrovascular disease. Archives of Internal Medicine, 163, 1069-1075.

Valenzuela, M. J. (2008). Brain reserve and the prevention of dementia. Current Opinion in Psychiatry, 21, 296-302.

Valenzuela, M. J. \& Sachdev, P. (2006). Brain reserve and cognitive decline: a nonparametric systematic review. Psychological Medicine, 36, 1065-1073.

Verghese, J., LeValley, A., Derby, C., Kuslansky, G., Katz, M., Hall, C.,...Lipton, R. B. (2006). Leisure activities and the risk of amnestic mild cognitive impairment in the elderly. Neurology, 66, 821-827.

Wedding, D., \& Faust, D. (1989). Clinical judgment and decision making in neuropsychology. Archives of Clinical Neuropsychology, 4, 233-265.

Whitwell, J. L., Shiung, M. M., Przybelski, S. A., Weigand, S. D., Knopman, D. S., Boeve, B. F.,...Jack, C. R. Jr. (2008). MRI patterns of atrophy associated with progression to AD in amnestic mild cognitive impairment. Neurology, 70, 512520.

Winblad, B., Palmer, K., Kivipelto, M., Jelic, V. Fratiglioni, L., Wahlund, L.- 
O.,...Petersen, R. C. (2004). Mild cognitive impairment - Beyond controversies, towards a concensus: report of the International Working Group on Mild Cognitive Impairment. Journal of International Medicine, 256, 240-246.

Wolozin, B., Kellman, W., Ruosseau, P., Celesia, G. G., \& Siegel, G. (2000). Decreased prevalence of Alzheimer's disease associated with 3-hydroxy-3-methylglutaryl coenzyme A reductase inhibitors. Archives of Neurology, 57, 1439-1443.

World Health Organization: International Statistical Classification of Diseases and Related Health Problems, 10th Revision (ICD-10). Geneva, Switzerland: World Health Organization, 1992.

Wragg, R. E., \& Jeste, D. V. (1989). Overview of depression and psychosis in Alzheimer's disease. American Journal of Psychiatry, 146, 577-587.

Yaffe, K., Haan, M., Byers, A., Tangen, C., \& Kuller, L. (2000). Estrogen use, APOE, and cognitive decline: Evidence of gene environment interaction. Neurology, 54, 1949-1954.

Yao, Y., Zhukareva, V., Sung, S., Clark, C. M., Rokach, J., Lee, V. M. (2003). Enhanced brain levels of 8,12-iso-iPF(2alpha)-VI differentiate AD from frontotemporal dementia. Neurology, 61, 475-478.

Yesavage, J. A. \& Sheikh, J. I. (1986). Geriatric Depression Scale (GDS): Recent evidence and development of a shorter version. Clinical Gerontologist, 5, 165173.

Yoshitake, T., Kiyohara, Y., Kato, I., Ohmura, T., Iwamoto, H., Nakayama, K.,...Fujishima, M. (1995). Incidence and risk factors of vascular dementia and 
Alzheimer's disease in a defined elderly Japanese population: the Hisayama study. Neurology, 45, 1161-1168.

Zaudig, M. (1992). A new systematic method of measurement and diagnosis of "mild cognitive impairment" and dementia according to ICD-10 and DSM-III-R criteria. International Psychogeriatrics, 2, 203-219.

Zaudig, M., \& Hillner, W. (1991). SIDAM-Structured interview for the diagnosis of dementia of the Alzheimer type, multi-infarct dementia, and dementias of other etiology according to DSM-III-R and ICD-10. The SIDAM user's guide. Munich, Germany: Logomed.

Zaudig, M., Mittelhammer, J., Hiller, W., Pauls, A., Thora., Morinigo, A., \& Mombour, W. (1991). SIDAM-A structured interview for the diagnosis of dementia of the Alzheimer type, multi-infarct dementia, and dementias of other etiology according to DSM-III-R and ICD-10. Psychological Medicine, 21, 225-236. 


\section{Appendix A: Model and Equation Derived from Neurocognitive Performances}

The resulting equation constructed from neurocognitive performance is as follows $($ see Table 9): Predicted Score $=($ Constant $[-0.073])+($ Logical Memory $\times$ $-0.177)+($ Clock Drawing $\times-0.273)+($ AVLT Trial $3 \times-0.205)+$ $(A V L T$ Trial 5 Intrusions $\times 0.203)+($ AVLT Recognition z score $\times-0.339)+$ $($ AVLT Recognition Errors $\times 0.112)+($ Digit Span z score $\times 0.219)+$ $($ Animal Naming $\times-0.064)+($ Animal Naming Perseverations $\times 0.176)+$ (Trails $A \times 0.014)+($ BNT Stimulus Cues $\times-0.109)$.

Examples may be useful for those not familiar with regression-based models (see Table 9). For this sample of people with MCI, the following means were observed: 4.45 on Logical Memory II, 4.20 on Clock Drawing, 6.34 on AVLT Trial 3, 5.46 on AVLT Total Intrusions for Trial 5, -0.82 for AVLT Recognition z-score, 2.08 for AVLT Recognition Errors, 0.41 for Digit Span z-score, 15.82 on Animal Naming, 1.12 on Animal Naming Total Perseverations, 43.93 on Trails A, and 2.65 on BNT Stimulus Cues. Using average neurocognitive performances and the constant obtained for this model (-0.073), this sample of people with MCI would have an overall score of -2.08 above the clinical cutoff of -2.32 . This means the sample as a whole is demonstrating an increased risk of conversion.

However, most applications of this model will likely occur at the individual level, and one such example can be found in Table 9. For this example (i.e., $-0.073-[0.177 * 0]$ $-[0.273 * 4]-[0.205 * 5]+[0.203 * 0]+[0.339 * 1.333]+[0.112 * 0]+[0.219 * 0.333]-$ $[0.064 * 16]+[0.176 * 3]+[0.014 * 39]-[0.109 * 4]=-2.078)$, the obtained score also lies above the clinical cutoff of -2.32 , so conversion within a 2-year period is likely. For a 
person who scored 8.0 on Logical Memory II, 5.0 on Clock Drawing, 7.0 on AVLT Trial 3, 2.0 on AVLT Total Intrusions for Trial 5, 0.09 for AVLT Recognition z-score, 1.0 for AVLT Recognition Errors, 0.41 for Digit Span z-score, 17.0 on Animal Naming, 1.0 on Animal Naming Total Perseverations, 34.0 on Trails A, and 2.0 on BNT Stimulus Cues the equation based on neuropsychological performance would be as follows: $-0.073-$ $[0.177 * 8]-[0.273 * 5]-[0.205 * 7]+[0.203 * 2]+[0.339 * 0.09]+[0.112 * 1]+$ $[0.219 * 0.41]-[0.064 * 17]+[0.176 * 1]+[0.014 * 34]-[0.109 * 2]=-4.3047$. This obtained scores lies well below the clinical cutoff of -2.32 , so conversion over a one-year period is unlikely. For those interested, an Excel spreadsheet and instructions for use can be obtained from the first author of this study that will calculate this equation. 


\section{Appendix B: Model and Equation Derived from Neuroimaging Data}

The resulting equation constructed from imaging variables is as follows (see Table 9):

Predicted Score $=$

(Constant [68.101] $)+($ Cumulative Temporal Lobe Atrophy $\times-0.063)+$ (Left Hippocampal Volume $\times-0.002)+($ Right Precentral Grey Matter $\times$ 19.218) + (Right Frontal Mid Orbital Grey Matter $\times-19.653)+$ $($ Left Frontal Mid Orbital Grey Matter $\times 12.989)+$ (Left Frontal Medial Orbital Grey Matter $\times 11.335)+$ (Right Posterior Cingulum Grey Matter $\times-15.869)+$ (Right Amygdala Grey Matter $\times-7.958) \times$ (Left Superior Parietal Grey Matter $\times-6.119) \times$ (Left Superior Temporal Grey Matter $\times-14.076)+$ (Right Cerebellum Grey Matter × 14.472).

Examples of this regression-based model are provided in Table 10. For this sample of people with MCI the following means were observed: cumulative temporal lobe atrophy of $983 \mathrm{~mm}^{3}$, left hippocampus volume of $1799 \mathrm{~mm}^{3}$, right precentral grey matter of 0.282 $\mathrm{mm}^{3}$, right frontal mid orbital grey matter of $0.432 \mathrm{~mm}^{3}$, left frontal inferior orbital grey matter of $0.397 \mathrm{~mm}^{3}$, left frontal medial orbital grey matter of $0.453 \mathrm{~mm}^{3}$, right posterior cingulum grey matter of $0.333 \mathrm{~mm}^{3}$, right amygdala grey matter of $0.62 \mathrm{~mm}^{3}$, left superior parietal grey matter of $0.30 \mathrm{~mm}^{3}$, left superior temporal grey matter of 0.432 $\mathrm{mm}^{3}$, right cerebellum grey matter of $0.48 \mathrm{~mm}^{3}$. Using these averages and the constant obtained for this model (68.101), this sample of people with MCI would have an overall 
score of -1.301 -above the clinical cutoff of -2.04 . This means the sample as a whole is demonstrating an increased risk of conversion. However, as with the neurocognitive model, most applications will likely occur at the individual level, and one such example can be found in Table 10. For this example (i.e., 68.101 - [0.063*991.279]$[0.002 * 1959.83]+[19.218 * 0.374]-[19.653 * 0.528]+[12.989 * 0.446]+[11.335 * 0.527]$ $-[15.869 * 0.366]-[7.958 * 0.741]-[6.119 * 0.387]-[14.076 * 0.450]+[14.472 * 0.591]=$ -1.519), the obtained score also lies above the clinical cutoff, so conversion within a 2year period is likely. For those interested, an Excel spreadsheet and instructions for use can be obtained from the first author of this study that will calculate this equation. 


\title{
CURRICULUM VITAE
}

\author{
Adam Gerstenecker, M.S. \\ 318 Oaks Dr. \\ Birmingham, AL 35209 \\ (606) 776-9973 \\ Atgers01@1ouisville.edu
}

\section{$\underline{\text { EDUCATION }}$}

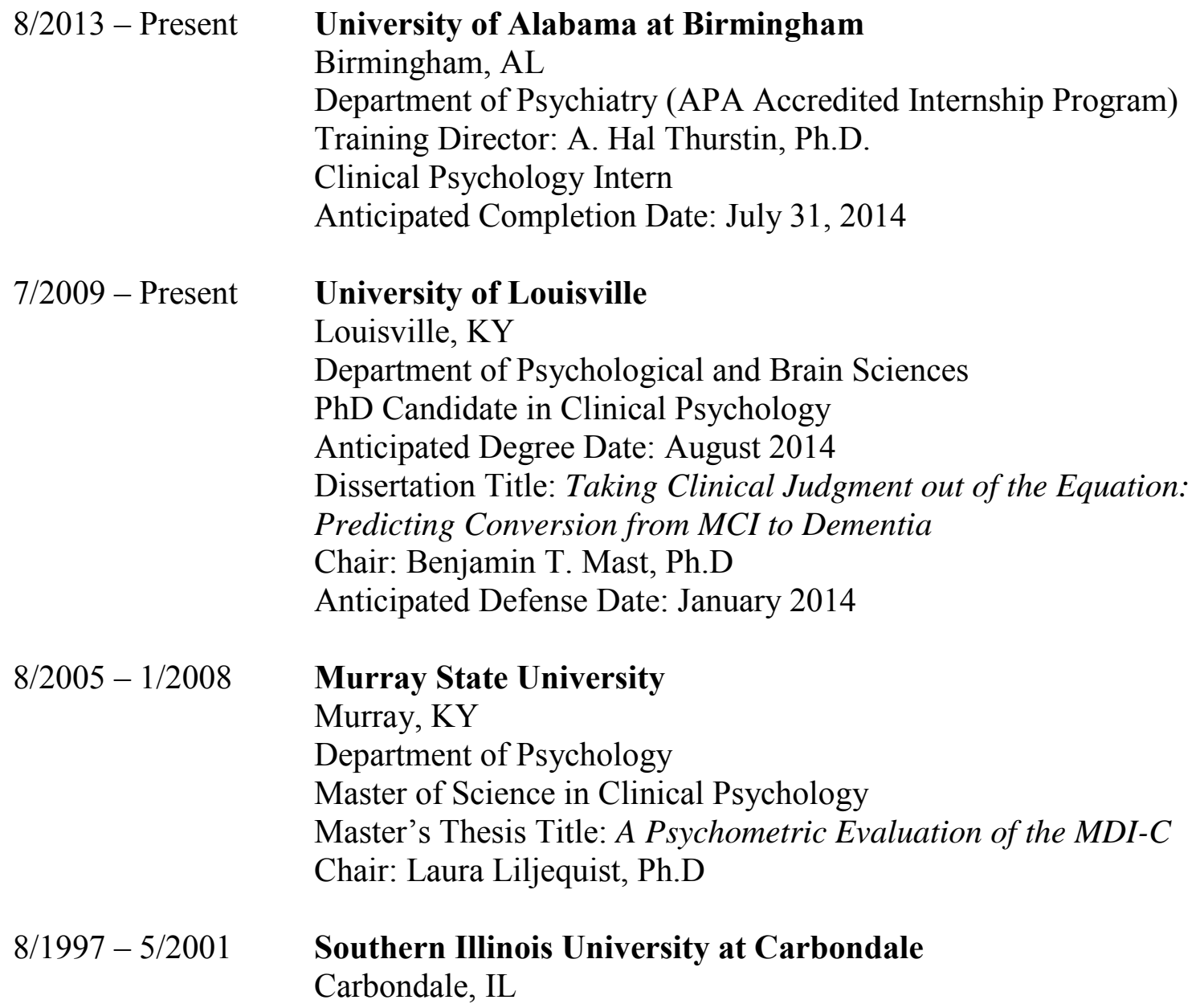


Bachelor of Science

Major: Psychology

Minor: Biology

Senior Project Title: Smoking Behavior and EEG Brainwave

Patterns

\section{CLINICAL EXPERIENCE}

\section{8/2013 - Present Clinical Psychology Intern, Adult Neuropsychology Emphasis University of Alabama at Birmingham Department of Psychiatry}

Training Director: A. Hal Thurstin, Ph.D.

Provide outpatient and inpatient neuropsychological and psychological services to patients representing a range of ethnic, socioeconomic, and educational backgrounds. Didactic experiences include neuropsychology clinical case conferences, intern seminars, brain cuttings, and various departmental grand rounds (i.e., neurology, psychiatry, physical medicine and rehabilitation). Past, present, and future rotations are listed below.

\section{8/2013 - 11/2013 UAB Center for Psychiatric Medicine - Neuropsychology} Service

Supervisors: A. Hal Thurstin, Ph.D., \& Michelle Benjamin, Ph.D. Conducted neurocognitive and mood assessments in a medical setting to aid in differential diagnosis and treatment planning for older adults. Conducted neurocognitive and mood assessments as part of the preoperative transplant recipient evaluation (i.e., heart, lung, and liver) and preoperative gastric bypass evaluation. Functioned as the living donor advocate for potential kidney donors.

\section{8/2013 - 11/2013 UAB Department of Neurology - Neuropsychology Division} Supervisors: Daniel Marson, J.D., Ph.D. \& Kristen Triebel, Psy.D. Evaluated the neuropsychological and psychiatric functioning of patients referred from a wide array of physicians but primarily UAB neurologists. Primary referrals were adults over the age of 50. Duties included an emphasis on report writing and, in particular, on forming diagnostic impressions and etiological considerations. Duties also included conducting neurocognitive and mood assessments as part of the preoperative DBS evaluation.

\section{2/2013 - 4/2014 Birmingham VA Medical Center - Neuropsychology Clinic Supervisor: Paul Blanton, Ph.D. \\ Evaluate the neuropsychological and psychiatric functioning of patients referred from a wide array of physicians but primarily Birmingham VA Medical Center neurologists. Primary referrals are older adult veterans. Duties include an emphasis on testing and report writing and, in}


particular, on forming diagnostic impressions and etiological considerations.

12/2013 - 4/2014 UAB Spain Rehabilitation Center - Neuropsychology Service Supervisor: Thomas Novack, Ph.D., ABPP-CN

Provide neuropsychological and psychological services to inpatients and outpatients with a history of TBI. Primary duties include: conducting neuropsychological, mood, and functional assessments; report writing; and providing consultation to other professionals.

4/2014 - 8/2014 UAB Community Psychiatry Program - Neuropsychology Service Supervisor: Robert Savage, Ph.D.

Will conduct neuropsychological services in inpatient and outpatient settings for patients with Serious Mental Illness (SMI) and other severe psychiatric disorders.

4/2014 - 8/2014 UAB Spain Rehabilitation Center - Neuropsychology Service Supervisor: Amy Knight, Ph.D.

Will provide neuropsychological and psychological services in an inpatient rehabilitation setting for patients with a wide range of medical conditions (e.g., orthopedic injuries, cardiovascular disease, amputations, peripheral vascular disease, end stage renal disease, dementia, cancer, and pressure ulcer flap surgery). Primary duties include: conducting neuropsychological, mood, and functional assessments; report writing; and providing consultation to other professionals.

8/2009 - 5/2013 The Noble H. Kelley Psychological Services Center University of Louisville Supervisors: Janet Woodruff-Borden, Ph.D., Benjamin Mast, Ph.D., Bernadette Walter, Ph.D., \& David Winsch, Ph.D.

Provided psychotherapy to adults with a wide range of presenting problems and diagnoses. Conducted a wide range of assessments with older adult, adult, and child populations (i.e., neurocognitive, learning disability, advanced placement, and ADHD).

\section{8/2009 - 8/2011 University of Louisville's Family and Geriatric Medicine} Center

Supervisor: Benjamin Mast, Ph.D.

Provided psychotherapy to older adults reporting significant symptoms of depression. Conducted mood and neuropsychological assessments in an older adult population.

8/2009 - 8/2011 University of Louisville's Geriatric Evaluation Team (GET) Supervisor: Benjamin Mast, Ph.D. 
Conducted neurocognitive and mood assessments in a medical setting to aid in the diagnosis and treatment planning for older adults. Worked with GET team members (i.e., geriatrician, geriatric fellow, gerontologist, and geriatric pharmacist) to assess, diagnose, and develop treatment strategies for older adults.

\section{7/2007 - 7/2009 NeuroRestorative: Brain Injury Recovery \& Spinal Cord Rehabilitation \\ 927 Tipton Lane \\ Paducah, KY 42003 \\ Worked as a Behavior Specialist/Therapist at an inpatient, post-acute, residential facility for people with acquired brain injuries. Provided psychological services including psychological assessments and functional analyses. Developed behavior support plans including implementation and training of direct care staff. Provided psychotherapy to residents and functioned as a group facilitator. Received individual supervision from a licensed psychologist (Laura Liljequist, PhD) in accordance with state stipulations for licensed psychological associates.}

\section{8/2004 - 5/2007 Murray State University Psychological Center} Supervisors: Laura Liljequist, Ph.D., Renee Duncan, Ph.D., \& Susan Rosenberger, Ph.D.

Provided psychotherapy to children and adults with a wide range of presenting problems and diagnoses. Conducted a wide range of assessments with older adult, adult, and child populations (i.e., neurocognitive, learning disability, advanced placement, and ADHD). Provided psychotherapy to families and couples.

\section{RESEARCH EXPERIENCE ON GRANT FUNDED PROJECTS}

Decisional Capacity in Patients with Metastatic Brain Cancer: KL2 Mentored Career Developmental Award grant KL2 TR000166, \$296,354 Research Assistant; PI: K. Triebel

August 2013 - Present

Trained a doctoral level research assistant on data collection and manuscript preparation. Analyzed data for trends. Lead author on multiple manuscripts. Contributing author on other manuscripts.

Genetic and Environmental Risk Factors for Progressive Supranuclear Palsy:

National Institutes of Health grant 5R01AG024040-04, \$2,723,352.

Research Assistant; PI: I. Litvan

August 2009 - July 2013

Provided quality control of data collected from 13 sites. Trained psychometrists at multiple sites. Analyzed neuropsychological and neuropsychiatric data for trends. Lead author on multiple manuscripts. Contributing author on other manuscripts. 
Predicting outcome in Parkinson's disease cognitive impairment:

Canadian Institutes of Health research grant, $\$ 183,364$

Collaborator; PI: S. Duff-Canning and C. Marras

January 2013 - July 2013

Oversaw all components of the neuropsychological aspects conducted at the University of Louisville (including quality control, administration of measures, recruitment and scheduling of participants, coordination of testing, and supervision and training of psychometrists).

Validating the Montreal Cognitive Assessment (MoCA) for the Diagnosis of Mild Cognitive Impairment in Parkinson's disease:

Grant provided through the Michael J. Fox Foundation for Parkinson's Research Research Assistant; PI: S. Duff-Canning and C. Marras

August 2010 - January 2013

Oversaw all components of the neuropsychological aspects conducted at the University of Louisville (including quality control, administration of measures, recruitment and scheduling of participants, coordination of testing, and supervision and training of psychometrists). Contributing author on one manuscript.

\section{OTHER RESEARCH EXPERIENCE}

Taking Clinical Judgment out of the Equation: Predicting Conversion from MCI to Dementia Dissertation - University of Louisville

Chair: Dr. Benjamin Mast

March 2012 - Present

Examining rates of conversion from mild cognitive impairment to dementia (primarily Alzheimer's disease). Using demographics, neuropsychological testing, biomarkers, imaging, activities of daily living, and emotional/behavioral functioning to construct a statistical equation designed to predict conversion from MCI to dementia.

Research Assistant-University of Louisville Aging and Neuropsychology Lab August 2009 - July 2013

Administered neuropsychological test batteries to older adults. Analyzed data to determine patterns of performance of healthy older adults, older adults with mild cognitive impairment, and older adults with various types of dementia syndromes. Lead author on one manuscript.

Study of Thinking and Relationships (STAR)

University of Louisville

PI: Dr. Sarah Rowe

August 2010 - August 2012

Administered neuropsychological assessments to older adults. Trained undergraduate team members on test battery and supervised undergraduate team members as they conducted duties. 
A Psychometric Evaluation of the MDI-C

Master's Thesis - Murray State University

Chair: Dr. Laura Liljequist

August 2005 - May 2007

Examined the psychometric properties of the Multiscore Depression Inventory for Children in a clinical sample.

Research Assistant - Southern Illinois University, Integrated Neuroscience Laboratory PI: Dr. David Gilbert

August 2000 - May 2001

Recruited and interviewed participants, obtained social and medical histories, administered the instruments utilized in the study (including an electroencephalogram), and collected and entered data.

\section{PUBLICATIONS}

Gerstenecker, A., L., Duff, K., Marson, D. C., Meneses, K., Fiveash, J., Cutter, G., Meyers, C. A., Watts, O., Martin, R. C., Nabors, L. B., \& Triebel, K. L. (in review). Cognitive Predictors of Understanding in a Medical Context in Patients with Newly Diagnosed Brain Metastases.

Triebel, K. L., Duff, K., Gerstenecker, A., Marson, D. C., Meneses, K., Fiveash, J., Cutter, G., Meyers, C. A., Watts, O., Martin, R. C., \& Nabors, L. B. (in review). Cognitive Predictors of Reasoning in a Medical Context in Patients with Newly Diagnosed Brain Metastases.

Gerstenecker, A., Marson, D. C., Meneses, K., Fiveash, J., Cutter, G., Meyers, C. A., Watts, O., Martin, R. C., Nabors, L. B., \& Triebel, K. L., (in review). Cognition in Patients with Newly Diagnosed Brain Metastasis.

Triebel, K. L., Gerstenecker, A., Marson, D. C., Meneses, K., Fiveash, J., Cutter, G., Meyers, C. A., Watts, O., Martin, R. C., \& Nabors, L. B. (in review). Medical Decision-Making Capacity of Individuals with Newly Diagnosed Brain Metastases: A Pilot Study.

Ludwin, B., Gerstenecker, A., Factor, P., Looney, S., \& Meeks, S. (in review). Affect variability and selective serotonin reuptake inhibitor use in long-term care residents with major depressive disorder.

Gerstenecker, A., Mast, B., Shah, S., \& Meeks, S. (in press). Tracking the cognition of nursing home residents. Clinical Gerontologist.

Duff, K., Shprecher, D., Litvan, I., Gerstenecker, A., \& Mast, B. (2013). Correcting for demographic variables on the modified Telephone Interview for Cognitive Status. American Journal of Geriatric Psychiatry. 10.1016/j.jagp.2013.08.007 
Gerstenecker, A., Duff, K., Mast, B., \& Litvan, I., for the ENGENE-PSP Study Group. (2013). Behavioral Abnormalities in Progressive Supranuclear Palsy. Psychiatry Research, 210, 1205-1210.

Gerstenecker, A., Mast, B., \& Litvan, I. (2013). Neuropsychiatric and cognitive disorders in other parkinsonian disorders. In D. Aarsland, J. Cummings, D. Weintraub, \& R. Chaudhuri (Eds.), Neuropsychiatric and Cognitive Changes in Parkinson's Disease and Related Movement Disorders (261-274). Cambridge, United Kingdom: Cambridge University Press.

Duff, K., Gerstenecker, A., \& Litvan, I., for the ENGENE-PSP Study Group. (2013). Functional Impairment in Progressive Supranuclear Palsy. Neurology, 80, 380384.

Gerstenecker, A., Mast, B., Duff, K., Ferman, T. J., \& Litvan, I., for the ENGENE-PSP Study Group. (2013). Executive Dysfunction is the Primary Cognitive Impairment in Progressive Supranuclear Palsy. Archives of Clinical Neuropsychology, 28, 104-113.

Marras, C., Armstrong, M. J., Meaney, C. A., Fox, S., Rothberg, B. Reginold, W. TangWai, D. $\quad$ F., Gill, D., Eslinger, P. J., Zadikoff, C., Kennedy, N., Marshall, F. J., Mapstone, M., Chou, K. L., Persad, C., Litvan, I., Mast, B. T., Gerstenecker, A. T., Weintraub, S., Duff-Canning, S. (2013). Measuring Mild Cognitive Impairment in Patients with Parkinson's disease. Movement Disorders, 28, 626633.

Gerstenecker, A. \& Mast, B. (2012). Aging, Rehabilitation, and Psychology. In P. Kennedy (Ed.), The Oxford Handbook of Rehabilitation Psychology (189-210). New York, NY: Oxford Press.

Mast, B. \& Gerstenecker, A. (2010). Screening Instruments and Brief Batteries for Dementia. In P. Lichtenberg (Ed.), Handbook of Assessment in Clinical Gerontology (503-530), New York, NY: John Wiley \& Sons, Inc.

\section{POSTER PRESENTATIONS}

Gerstenecker, A. \& Mast, B. (2014). Constructing an equation to predict conversion from mild cognitive impairment to dementia. To be Presented at the International Neuropsychological Society, Seattle, WA.

Gerstenecker, A., Mast, B., \& Litvan, I. (2012). Neuropsychiatric features in progressive supranuclear palsy. Presented at the Annual Meeting of the National Academy of Neuropsychology, Nashville, TN.

Gerstenecker, A., Mast, B., \& Litvan, I. (2011). Are Progressive Supranuclear Palsy 
Cognitive Deficits Mostly Frontal? Presented at the Annual Meeting of the International Neuropsychological Society, Boston, MA.

Gerstenecker A., Shah, S., Mast, B., \& Meeks, S. (2011). Tracking the Performance of Nursing Home Residents on the MMSE: Can Variability be Used to Predict a Dementia Diagnosis? Presented at the Annual Meeting of the Gerontological Society of America, Boston, MA.

Gerstenecker, A. \& Liljequist, L. (2008). A Psychometric Evaluation of the Multi-score Depression Inventory for Children. Presented at the annual meeting of the American Psychological Association, Boston, MA.

\section{SUPERVISION EXPERIENCE}

$\begin{array}{ll}\text { 8/2009- 7/2013 } & \text { The Noble H. Kelley Psychological Services Center } \\ & \text { University of Louisville } \\ & \text { Provided peer-supervision to PhD level clinical psychology } \\ & \text { students in the areas of psychotherapy, assessment, and case } \\ & \text { management. }\end{array}$

8/2009 - 8/2011 University of Louisville's Family and Geriatric Medicine Center

Provided peer-supervision to $\mathrm{PhD}$ level clinical psychology students with older adult clients in the areas of psychotherapy, assessment, and case management.

8/2009 - 8/2011 University of Louisville's Geriatric Evaluation Team (GET) Provided peer-supervision to $\mathrm{PhD}$ level clinical psychology students in the areas of assessment, differential diagnoses, and providing consultation to an interdisciplinary team.

\section{1/2008 - 7/2009 NeuroRestorative: Brain Injury Recovery \& Spinal Cord Rehabilitation}

Worked in a supervisory capacity as a full-time Behavior Specialist/Therapist at an inpatient, post-acute, residential facility for people with an acquired brain injury. Provided supervision to a wide range of professionals (i.e., occupational therapists, psychotherapists, nurses, speech language pathologists, and case workers) as well as direct care staff regarding the implementation of behavior support plans.

\section{1/2004 - 5/2007 Murray State University Psychological Center} Provided peer-supervision to masters level clinical psychology students in the areas of psychotherapy, assessment, and case management. 


\section{TEACHING EXPERIENCE}

Guest lecturer. Presented neuropsychology module for students enrolled in an auditory science doctoral program (Fall 2013).

Graduate Teaching Assistant (TA) for Psychology 201: Introduction to Psychology (Fall 2012).

Graduate Teaching Assistant (TA) for Psychology 582: Introduction to Clinical

Psychology (Summer 2011).

Guest lecturer. Presented neuropsychology module for students enrolled in an auditory science doctoral program (Fall 2011).

Graduate Teaching Assistant (TA) for Psychology 582: Introduction to Clinical Psychology (Summer 2010).

\section{SERVICE}

Invited peer reviewer for Psychiatry Research

Invited peer reviewer for Neuropsychiatry

Invited peer reviewer for BRAIN

Louisville Chapter's Multiple Sclerosis Family Weekend (November, 2011)

Louisville Zoo - Louisville, KY

Facilitated group discussion for people diagnosed with Multiple Sclerosis.

West Louisville Health Fair (September, 2011)

Westend Baptist Church - Louisville, KY

Educated community members about services offered by the University of Louisville's Psychological Services Center.

National Memory Screening Day - Louisville, KY (November, 2010)

University of Louisville

Conducted dementia screenings and offered referral services.

Sexual Assault Awareness Seminar - Murray, KY (April, 2007)

Murray State University

Provided crisis intervention and referrals for students following a seminar on sexual assault.

Eating Disorder Awareness - Murray, KY (March, 2006)

Body Fair, Murray State University

Conducted eating disorder screenings and offered referral services. 


\section{AWARDS AND HONORS RECEIVED}

Research Assistantship, University of Louisville

One of five finalists for the Association of Test Publishers (ATP) Award for Best Student Poster Focused on Assessment and Western Psychological Services' Emerging Leaders in Assessment Travel Award presented at the 2008 APA Conference in Boston, MA.

Graduate Assistantship, Murray State University (2005-2007)

\section{PROFESSIONAL ASSOCIATIONS}

Gerontological Society of America

National Academy of Neuropsychology

International Neuropsychological Society

\section{REFERENCES}

BENJAMIN MAST, PhD

Associate Professor

Vice Chair Psychological and Brain Sciences

University of Louisville

Life Sciences Room 317

Louisville, KY 40292

Phone: (502) 852-3280

b.mast@louisville.edu

JANET WOODRUFF-BORDEN, PhD

Professor

Director of Clinical Training

University of Louisville

Department of Psychological and Brain Sciences

Life Sciences Room 357

Louisville, KY 40292

Phone: (502) 852-6070

j.woodruff-borden@louisville.edu

KRISTEN TRIEBEL, PsyD

Assistant Professor

University of Alabama at Birmingham

Department of Neurology, Division of Neuropsychology

650 Sparks Center

Birmingham, AL 35294

Phone: (205) 934-1272 
ktriebel@uab.edu
A. HAL THURSTIN, PhD
Professor
Chief Psychologist, Internship Training Director
University of Alabama at Birmingham
Department of Psychiatry
472 Center for Psychiatric Medicine
Phone: (205) 934-1518
hadrian@uab.edu 